\title{
Lagrangian blow-ups, blow-downs, and applications to real packing
}

\author{
Antonio Rieser
}

Copyright (C) 2014 by Antonio Rieser. All rights reserved.

Given a symplectic manifold $(M, \omega)$ and a Lagrangian submanifold $L$, we construct versions of the symplectic blow-up and blow-down which are defined relative to $L$. We further show that if $M$ admits an anti-symplectic involution $\phi$, i.e., a diffeomorphism such that $\phi^{2}=$ Id and $\phi^{*} \omega=-\omega$, and we blow-up an appropriately symmetric embedding of symplectic balls, then there exists an antisymplectic involution on the blow-up $\tilde{M}$ as well. We then derive a homological condition for real Lagrangian surfaces $L=\operatorname{Fix}(\phi)$ which determines when the topology of $L$ changes after a blowdown, and we use these constructions to study the relative packing numbers and packing stability for real symplectic four manifolds which are non-Seiberg-Witten simple.

1.1 Setting and notation
1.2 Anti-symplectic involutions and almost complex structures

2 Constructing the relative and real blow-up and blow-down

2.2 Blow-down

2.3 Invariant symplectic neighborhoods and the Moser stability theorem in real symplectic manifolds 


\section{Introduction}

The blow-up and blow-down constructions are important techniques in complex geometry, leading to methods for resolving singularities as well as classification schemes based on birational equivalence. In the symplectic category, the notion of blowing up a point or submanifold has also been defined and studied from various points of view, as in papers by Guillemin and Sternberg [11], Lerman [16], and McDuff and Polterovich [20]. When combined with the theory of $J$-holomorphic curves, the blow-up and blow-down have yielded a great deal of information on symplectic manifolds, notably in packing problems $[3,20]$, in the classification of rational and ruled symplectic 4-manifolds [13, 14, 17], and in the study of the topology of the space of symplectic embeddings of balls, as, for example, in [1, 15, 25]. In this note, we study relative and real versions of the symplectic blow-up and blow-down, in order to apply them to questions regarding the topology of Lagrangian submanifolds. The relative blow-up takes the pair $(M, L)$ and a set of relative ball embeddings $\psi: \coprod_{j=1}^{k}\left(B_{j}^{2 n}(1+2 \epsilon), \lambda_{j}^{2} \omega_{0}, B_{\mathbb{R}, j}(1+2 \epsilon)\right) \rightarrow(M, \omega, L)$ and obtains another pair $(M, \tilde{L})$, and a symplectic form $\tilde{\omega}$, in which the balls have been replaced by copies of the tautological disk bundle over $\mathbb{C} P^{n-1}$, and $\tilde{L}$ is Lagrangian in $(\tilde{M}, \tilde{\omega})$. The blow-down is the reverse procedure. The real blow-up and blow-down are similar constructions which also respect a so-called real structure on the manifolds.

As a first application, we study the packing problem in real symplectic manifolds. The relative and mixed packing problems were first introduced by Barraud and Cornea in [2], and upper bounds for the relative embedding of one ball on the Clifford torus in $\mathbb{C} P^{n}$ was given by Biran and Cornea in [4] 
using Pearl Homology. Buhovsky [6] further showed that the upper bound given for the Clifford torus is sharp. Schlenk, in [27], directly constructed relative packings of $k \leq 6$ balls in $\left(\mathbb{C} P^{2}, \mathbb{R} P^{2}\right)$ through a detailed analysis of the moment map. A related construction for packing $\mathbb{C} P^{2}$ for $k=7,8$ balls was done by Wieck in [29]. It is not immediately clear if Wieck's techniques can be made adapted to the relative setting, since the symplectic tunnelling technique that he introduces does not produce relative embeddings. In Section 4 , we construct relative embeddings using $J$-holomorphic techniques, following the general line of argument in $[3,20]$. Our results extend those of McDuff and Polterovich [20] and Biran [3] to the real setting. Our packing method depends on the presence of a real structure $\phi$ for which $L=\operatorname{Fix}(\phi)$, and because of this, we do not recover the lower bounds on the Clifford Torus considered by Buhovsky [6].

Many of the results in this paper first appeared in my Ph.D. thesis, carried out at the Université de Montréal under the supervision of Octav Cornea and François Lalonde.

\subsection{Setting and notation}

We now give several definitions and set notation for all that follows.

Definition 1.1. Let $\left(M^{2 n}, \omega\right)$ be a symplectic manifold. We say that a submanifold $L$ is Lagrangian if $\operatorname{dim} L=n$ and $\left.\omega\right|_{T L}=0$.

Definition 1.2. (1) We let $\mathcal{L}^{n}$ denote the tautological complex line bundle over $\mathbb{C} P^{n-1}$, and let $\mathcal{R}^{n}$ be the real tautological line bundle over $\mathbb{R} P^{n-1}$, i.e., $\mathcal{L}^{n}=\left\{(z, l) \in \mathbb{C}^{n} \times \mathbb{C} P^{n-1} \mid z \in l\right\}$ and $\mathcal{R}^{n}=\{(x, l) \in$ $\left.\mathbb{R}^{n} \times \mathbb{R} P^{n-1} \mid x \in l\right\}$. We will suppress the dimension $n$ when it is clear from the context.

(2) $\pi: \mathcal{L} \rightarrow \mathbb{C}^{n}$ and $\theta: \mathcal{L} \rightarrow \mathbb{C} P^{n-1}$ denote the canonical projections.

(3) $\mathcal{L}(r)$ and $\mathcal{R}(r)$ denote the canonical open disk bundles over $\mathbb{C} P^{n-1}$ and $\mathbb{R} P^{n-1}$, respectively, of radius $r$. Abusing notation, we will use $\mathcal{L}(0)$ and $\mathcal{R}(0)$ to refer to the zero section of these bundles.

(4) For each $\kappa, \lambda>0$, we define a closed two-form $\rho(\kappa, \lambda)$ on $\mathcal{L}(r)$ by

$$
\rho(\kappa, \lambda)=\kappa^{2} \pi^{*} \omega_{0}+\lambda^{2} \theta^{*} \sigma
$$

where $\omega_{0}$ is the standard form on $\mathbb{C}^{n}$, and $\sigma$ is the standard Kähler form on $\mathbb{C} P^{n-1}$, normalized so that $\int_{\mathbb{C} P^{1}} \sigma=\pi$. 
(5) Let $\tilde{c}: \mathcal{L} \rightarrow \mathcal{L}$ be the map $\tilde{c}(z, l)=(\bar{z}, \bar{l})$, i.e., the restriction to $\mathcal{L}$ of the complex conjugation map on $\mathbb{C}^{n} \times \mathbb{C} P^{n-1}$.

In addition, the manifolds we treat in our applications will have an additional structure, as defined by

Definition 1.3. Let $(M, \omega)$ be a symplectic manifold. A symplectic antiinvolution, or real structure, is a diffeomorphism $\phi: M \rightarrow M$ such that $\phi^{2}=$ Id and $\phi^{*} \omega=-\omega$. We call a symplectic manifold equipped with a real structure a real symplectic manifold, or simply a real manifold, if the symplectic form is understood.

Remark 1.4. Note that $\operatorname{Fix}(\phi)$ is Lagrangian.

Definition 1.5. Let $(M, \omega, \phi)$ and $\left(M^{\prime}, \omega^{\prime}, \phi^{\prime}\right)$ be real symplectic manifolds. We say that an embedding $\psi:\left(M^{\prime}, \omega^{\prime}, \phi^{\prime}\right) \rightarrow(M, \omega, \phi)$ is a real symplectic embedding if $\phi \circ \psi=\psi \circ \phi^{\prime}$ and $\psi^{*} \omega=\omega^{\prime}$.

Lemma 1.6. Let $\left(M, \omega_{0}\right)$ be a symplectic manifold, and let $\left(N, \omega_{1}, \phi\right)$ be a real symplectic manifold with symplectic form $\omega_{1}$ and real structure $\phi$. Suppose that there exists a symplectic embedding $\psi:\left(M, \omega_{0}\right) \rightarrow\left(N, \omega_{1}\right)$ such that $\operatorname{Im}(\phi \circ \psi)=\operatorname{Im}(\psi)$. Then there exists an anti-symplectic involution $c$ on $M$ such that $\phi \circ \psi=\psi \circ c$.

Proof. Define $c:=\psi^{-1} \circ \phi \circ \psi$. Then $\phi \circ \psi=\psi \circ c$ and $c^{*} \omega_{0}=\psi^{*} \phi^{*}\left(\psi^{-1}\right)^{*}$ $\psi^{*} \omega_{1}=-\omega_{0}$, so $\phi$ is an anti-symplectic involution on $M$.

With the notation in Definition 1.2, we have

Corollary 1.7. $\tilde{c}^{*} \rho(\kappa, \lambda)=-\rho(\kappa, \lambda)$, and $\mathcal{R}=\operatorname{Fix}(\tilde{c})$.

Proof. Let $c: \mathbb{C}^{n} \rightarrow \mathbb{C}^{n}$ and $\bar{c}: \mathbb{C} P^{n-1} \rightarrow \mathbb{C} P^{n-1}$ denote complex conjugation on $\mathbb{C}^{n}$ and $\mathbb{C} P^{n-1}$, respectively. Then by the definition of $\tilde{c}, \tilde{c}(z, l)=$ $(c(x), \bar{c}(l))$. Since $\mathbb{R}^{n}=\operatorname{Fix}(c)$ and $\mathbb{R} P^{n-1}=\operatorname{Fix}(\bar{c}), \mathcal{R}=\operatorname{Fix}(\tilde{c})$.

Now let $\left(v_{0}, w_{0}\right),\left(v_{1}, w_{1}\right) \in T_{(z, l)} \mathcal{L} \subset T_{z} \mathbb{C}^{n} \oplus T_{l} \mathbb{C} P^{n-1}$. Then

$$
\begin{aligned}
\tilde{c}^{*} \rho(\kappa, \lambda)\left(\left(v_{0}, w_{0}\right),\left(v_{1}, w_{1}\right)\right)= & \tilde{c}^{*} \pi^{*} \kappa^{2} \omega_{0}\left(\left(v_{0}, w_{0}\right),\left(v_{1}, w_{1}\right)\right) \\
& +\tilde{c}^{*} \theta^{*} \lambda^{2} \sigma\left(\left(v_{0}, w_{0}\right),\left(v_{1}, w_{1}\right)\right) \\
= & -\kappa^{2} \omega_{0}\left(v_{0}, v_{1}\right)-\lambda^{2} \sigma\left(w_{0}, w_{1}\right) \\
= & -\rho(\kappa, \lambda)\left(\left(v_{0}, w_{0}\right),\left(v_{1}, w_{1}\right)\right),
\end{aligned}
$$

which completes the proof. 
In order to put a symplectic form on the blow-up of a manifold $M$, we will need to consider the relative embeddings of symplectic manifolds, defined below.

Definition 1.8. Let $(M, \omega, L)$ and $\left(M^{\prime}, \omega^{\prime}, L^{\prime}\right)$ be symplectic manifolds with Lagrangians $L$ and $L^{\prime}$, respectively. We say that a map $\psi:\left(M^{\prime}, \omega^{\prime}, L^{\prime}\right) \rightarrow$ $(M, \omega, L)$ is a relative symplectic embedding when $\psi$ is a symplectic embedding, $\psi^{*} \omega=\omega^{\prime}$, and $\psi^{-1}(L)=L^{\prime}$.

We will be primarily concerned with the following example.

Example 1.9. Let $\left(M^{2 n}, \omega, L\right)$ be a symplectic manifold with Lagrangian $L$. Let $\left(B(\lambda), \omega_{0}\right)$ be the ball of radius $\lambda$ in $\mathbb{C}^{n}$ with the standard symplectic structure $\omega_{0}$, and let $B_{\mathbb{R}}(\lambda)$ denote the ball of radius $\lambda$ in $\mathbb{R}^{n} \subset \mathbb{C}^{n}$. Then a symplectic embedding $\psi:\left(B^{2 n}(\lambda), \omega_{0}\right) \hookrightarrow\left(M^{2 n}, \omega\right)$ is a relative symplectic embedding iff $\psi^{-1}(L)=B_{\mathbb{R}}(\lambda)$.

Remark 1.10. Note that in Definition 1.8, we have $\psi^{-1}(L)=L^{\prime}$, and not $\psi\left(L^{\prime}\right) \subseteq L$. This is an important distinction, as shown by the following example. Let $C$ denote an embedding of $S^{1}$ into $\mathbb{C}^{1}$, and let

$\Lambda:=\left\{\lambda \in \mathbb{R} \mid \exists\right.$ a relative embedding $\left.\psi:\left(B^{2}(1), \lambda^{2} \omega_{0}, B_{\mathbb{R}}(1)\right) \hookrightarrow\left(\mathbb{C}^{1}, \omega_{0}, C\right)\right\}$.

Let $\Lambda_{\text {sup }}:=\sup \Lambda$. Then for any $\lambda \in \Lambda, \lambda^{2} \pi \leq 2 A$, where $A$ is the area inside $C \subset \mathbb{C}^{2}$. Therefore $\Lambda_{\text {sup }} \leq \sqrt{\frac{2 A}{\pi}}$. If, however, we only require that $\psi\left(B_{\mathbb{R}}(1)\right) \subseteq C$, then $\Lambda$ is not bounded above.

Definition 1.11. Let $\psi: \coprod_{i=1}^{k}\left(B_{i}(r), \omega_{0}, B_{\mathbb{R}, i}(r)\right) \hookrightarrow(M, \omega, L)$ be a symplectic embedding, and let $\psi_{i}:=\left.\psi\right|_{B_{i}}$. If $p$ of the $\psi_{i}$ 's are relative embeddings, and for the other $q=k-p$ of the $\psi_{i}$ 's, we have $\operatorname{Im}\left(\psi_{i}\right) \cap L=\emptyset$, then we call $\psi$ a $(p, q)$-mixed embedding.

\subsection{Anti-symplectic involutions and almost complex structures}

Our constructions will use auxiliary almost complex structures which satisfy certain additional properties. In this section, we give the necessary definitions and prove the existence of the complex structures that we need.

Definition 1.12. Let $(M, \omega)$ be a symplectic manifold. Then an almost complex structure $J$ tames $\omega$ or is $\omega$-tame if $\omega(\cdot, J \cdot)>0$. 
Definition 1.13. Let $(M, \omega)$ be a symplectic manifold. Then an almost complex structure $J$ is compatible with $\omega$ or is $\omega$-compatible if $J$ tames $\omega$, and if, in addition, $\omega(J \cdot, J \cdot)=\omega(\cdot, \cdot)$.

Definition 1.14. Let $(M, \omega)$ be a symplectic manifold, let $L \subset M$ be a Lagrangian submanifold, and let $p$ be a point in $L \subset M$. We say that $J$ is relatively integrable at $p$ if there is a holomorphic chart $U \subset M, \alpha: U \rightarrow \mathbb{C}^{n}$ centered at $p$ such that $\alpha^{-1}\left(\mathbb{R}^{n}\right)=U \cap L$.

Definition 1.15. Let $(M, \omega, \phi)$ be a real symplectic manifold with real structure $\phi$. Let $L$ denote $\operatorname{Fix}(\phi)$, and let $p$ be a point in $L$. We say that $J$ is symmetrically integrable at $p$ if there is a holomorphic chart $U \subset M$, $\alpha: U \rightarrow \mathbb{C}^{n}$ centered at $p$ such that $\alpha \circ \phi=c \circ \alpha$.

We first prove the existence of almost complex structures $J$ on a real symplectic manifold $(M, \omega, \phi)$ which tame $\omega$ and satisfy $J \phi_{*}=-\phi_{*} J$. Our discussion follows the methods in Cannas da Silva [8] and McDuff and Salamon $[21]$.

Definition 1.16. Given a symplectic form $\omega$ and an $\omega$-compatible almost complex structure $J$, we denote by $g_{J}: V \times V \rightarrow \mathbb{R}$ the bilinear form defined by

$$
g_{J}(v, w)=\omega(v, J w) .
$$

Lemma 1.17. Let $(V, \omega, \Phi)$ be a real symplectic vector space, i.e., a vector space $V$ with a closed, non-degenerate, skew-symmetric bilinear form $\omega$ and linear map $\Phi$ such that $\Phi^{2}=I$ and $\Phi^{*} \omega=-\omega$. Let $\mathcal{J}_{\Phi}(V, \omega)$ be the space of $\omega$-compatible almost complex structures on $V$ with $\Phi J=-J \Phi$, and let $\mathcal{M e t}_{\Phi}(V)$ denote the space of positive-definite bilinear forms $g$ such that $\Phi^{*} g=g$. Then $\mathcal{J}_{\Phi}(V, \omega)$ is non-empty, and there exists a continuous map $r: \mathcal{M e t}_{\Phi}(V) \rightarrow \mathcal{J}_{\Phi}(V, \omega)$ such that $r\left(g_{J}\right)=J$.

The proof follows [8].

Proof. Let $g \in \operatorname{Met}_{\Phi}(V)$ and define the automorphism $A: V \rightarrow V$ by $\omega(v, w)=g(A v, w)$. Then $\omega(v, w)=-\omega(w, v)$ implies that $g(A v, w)=$ $-g(v, A w)$, and therefore that $A^{*}=-A$. Let $A=Q J$ be the polar decomposition of $A$. Then $Q$ is the unique square root of $A^{*} A$ which is $g$-self-adjoint and $g$-positive-definite. We claim that $J_{g}:=Q^{-1} A$ is a complex structure compatible with $\omega$. First, note that $A$ commutes with $Q^{-1}$, and therefore 
$J_{g}^{2}=Q^{-1} A Q^{-1} A=-\mathrm{Id}$, so $J_{g}$ is an almost complex structure. To see that it is orthogonal, we have

$$
\begin{aligned}
\omega\left(J_{g} v, J_{g} w\right) & =g\left(A Q^{-1} A v, Q^{-1} A w\right) \\
& =g\left(A Q^{-1} A v, Q^{-1} A Q^{-1} A^{*} Q^{-1} A w\right) \\
& =g\left(Q^{-1} A Q^{-1} A v, A Q^{-1} A^{*} Q^{-1} A w\right) \\
& =g\left(-A Q^{-1} A Q^{-1} A v, Q^{-1} A^{*} Q^{-1} A w\right) \\
& =g\left(-A Q^{-1} A Q^{-1} A v,-Q^{-1} A Q^{-1} A w\right) \\
& =g(I A v, I w) \\
& =g(A v, w)=\omega(v, w) .
\end{aligned}
$$

Here, $I$ denotes the identity, and the second and second to last equalities follow because $A^{*}=-A$ and $Q^{-1} A Q^{-1} A=-I$. Also, $\omega\left(v, J_{g} v\right)=g(A v$, $\left.Q^{-1} A v\right)=g\left(v, A^{*} Q^{-1} A v\right)=g\left(v, Q^{-1} A^{*} A v\right)>0$, since both $Q$ and $A^{*} A$ are positive definite. Therefore $J_{g}$ is compatible with $\omega$.

Define $J_{g}:=r_{\Phi}(g)=Q^{-1} A$. For an $\omega$-compatible almost complex structure $J$, we define $g_{J}=\omega(\cdot, J \cdot)$, and we note that

$$
r_{\Phi}\left(g_{J}\right)=r_{\Phi}(\omega(\cdot, J \cdot))=J
$$

since, in this case, $J=A$ and $Q=\mathrm{Id}$.

To see that $\Phi J_{g}=-J_{g} \Phi$, we recall that $\Phi^{*} g=g$ by hypothesis, and we note that $-g(A v, w)=\Phi^{*} \omega(v, w)$, and therefore

$$
-g(A v, w)=\omega(\Phi v, \Phi w)=g(A \Phi v, \Phi w)=g(\Phi A \Phi v, w)
$$

and therefore $\Phi A \Phi=-A$. Now note that $\Phi A^{*} A \Phi=-\Phi A^{2} \Phi=\Phi A \Phi A=$ $-A^{2}=A^{*} A$. Therefore $\Phi Q \Phi=Q$ as well, and $J_{g} \Phi=Q^{-1} A \Phi=-Q^{-1} \Phi A=$ $-\Phi Q^{-1} A=-\Phi J_{g}$, as desired.

To see that the map is continuous, first note that the map $r_{\Phi}$ defined above is the restriction to the set $\operatorname{Met}_{\Phi}(V)$ of the map $r: \operatorname{Met}(V) \rightarrow$ $\mathcal{J}(V, \omega)$ defined in McDuff and Salamon [21, Proposition 2.50(ii)]. Since the restriction of a continuous map is continuous (see, for instance, Munkres [23, Theorem 18.2(d)]), the result follows.

Corollary 1.18. Let $(M, \omega, \phi)$ be a real symplectic manifold. Let $\mathcal{J}_{\phi}(M, \omega)$ denote the space of $\omega$-compatible almost complex structures on $M$ with $\phi_{*} J=$ $-J \phi_{*}$, and let $\mathcal{M e t}_{\phi}(M)$ denote the space of positive-definite bilinear forms $g$ such that $\phi^{*} g=g$. Then $\mathcal{J}_{\Phi}(M, \omega)$ is non-empty and there exists a continuous map $r: \mathcal{M e t}_{\phi}(M) \rightarrow \mathcal{J}_{\phi}(V, \omega)$ such that $r\left(g_{J}\right)=J$. 
Proof. Let $g$ be a $\phi$-invariant Riemannian metric on $M$. Since the polar decomposition is canonical, we may construct an almost complex structure $J$ by constructing $J_{x}$ as in Lemma 1.17 for each $x \in M$. By Lemma 1.17, $J$ is $\omega$-compatible, and $J_{x} \phi_{*}=-\phi_{*} J_{x}$ for each $x \in \operatorname{Fix}(\phi)$.

Now let $x \in M \backslash \operatorname{Fix}(\phi)$. The proof that $\phi_{*} J_{x}=-J_{\phi(x)} \phi_{*}$ also follows the proof of Lemma 1.17. In particular, we first have that $-\omega_{x}(v, w)=$ $\phi^{*} \omega_{x}(v, w)$, and therefore

$$
\begin{aligned}
-g_{x}\left(A_{x} v, w\right) & =-\omega_{x}(v, w)=\omega_{\phi(x)}\left(\phi_{*} v, \phi_{*} w\right) \\
& =g_{\phi(x)}\left(A_{\phi(x)} \phi_{*} v, \phi_{*} w\right) \\
& =g_{x}\left(\phi_{*} A_{\phi(x)} \phi_{*} v, w\right)
\end{aligned}
$$

for all $v, w \in T_{x} M$, and therefore $\phi_{*} A_{\phi(x)} \phi_{*}=-A_{x}$. Now note that

$$
\phi_{*} A_{\phi(x)}^{*} A_{\phi(x)} \phi_{*}=-\phi_{*} A_{\phi(x)}^{2} \phi_{*}=\phi_{*} A_{\phi(x)} \phi_{*} A_{x}=-A_{x}^{2}=A_{x}^{*} A_{x} .
$$

Therefore $\phi_{*} Q_{\phi(x)} \phi_{*}=Q_{x}$ as well, and

$$
J_{\phi(x)} \phi_{*}=Q_{\phi(x)}^{-1} A_{\phi(x)} \phi_{*}=-Q_{\phi(x)}^{-1} \phi_{*} A_{x}=-\phi_{*} Q_{x}^{-1} A_{x}=-\Phi J_{x},
$$

as desired.

Remark 1.19. Indeed, this corollary shows that, for a real symplectic manifold $(M, \omega, \phi)$, there exists a $\omega$-compatible (and therefore tame) almost complex structure $J$ with $\phi_{*} J=-J \phi_{*}$.

Remark 1.20. Note that if $\psi:\left(B(1+2 \epsilon), \lambda^{2} \omega_{0}, B_{\mathbb{R}}(1+2 \epsilon)\right) \rightarrow(M, \omega, L)$ is a relative or real symplectic embedding, then the above constructions imply that there exists an $\omega$-tame (compatible) almost complex structure $J$ which equals $\psi_{*} i \psi_{*}^{-1}$ on a neighborhood of $\psi(0)$, and therefore $J$ is symmetrically or relatively integrable at $\psi(0)$ if $\psi$ is a real or relative embedding, respectively. If, in addition, $M$ has a real structure $\phi$ and $\psi$ is a real symplectic embedding, then $J$ also may be taken to satisfy $\phi_{*} J \phi_{*}=-J$. Similarly, if $\tilde{\psi}:(\mathcal{L}(1+2 \epsilon), \rho(1, \delta), \mathcal{R}(1+2 \epsilon)) \rightarrow(\tilde{M}, \tilde{\omega}, \tilde{L})$ is a real or relative embedding, then there exists an $\tilde{\omega}$-tame almost complex structure $\tilde{J}$ such that $\tilde{J}=\tilde{\psi}_{*} \tilde{i} \tilde{\psi}_{*}^{-1}$ in a neighborhood of $\mathcal{L}(0)$.

\subsection{Main results}

We now state our main theorems, using the notation in Section 1.1. Theorem 1.21 is proved in Section 2, the proof of 1.22 is completed in Section 3. 
Theorem 1.21 (Blow-up). (1) Let $(M, \omega)$ be a symplectic manifold and let $L \subset M$ be a Lagrangian submanifold. Suppose that for some small $\epsilon>0$ there is a $(p, q)$-mixed symplectic embedding

$$
\psi: \coprod_{j=1}^{k}\left(B_{j}(1+2 \epsilon), \lambda_{j}^{2} \omega_{0}, B_{\mathbb{R}, j}(1+2 \epsilon)\right) \hookrightarrow(M, \omega, L),
$$

let $P \subset M$ be the set $P:=\left\{\psi_{j}(0)\right\}_{j=1}^{k}$, and let $J$ be an $\omega$-tame (compatible) almost complex structure which is locally symmetrically integrable in a neighborhood of $P$.

Then there exists a manifold $\tilde{M}$, a family of symplectic forms $\tilde{\omega}_{t}, t \in$ $[0,1]$ on $\tilde{M}$, a submanifold $\tilde{L} \subset \tilde{M}$ which is Lagrangian for each $\tilde{\omega}_{t}$, and a smooth onto map $\Pi: \tilde{M} \rightarrow M$ such that the following is satisfied:

(a) $\Pi$ is a diffeomorphism on $\Pi^{-1}(M \backslash P)$,

(b) $\Pi^{-1}\left(\psi_{j}(0)\right) \cong \mathbb{C} P^{n-1}$,

(c) $\Pi(\tilde{L})=L$,

(d) $\tilde{\omega}_{0}$ tames (is compatible with) an almost complex structure $\tilde{J}$ for which each $\Pi^{-1}\left(\psi_{j}(0)\right)$ is an almost complex manifold, and

(e) $\tilde{\omega}_{1}$ is in the cohomology class

$$
\left[\tilde{\omega}_{1}\right]=\left[\Pi^{*} \omega\right]-\sum_{j=1}^{k} \pi \lambda_{j}^{2} e_{j},
$$

where the $e_{j}$ are the Poincare duals of the exceptional classes $E_{j}=$ $\left[\Pi^{-1}\left(\psi_{j}(0)\right)\right]$.

(2) If, in addition, $M$ admits an anti-symplectic involution $\phi$ which satisfies

(a) $\operatorname{Fix}(\phi)=L$,

(b) $\operatorname{Im}(\phi \circ \psi)=\operatorname{Im}(\psi)$,

(c) $\operatorname{Im}\left(\phi \circ \psi_{j}\right) \cap \operatorname{Im}\left(\psi_{j}\right)=\emptyset$ if $\operatorname{Im}\left(\psi_{j}\right) \cap L=\emptyset$, and

(d) $\psi_{j} \circ c=\phi \circ \psi_{j}$ if $\operatorname{Im}\left(\psi_{j}\right) \cap L \neq \emptyset$,

then $\tilde{M}$ admits an involution $\tilde{\phi}: \tilde{M} \rightarrow \tilde{M}$ such that $\tilde{\phi}^{*} \tilde{\omega}_{t}=-\tilde{\omega}_{t}, \phi \circ$ $\Pi=\Pi \circ \tilde{\phi}$, and $\tilde{\phi}_{*} \tilde{J} \tilde{\phi}_{*}=\tilde{J}$.

Theorem 1.22 (Blow-down). (1) Let $(\tilde{M}, \tilde{\omega})$ be a symplectic manifold with Lagrangian $\tilde{L}$. Suppose there is a $(p, q)$-mixed symplectic embedding

$$
\tilde{\psi}: \coprod_{j=1}^{k}\left(\mathcal{L}_{j}\left(r_{j}\right), \rho_{j}\left(\delta_{j}, \lambda_{j}\right), \mathcal{R}_{j}\left(r_{j}\right)\right) \hookrightarrow(\tilde{M}, \tilde{\omega}, \tilde{L})
$$


such that $\psi^{-1}(\tilde{L})=\coprod_{j=1}^{p} \mathcal{R}_{j}\left(r_{j}\right)$. Let $C_{j} \subset \tilde{M}$ denote $\tilde{\psi}_{j}(\mathcal{L}(0))$, and let $C=\cup_{j} C_{j}$.

Then there exists a symplectic manifold $(M, \omega)$, a $(p, q)$-mixed symplectic embedding

$$
\psi: \coprod_{j=1}^{k}\left(B_{j}(1+2 \epsilon), \lambda_{j} \omega_{0}, B_{j, \mathbb{R}}(1+2 \epsilon)\right) \rightarrow(M, \omega, L),
$$

a Lagrangian submanifold $L \subset M$, and a smooth onto map $\Pi: \tilde{M} \rightarrow$ $M$ such that the following is satisfied:

(a) $\Pi$ is a diffeomorphism on $\tilde{M} \backslash C$,

(b) $\Pi\left(C_{j}\right)=p_{j} \in M$, where $p_{j}$ is a point,

(c) $\Pi(\tilde{L})=L$, and

(d) $\omega$ satisfies

$$
[\tilde{\omega}]-\left[\Pi^{*} \omega\right] \in \mathcal{E}
$$

where $\mathcal{E}$ is the linear vector space generated by $e_{1}, \ldots, e_{k}$, the Poincaré duals of the exceptional classes $E_{j}=\left[\tilde{\psi}_{j}(0)\right]$.

(2) Suppose, in addition, $\tilde{M}$ admits an anti-symplectic involution $\tilde{\phi}$ which satisfies

(a) $\operatorname{Fix}(\tilde{\phi})=\tilde{L}$,

(b) $\operatorname{Im}(\tilde{\psi})=\operatorname{Im}(\tilde{\phi} \circ \tilde{\psi})$,

(c) $\operatorname{Im}\left(\tilde{\phi} \circ \tilde{\psi}_{j}\right) \cap \operatorname{Im}\left(\tilde{\psi}_{j}\right)=\emptyset$ if $\operatorname{Im}\left(\psi_{j}\right) \cap L=\emptyset$, and

(d) $\tilde{\psi}_{j} \circ \tilde{c}=\tilde{\phi} \circ \tilde{\psi}_{j}$ if $\operatorname{Im}\left(\tilde{\psi}_{j}\right) \cap \tilde{L} \neq \emptyset$.

Then $(M, \omega)$ admits an anti-symplectic involution $\phi$ such that $\phi \circ$ $\Pi=\Pi \circ \tilde{\phi}$.

The idea of the relative blow-up construction is the same as blowing up in the purely symplectic case: we remove the interior of a ball from both $M$ and $\overline{\mathbb{C P}}^{n}$ (the bar indicating that the orientation is reversed), and we glue them along their boundaries, ensuring that the symplectic form $\tilde{\omega}$ of the blow-up $\tilde{M}$ acts appropriately. The difference in the relative case is that the real parts of the balls removed from $M$ and $\overline{\mathbb{C P}}^{n}$ are constrained to intersect the Lagrangians $L$ and $\mathbb{R} P^{n}$, respectively, and the gluing proceeds so that the boundary of the ( $n$-dimensional) ball removed from $L$ is then glued to the boundary of the corresponding hole in $\mathbb{R} P^{n}$, resulting in the new Lagrangian $L \# \mathbb{R} P^{n} \cong \tilde{L} \subset \tilde{M}$ in the blow-up. The blow-down is the reverse process. We make these operations precise in Section 2.

In four-dimensional complex geometry and symplectic topology, it is extremely useful to know that one can blow-down a symplectic manifold $M$ 
along an embedded $J$-holomorphic sphere $C$ when $[C] \cdot[C]=-1$. In complex geometry this is the so-called Castelnuovo-Enriques criterion (see, for example, [10, p. 476]). Unfortunately, it is a difficult problem in general to derive a condition to detect when blowing-down $C$ can be arranged to change the topology of a (non-orientable) Lagrangian $\tilde{L}$. However, for Lagrangian submanifolds which are the fixed point set of an anti-symplectic involution $\phi$ on a symplectic 4-manifold $M$, we have the following result, which we prove in Section 3.

We first give the following definition.

Definition 1.23. We call $E \in H_{2}\left(M^{4} ; \mathbb{Z}\right)$ an exceptional class if $E \cdot E=$ -1 . If $u: \Sigma \hookrightarrow M^{4}$ is an embedding of the surface $\Sigma$, and $u_{*}[\Sigma]=E$, then we say that $u(\Sigma)$ is an exceptional curve.

Theorem 1.24. Let $\left(M^{4}, \omega, \phi\right)$ be a real symplectic manifold with $L:=$ $\operatorname{Fix}(\phi)$, and let $J$ be an almost complex structure on $M$ which tames $\omega$ and which satisfies $\phi_{*} J \phi_{*}=-J$. Suppose $E \in H_{2}(M ; \mathbb{Z})$ satisfies $E \cdot E=$ -1 and $\phi_{*} E=-E$, and that there exists an embedded J-holomorphic curve $C$ which represents $E$. Then there exists a real symplectic manifold $(\check{M}, \check{\omega}, \check{\phi})$ and a smooth onto map $\Pi: M \rightarrow \check{M}$ that satisfies

(1) $\Pi$ is a diffeomorphism on $M \backslash C$,

(2) $\Pi(C)=p \in \check{M}$, where $p$ is a point,

(3) $\Pi \circ \phi=\check{\phi} \circ \Pi$, and

(4) $\check{\omega}$ satisfies

$$
[\omega]-\left[\Pi^{*} \check{\omega}\right] \in \mathcal{E}
$$

where $\mathcal{E}$ is the linear vector space generated by e, the Poincaré dual of the exceptional class $E=\left[\Pi^{-1}(p)\right]$.

As an application of the above theorems, we have the following theorem on the real packing numbers for $\left(\mathbb{C} P^{2}, \mathbb{R} P^{2}\right)$, defined below.

Definition 1.25. Let $(M, \omega)$ be a symplectic manifold with Lagrangian submanifold $L \subset M$. We call the number

$$
p_{L, k}:=\sup _{\psi} \frac{\operatorname{Vol}\left(\coprod_{i=1}^{k}\left(B(\lambda), \omega_{0}, B_{\mathbb{R}}(\lambda)\right)\right)}{\operatorname{Vol}(M)},
$$


the $k$ th relative packing number for $(M, L)$, where the sup is taken over all relative symplectic embeddings

$$
\psi: \coprod_{i=1}^{k}\left(B(\lambda), \omega_{0}, B_{\mathbb{R}}(\lambda)\right) \rightarrow(M, \omega, L) .
$$

If $M$ is a real manifold with real structure $\phi, \operatorname{Fix}(\phi)=L$, and the sup is taken over all real embeddings of $k$ balls, then $p_{L, k}$ is called the $k$ th real packing number. We will also denote the $k$ th real packing number by $p_{\mathbb{R}, k}$. If the supremum is taken over all symplectic embeddings of $k$ balls into $M$, then we denote the number $p_{k}$ and we call it the $k$ th packing number of $M$.

Theorem 1.26. For the pair $\left(\mathbb{C} P^{2}, \mathbb{R} P^{2}\right)$ with the standard symplectic form and real structure, the real packing numbers $p_{\mathbb{R} P^{2}, k}$ are equal to the absolute packing numbers for $\mathbb{C} P^{2}$.

\section{Constructing the relative and real blow-up and blow-down}

We now construct the blow-up and blow-down of a symplectic manifold $(M, \omega)$ relative to a Lagrangian submanifold $L$ or a real structure $\phi$. The general strategy is to perform a complex blow-up or blow-down locally and then define a symplectic form for the resulting manifold. In each case, we first discuss the local models for the symplectic forms in these constructions, and we then construct the global blow-up and blow-down given a mixed, relative or real symplectic embedding

$$
\begin{aligned}
& \psi: \coprod_{j=1}^{k}\left(B_{j}(1+2 \epsilon), \lambda_{j}^{2} \omega_{0}, B_{\mathbb{R}, j}(1+2 \epsilon)\right) \hookrightarrow(M, \omega, L), \text { or } \\
& \tilde{\psi}: \coprod_{j=1}^{k}\left(\mathcal{L}_{j}(1+2 \epsilon), \rho\left(\delta, \lambda_{j}\right), \mathcal{R}_{j}\right) \hookrightarrow(\tilde{M}, \tilde{\omega}, \tilde{L})
\end{aligned}
$$

and the local models.

\subsection{Blow-up}

In this section, we prove Theorem 1.21, which we restate here for the convenience of the reader.

Theorem (Theorem 1.21). (1) Let $(M, \omega)$ be a symplectic manifold and let $L \subset M$ be a Lagrangian submanfiold. Suppose that for some 
small $\epsilon>0$ there is a $(p, q)$-mixed symplectic embedding

$$
\psi: \coprod_{j=1}^{k}\left(B_{j}(1+2 \epsilon), \lambda_{j}^{2} \omega_{0}, B_{\mathbb{R}, j}(1+2 \epsilon)\right) \hookrightarrow(M, \omega, L)
$$

let $P \subset M$ be the set $P:=\left\{\psi_{j}(0)\right\}_{j=1}^{k}$, and let $J$ be an $\omega$-tame (compatible) almost complex structure which is locally symmetrically integrable in a neighborhood of $P$.

Then there exists a manifold $\tilde{M}$, a family of symplectic forms $\tilde{\omega}_{t}, t \in$ $[0,1]$ on $\tilde{M}$, a submanifold $\tilde{L} \subset \tilde{M}$ which is Lagrangian for each $\tilde{\omega}_{t}$, and a smooth onto map $\Pi: \tilde{M} \rightarrow M$ such that the following is satisfied:

(a) $\Pi$ is a diffeomorphism on $\Pi^{-1}(M \backslash P)$,

(b) $\Pi^{-1}\left(\psi_{j}(0)\right) \cong \mathbb{C} P^{n-1}$,

(c) $\Pi(\tilde{L})=L$,

(d) $\tilde{\omega}_{0}$ tames (is compatible with) an almost complex structure $\tilde{J}$ for which each $\Pi^{-1}\left(\psi_{j}(0)\right)$ is an almost complex manifold, and

(e) $\tilde{\omega}_{1}$ is in the cohomology class

$$
\left[\tilde{\omega}_{1}\right]=\left[\Pi^{*} \omega\right]-\sum_{j=1}^{k} \pi \lambda_{j}^{2} e_{j}
$$

where the $e_{j}$ are the Poincaré duals of the exceptional classes $E_{j}=$ $\left[\Pi^{-1}\left(\psi_{j}(0)\right)\right]$.

(2) If, in addition, $M$ admits an anti-symplectic involution $\phi$ which satisfies

(a) $\operatorname{Fix}(\phi)=L$,

(b) $\operatorname{Im}(\phi \circ \psi)=\operatorname{Im}(\psi)$,

(c) $\operatorname{Im}\left(\phi \circ \psi_{j}\right) \cap \operatorname{Im}\left(\psi_{j}\right)=\emptyset$ if $\operatorname{Im}\left(\psi_{j}\right) \cap L=\emptyset$, and

(d) $\psi_{j} \circ c=\phi \circ \psi_{j}$ if $\operatorname{Im}\left(\psi_{j}\right) \cap L \neq \emptyset$,

then $\tilde{M}$ admits an involution $\tilde{\phi}: \tilde{M} \rightarrow \tilde{M}$ such that $\tilde{\phi}^{*} \tilde{\omega}_{t}=-\tilde{\omega}_{t}, \phi \circ$ $\Pi=\Pi \circ \tilde{\phi}$, and $\tilde{\phi}_{*} \tilde{J} \tilde{\phi}_{*}=\tilde{J}$.

The construction proceeds as follows. We first construct a family of symplectic forms $\tilde{\tau}(\epsilon, \lambda)$ on $\mathcal{L}$ by pulling back the standard form $\omega_{0}$ on $\mathbb{R}^{2 n}$ by a family of specially constructed maps from $\mathcal{L} \rightarrow \mathbb{R}^{2 n}$. We arrange, in particular, that the submanifold $\mathcal{R} \subset \mathcal{L}$ is a Lagrangian for the forms $\tilde{\tau}(\epsilon, \lambda)$. We then consider a relative symplectic and holomorphic embedding $\psi$ : $\left(B(1+2 \epsilon), \lambda^{2} \omega_{0}, B_{\mathbb{R}}(1+2 \epsilon), i\right) \rightarrow(M, \omega, L, J)$, and we construct the blowup manifold $(\tilde{M}, \tilde{L})$ by removing the ball and gluing in $(\mathcal{L}(1+2 \epsilon), \mathcal{R}(1+2 \epsilon))$ 
along the boundary. Finally, we use the local forms $\tilde{\tau}(\epsilon, \lambda)$ created on $\mathcal{L}$ in the first step to construct the global symplectic form $\tilde{\omega}$ on the blow-up $\tilde{M}$. For a real manifold $M$, we also construct a real structure on the blow-up $\tilde{M}$. We then show that, given a relative symplectic embedding, and in view of some appropriate (and non-restrictive) assumptions on the almost complex structures, we may find a holomorphic embedding of a smaller ball which is compatible with $L$ (or a real structure $\phi$ ), and we use this to remove the assumption of holomorphicity on the embeddings.

In the following proposition, we construct the forms $\tilde{\tau}(\epsilon, \lambda)$. Note that points (1), (2), and (3) of the proposition were proved in Proposition 5.1.A of McDuff and Polterovich [20].

Proposition 2.1. Using the notation in Section 1.1, for every $\epsilon, \lambda>0$ there exists a symplectic form $\tilde{\tau}(\epsilon, \lambda)$ on $\mathcal{L}$ such that the following holds:

(1) $\tilde{\tau}(\epsilon, \lambda)=\pi^{*}\left(\lambda^{2} \omega_{0}\right)$ on $\mathcal{L}-\mathcal{L}(1+\epsilon)$,

(2) $\tilde{\tau}(\epsilon, \lambda)=\rho(1, \lambda)$ on $\mathcal{L}(\delta)$ for some $\delta>0$,

(3) $\tilde{\tau}(\epsilon, \lambda)$ is compatible with $\tilde{i}$, the canonical integrable complex structure on $\mathcal{L}$,

(4) $\tilde{c}^{*} \tilde{\tau}(\epsilon, \lambda)=-\tilde{\tau}(\epsilon, \lambda)$, where $\tilde{c}$ denotes complex conjugation on $\mathcal{L}$,

(5) $\left.\tilde{\tau}(\epsilon, \lambda)\right|_{\mathcal{R}}=0$.

The proof of this proposition will be based on the following three lemmas. Lemma 2.3 was proved in [11], although it may also be proved by a direct, if long, calculation. Lemma 2.4 is a well-known result which we state so we may refer to it later, and Lemma 2.5 was stated and the proof sketched in [20]. The details of the proof are a routine calculation. We begin with a definition.

Definition 2.2. We say that $f: \mathbb{C}^{n} \rightarrow \mathbb{C}^{n}$ is a radial function if $f(z)=$ $\alpha(|z|) z$ for some real-valued function $\alpha: \mathbb{R} \rightarrow[0, \infty)$. We say that a radial function $f$ is monotone if $\left|z_{0}\right| \leq\left|z_{1}\right| \Longrightarrow\left|f\left(z_{0}\right)\right| \leq\left|f\left(z_{1}\right)\right|$.

Lemma 2.3. Let $h: \mathbb{R}^{2 n} \rightarrow \mathbb{R}$ be the function $h(x)=\left(1+\frac{\lambda^{2}}{|x|^{2}}\right)^{1 / 2}$ and $\omega_{0}$ be the standard symplectic form on $\mathbb{R}^{2 n}$. Let $H: \mathbb{R}^{2 n} \backslash\{0\} \rightarrow \mathbb{R}^{2 n} \backslash B(\lambda)$ be the mapping given by $H(x)=h(x) x$. Then $\pi^{*} H^{*} \omega=\rho(1, \lambda)$ on $\mathcal{L} \backslash\{(0, l) \mid l \in$ $\left.\mathbb{C} P^{n-1}\right\}$. 
Lemma 2.4. Let $(M, \omega)$ be a symplectic manifold. Then $\omega$ is a Kähler form iff $\omega$ is compatible with an integrable almost complex structure $J$.

Lemma 2.5. Let $\omega$ be a Kähler form on $\mathbb{C}^{n}$, and suppose $f: \mathbb{C}^{n} \backslash\{0\} \rightarrow$ $\mathbb{C}^{n} \backslash\{0\}$ is a monotone radial diffeomorphism. Then $f^{*} \omega$ is a Kähler form.

We now prove the following proposition, which we follow with the proof of Proposition 2.1.

Proposition 2.6. For each $\kappa, \lambda>0, \rho(\kappa, \lambda)$ is a symplectic form on $\mathcal{L}$.

Proof. Let $\Omega=\omega_{0}^{n}$ denote the volume form on $\mathbb{R}^{2 n}$, and let $H$ be defined as in the proof of Lemma 2.3. Since $H \circ \pi$ is a diffeomorphism on $\mathcal{L}^{*}:=$ $\mathcal{L} \backslash\left\{(0, z) \mid z \in \mathbb{C} P^{n-1}\right\}, \pi^{*} H^{*} \Omega$ is a volume form on $\mathcal{L}$, and therefore $\rho(1, \lambda)$ is non-degenerate for any $\lambda>0$. Since $\rho(\kappa, \lambda)=\kappa^{2} \rho(1, \lambda / \kappa)$, this implies that $\rho(\kappa, \lambda)$ is non-degenerate for $\kappa, \lambda>0$ as well. Since both $\omega_{0}$ and $\sigma$ are closed, $\rho(\kappa, \lambda)$ is closed as well on $\mathcal{L}^{*}$.

Now let $(0, l) \in \mathcal{L}(0)$. Then $T_{(0, l)} \mathcal{L} \equiv T_{l} \mathbb{C} P^{1} \oplus T_{0} \mathbb{C}$. Taking $v \in T_{l} \mathbb{C} P^{1}$, we have $\rho(\kappa, \lambda)(v, i v)=\lambda^{2} \theta^{*} \sigma(v, i v)=\sigma(v, i v)>0$. Similarly, for $v \in T_{0} \mathbb{C}$, $\rho(\kappa, \lambda)(v, i v)=\pi^{*} \omega_{0}(v, i v)>0$, and therefore $\rho(\kappa, \lambda)$ is non-degenerate on $\mathcal{L}(0)$. Since $\rho(\kappa, \lambda)$ is closed as well, the form is symplectic as desired.

Proof of Proposition 2.1. For each $\lambda>0$, let $h_{\lambda}: \mathbb{R}^{2 n} \backslash\{0\} \rightarrow \mathbb{R}$ be given by $h_{\lambda}(x)=\left(1+\frac{\lambda^{2}}{|x|^{2}}\right)^{1 / 2}$, and let $\delta, \epsilon, \epsilon_{0}>0$ satisfy $\left(\delta+\epsilon_{0}\right)^{2}<\lambda^{2} \epsilon / 2$. For $x \in$ $B\left(\delta+\epsilon_{0}\right)$, we therefore have $\left|h_{\lambda}(x) x\right|^{2}=|x|^{2}+\lambda^{2} \leq\left(\delta+\epsilon_{0}\right)^{2}+\lambda^{2}<\lambda^{2}$ $(\epsilon / 2+1)$. Let $\beta(t): \mathbb{R} \rightarrow \mathbb{R}$ be a smooth non-increasing function which is 1 for $t \leq \delta$ and 0 for $t \geq \delta+\epsilon_{0}$, and let $\gamma(t): \mathbb{R} \rightarrow \mathbb{R}$ be a smooth nonincreasing function which is 1 for $t \leq(1+\epsilon)^{1 / 2}$ and 0 for $t \geq 1+\epsilon$. Now define $F: \mathbb{R}^{2 n} \backslash\{0\} \rightarrow \mathbb{R}^{2 n}$ by

$$
F(x)= \begin{cases}h_{\lambda}(x) x, & |x|<\delta, \\ \beta(|x|) h_{\lambda}(x) x+\left(1-\beta(|x|) \lambda\left(1+\frac{\epsilon}{2}\right)^{\frac{1}{2}} \frac{x}{|x|},\right. & \delta \leq|x| \leq(1+\epsilon)^{\frac{1}{2}}, \\ \gamma(|x|) \lambda\left(1+\frac{\epsilon}{2}\right)^{\frac{1}{2}} \frac{x}{|x|}+(1-\gamma(|x|)) \lambda x, & (1+\epsilon)^{\frac{1}{2}}<|x|<1+\epsilon, \\ \lambda x, & 1+\epsilon \leq|x| .\end{cases}
$$

Lemma 2.7. The function $F$ defined above is a monotone radial diffeomorphism.

Proof. We first note that $F$ is radial by definition. Furthermore, since the function is a diffeomorphism on each region, and since $\beta$ and $\gamma$ are smooth 
and all of their derivatives vanish on the boundary of each region, it follows that $F$ is continuous and all of the derivatives of $F$ are well defined for all $t$, and therefore $F$ is a diffeomorphism. We now show that $F$ is monotone. For $|x|<\delta$, we have that $|F(x)|^{2}=|x|^{2}+\lambda^{2}$, so $F$ is monotone on this region. For $\delta \leq|x| \leq(1+\epsilon)^{1 / 2}$, we have

$$
\begin{aligned}
|F(x)| & =\mid \beta(|x|) h_{\lambda}(x) x+\left(1-\beta(|x|) \lambda\left(1+\frac{\epsilon}{2}\right)^{1 / 2} \frac{x}{|x|} \mid\right. \\
& =\mid \beta(|x|) h_{\lambda}(x)+\left(1-\beta(|x|) \lambda\left(1+\frac{\epsilon}{2}\right)^{1 / 2} \frac{1}{|x|}|| x \mid\right. \\
& =\beta(|x|)\left(|x|^{2}+\lambda^{2}\right)^{1 / 2}+\left(1-\beta(|x|) \lambda\left(1+\frac{\epsilon}{2}\right)^{1 / 2} .\right.
\end{aligned}
$$

Here, the last equality follows since each term in the coefficient of $|x|$ is always a positive real number. Setting $t:=|x|$ and $G(t):=|F(x)|$, we now compute

$$
\begin{aligned}
\frac{d G}{d t} & =\beta(t) \frac{t}{\left(t^{2}+\lambda^{2}\right)^{1 / 2}}+\left(t^{2}+\lambda^{2}\right)^{1 / 2} \beta^{\prime}(t)-\lambda\left(1+\frac{\epsilon}{2}\right)^{1 / 2} \beta^{\prime}(t) \\
& =\beta(t) \frac{t}{\left(t^{2}+\lambda^{2}\right)^{1 / 2}}+\left(\left(t^{2}+\lambda^{2}\right)^{1 / 2}-\lambda\left(1+\frac{\epsilon}{2}\right)^{1 / 2}\right) \beta^{\prime}(t) .
\end{aligned}
$$

The first term in the last equality is positive for all $t>0$. For $t \geq \delta+\epsilon_{0}$, $\beta^{\prime}(t)=0$, and for $\delta<t<\delta+\epsilon_{0}, \beta^{\prime}(t) \leq 0$. On this region, we also have that

$$
\begin{aligned}
t^{2}+\lambda^{2} & <\left(\delta+\epsilon_{0}\right)^{2}+\lambda^{2} \\
& <\lambda^{2} \epsilon / 2+\lambda^{2} \\
& =\lambda^{2}\left(1+\frac{\epsilon}{2}\right) .
\end{aligned}
$$

Taking square roots of both sides of the inequality, we see that the coefficient to $\beta^{\prime}(t)$ in equation $(2.2)$ is negative. Therefore, $\frac{d G}{d t}$ is non-negative for $t \in$ $\left(\delta,(1+\epsilon)^{1 / 2}\right)$, and it follows that $|F(x)|$ is non-decreasing there.

Similarly, for $|x| \in\left((1+\epsilon)^{1 / 2}, 1+\epsilon\right)$, we have

$$
|F(x)|=\gamma(|x|) \lambda\left(1+\frac{\epsilon}{2}\right)^{1 / 2}+(1-\gamma(|x|)) \lambda|x| .
$$

Setting $t:=|x|$, and $G(t):=|F(x)|$, we compute

$$
\frac{d G}{d t}=\left(\lambda\left(1+\frac{\epsilon}{2}\right)^{1 / 2}-\lambda t\right) \gamma^{\prime}(t)+(1-\gamma(|x|)) \lambda .
$$


The last term is positive, and, since $\gamma^{\prime}(t) \leq 0$ and $t>\left(1+\frac{\epsilon}{2}\right)^{1 / 2}$, the first term is positive as well. Therefore $G(t)$ is non-decreasing, and $|F(x)|$ is monotone on this interval. For $|x| \in[1+\epsilon, \infty),|F(x)|$ is clearly monotone. This completes the proof.

We now return to the proof of Proposition 2.1. Define $\tilde{\tau}(\epsilon, \lambda)$ by

$$
\tilde{\tau}(\epsilon, \lambda):=\pi^{*} F^{*} \omega_{0}
$$

on $\mathcal{L} \backslash \mathcal{L}(0)$. By Lemma $2.3, \pi^{*} F^{*} \omega_{0}=\rho(1, \lambda)=\tilde{\tau}(\epsilon, \lambda)$ on $\mathcal{L}(\delta) \backslash \mathcal{L}(0)$. Since $\rho(1, \lambda)$ is a symplectic form on all of $\mathcal{L}(\delta)$, we may extend $\tilde{\tau}(\epsilon, \lambda)$ to all of $\mathcal{L}$ by assigning $\tilde{\tau}(\epsilon, \lambda):=\rho(1, \lambda)$ on $\mathcal{L}(0)=\pi^{-1}(0)$. Now note that this form satisfies conditions 1 and 2 in the proposition by Lemma 2.3 and the definition of $F$.

To see that $\tilde{\tau}(\epsilon, \lambda)$ is symplectic, we note that on $\mathcal{L} \backslash \mathcal{L}(0), \tilde{\tau}(\epsilon, \delta)$ is a pullback of the symplectic form $\omega_{0}$ by the diffeomorphism $F$, and on a neighborhood of $\mathcal{L}(0), \tau(\epsilon, \lambda)$ equals the symplectic form $\rho(1, \lambda)$.

Items 3,4 , and 5 follow from a routine calculation.

In the next proposition, we construct the global relative blow-up of a manifold $M$ using a relative symplectic and holomorphic embedding of the ball $\left(B(1+2 \epsilon), \lambda^{2} \omega_{0}, B_{\mathbb{R}}(1+2 \epsilon)\right)$ with the standard complex structure $i$. The use of holomorphic embeddings here gives us extra control over the complex structure in the blow-up, which we will be useful in our applications.

Proposition 2.8. Let $(M, \omega)$ be a symplectic manifold with Lagrangian $L$, and let $J$ be an $\omega$-tame (compatible) almost complex structure. Suppose that for $\lambda>0$ and some small $\epsilon>0$, there is a relative symplectic and holomorphic embedding

$$
\psi: \coprod_{j=1}^{k}\left(B_{j}(1+2 \epsilon), \lambda_{j}^{2} \omega_{0}, B_{\mathbb{R}, j}(1+2 \epsilon), i\right) \hookrightarrow(M, \omega, L, J) .
$$

Then there exists a symplectic manifold $(\tilde{M}, \tilde{\omega})$ with Lagrangian $\tilde{L} \subset \tilde{M}$, an $\tilde{\omega}$-tame (compatible) almost complex structure $\tilde{J}$, and a smooth onto map $\Pi: \tilde{M} \rightarrow M$ such that

(1) $\Pi$ is a diffeomorphism on $\Pi^{-1}\left(M \backslash \cup_{j=1}^{k} \psi_{j}(0)\right)$,

(2) $\Pi_{*} \tilde{J}=J \Pi_{*}$,

(3) for all $j \in\{1, \ldots, k\}, \Pi^{-1}\left(\psi_{j}(0)\right) \cong \mathbb{C} P^{n-1}$, 
(4) $\Pi(\tilde{L})=L$, and

(5) $\tilde{\omega}$ is in the cohomology class

$$
[\tilde{\omega}]=\left[\Pi^{*} \omega\right]-\sum_{j=1}^{k} \pi \lambda_{j}^{2} e_{j}
$$

where the $e_{j}$ are the Poincaré duals of the exceptional classes

$$
E_{j}=\left[\Pi^{-1}\left(\psi_{j}(0)\right)\right]
$$

Remark 2.9. Note that the $E_{i}$ in the theorem above are the classes represented by the exceptional curves added in the blow-up.

Proof. First, we consider the case when $k=1$. Consider the map $\pi:(\mathcal{L}(1+$ $2 \epsilon), \mathcal{R}(1+2 \epsilon), \tilde{i}) \rightarrow\left(B(1+2 \epsilon), B_{\mathbb{R}}(1+2 \epsilon), i\right)$ from Definition 1.2 , where $\tilde{i}$ and $i$ are the standard complex structures on $\mathcal{L}$ and $\mathbb{C}^{n}$, respectively. Observing that $\pi$ gives a diffeomorphism between the boundaries $(\partial B(1+2 \epsilon)$, $\left.\partial B_{\mathbb{R}}(1+2 \epsilon)\right)$, and $(\partial \mathcal{L}(1+2 \epsilon), \partial \mathcal{R}(1+2 \epsilon))$, we let $\pi_{\partial}$ denote the restriction of $\pi$ to $\partial \mathcal{L}(1+2 \epsilon)$, and we define $\tilde{M}$ to be $\tilde{M}:=M \backslash \psi\left(\left(B(1+2 \epsilon), B_{\mathbb{R}}(1+\right.\right.$ $2 \epsilon)) \cup_{\psi \circ \pi_{\partial}}(\mathcal{L}(1+2 \epsilon), R(1+2 \epsilon))$. This operation is summarized in the diagram below, with $\delta=1+2 \epsilon$.

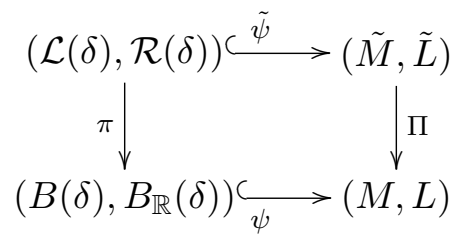

where $\psi$ and $\tilde{\psi}$ are embeddings, and where the map $\Pi:(\tilde{M}, \tilde{L}) \rightarrow(M, L)$ is defined by

$$
\Pi(x)= \begin{cases}x, & x \notin \operatorname{Im} \tilde{\psi}, \\ \psi \circ \pi \circ \tilde{\psi}^{-1}(x), & x \in \operatorname{Im} \tilde{\psi},\end{cases}
$$

making the diagram commutative. Note that only $\psi$ is a symplectomorphism a priori.

We now define a symplectic form on $\tilde{M}$. Recall that $\psi^{*} \omega=\lambda^{2} \omega_{0}$ by hypothesis. We assign a symplectic form to $\tilde{M}$ by

$$
\tilde{\omega}= \begin{cases}\Pi^{*} \omega & \text { on } \tilde{M} \backslash \tilde{\psi}(\mathcal{L}(1+\epsilon)), \\ \left(\tilde{\psi}^{-1}\right)^{*} \tilde{\tau}(\epsilon, \lambda) & \text { on } \tilde{\psi}(\mathcal{L}(1+2 \epsilon)) .\end{cases}
$$


We check that $\tilde{\omega}$ is well defined on $\mathcal{L}(1+2 \epsilon)-\mathcal{L}(1+\epsilon)$. By Proposition 2.1 and the definition of $\tilde{\omega}$ and $\Pi$, on $\mathcal{L}(1+2 \epsilon)-\mathcal{L}(1+\epsilon)$ we have

$$
\begin{aligned}
\Pi^{*} \omega & =\left(\tilde{\psi}^{-1}\right)^{*} \pi^{*} \psi^{*} \omega \\
& =\lambda^{2}\left(\tilde{\psi}^{-1}\right)^{*} \pi^{*} \omega_{0}=\left(\tilde{\psi}^{-1}\right)^{*} \tilde{\tau}(\epsilon, \lambda),
\end{aligned}
$$

so $\tilde{\omega}$ is well defined.

We define the almost complex structure $\tilde{J}$ on $\tilde{M}$ by

$$
\tilde{J}= \begin{cases}\tilde{\psi}_{*} \tilde{i} \tilde{\psi}_{*}^{-1} & \text { on } \operatorname{Im}(\tilde{\psi}), \\ \Pi_{*}^{-1} J \Pi_{*} & \text { on } \tilde{M} \backslash \operatorname{Im}(\tilde{\psi}) .\end{cases}
$$

Note that since $\pi$ and $\psi$ are holomorphic diffeomorphisms near the boundary of their respective domains, $\Pi_{*}^{-1} J \Pi_{*}=\tilde{\psi}_{*} \tilde{i} \tilde{\psi}_{*}^{-1}$ on $\tilde{\psi}(1+2 \epsilon) \backslash \tilde{\psi}(1+\epsilon)$, and so $\tilde{J}$ is well defined. To see that $\tilde{\omega}$ tames (is compatible with) $\tilde{J}$, we first note that $\Pi$ is holomorphic for $x \in \tilde{M}-\mathcal{L}(1+\epsilon)$, and we recall that $\tilde{\omega}=\Pi^{*} \omega$ on this region. Therefore, if $\omega$ tames $J$, then for $v, w \in T_{x} M, \tilde{\omega}(v, \tilde{J} v)=$ $\lambda^{2} \omega\left(\Pi_{*} v, \Pi_{*} \tilde{J} v\right)=\lambda^{2} \omega\left(\Pi_{*} v, J \Pi_{*} v\right)>0$, so $\tilde{\omega}$ tames $\tilde{J}$ on this region. If, in addition, $\omega$ is compatible with $J$, we have

$$
\begin{aligned}
\tilde{\omega}(\tilde{J} v, \tilde{J} w) & =\Pi^{*} \omega(\tilde{J} v, \tilde{J} w) \\
& =\omega\left(\Pi_{*} \tilde{J} v, \Pi_{*} \tilde{J} w\right) \\
& =\omega\left(J \Pi_{*} v, J \Pi_{*} w\right) \\
& =\omega\left(\Pi_{*} v, \Pi_{*} w\right)=\Pi^{*} \omega(v, w),
\end{aligned}
$$

as desired.

For $x \in \mathcal{L}(1+\epsilon)$, we have that $\tilde{\omega}=\left(\tilde{\psi}^{-1}\right)^{*} \tilde{\tau}$. Since $\tilde{\tau}$ is compatible with $\tilde{i}$, the canonical complex structure on $\mathcal{L}$, and $\tilde{\psi}$ is holomorphic, we have that $\tilde{\omega}$ is compatible with $\tilde{J}$ on this region. Therefore, if $\omega$ tames (is compatible with) $J$ on $M$, then $\tilde{\omega}$ tames (is compatible with) $\tilde{J}$ on all of $\tilde{M}$.

Blowing up more than one point is done as above for each ball in the disjoint product $\psi: \coprod_{j=1}^{k}\left(B_{j}(r), \omega_{0}, B_{\mathbb{R}, j}(r)\right) \hookrightarrow(M, \omega, L)$. It follows immediately from the construction that $\tilde{\omega}$ is in the desired cohomology class.

Remark 2.10. When we want to emphasize the embedding $\psi$, we will refer to the symplectic blow-up constructed as above as the blow-up of $M$ relative to $\psi$.

In the following proposition we construct a real structure on the blow-up $\tilde{M}$ given a real symplectic manifold $M$ and a suitably symmetric embedding $\psi$ of a disjoint union of balls into $M$. 
Proposition 2.11. Let $(M, \omega, \phi)$ be a real symplectic manifold, let $J$ be an $\omega$-tame (compatible) almost complex structure on $M$ which satisfies $\phi_{*} J \phi_{*}=$ $-J$, and let

$$
\psi: \coprod_{j=1}^{k}\left(B_{j}(1+2 \epsilon), \lambda_{j}^{2} \omega_{0}, i\right) \hookrightarrow(M, \omega, J)
$$

be a symplectic and holomorphic embedding. Suppose $\phi$ and $\psi$ satisfy

(1) $\operatorname{Im}(\phi \circ \psi)=\operatorname{Im}(\psi)$,

(2) $\operatorname{Im}\left(\phi \circ \psi_{j}\right) \cap \operatorname{Im}\left(\psi_{j}\right)=\emptyset$ if $\operatorname{Im}\left(\psi_{j}\right) \cap L=\emptyset$, and

(3) $\psi_{j} \circ c=\phi \circ \psi_{j}$ if $\operatorname{Im}\left(\psi_{j}\right) \cap L \neq \emptyset$.

Then there exists a real symplectic manifold $(\tilde{M}, \tilde{\omega}, \tilde{\phi})$ and a smooth onto map $\Pi: \tilde{M} \rightarrow M$ which satisfies

(1) $\Pi$ is a diffeomorphism on $\Pi^{-1}\left(M \backslash \cup_{j} \psi_{j}(0)\right)$,

(2) $\Pi^{-1}\left(\psi_{j}(0)\right) \cong \mathbb{C} P^{n-1}$,

(3) $\Pi \circ \tilde{\phi}=\phi \circ \Pi$, and

(4) $\tilde{\omega}$ is in the cohomology class

$$
[\tilde{\omega}]=\left[\Pi^{*} \omega\right]-\sum_{j=1}^{k} \pi \lambda_{j}^{2} e_{j},
$$

where the $e_{j}$ are the Poincaré duals of the exceptional classes

$$
E_{j}=\left[\Pi^{-1}\left(\psi_{j}(0)\right)\right] \in H_{2}(\tilde{M} ; \mathbb{Z}) .
$$

Furthermore, the real structure $\tilde{\phi}$ and the almost complex structure $\tilde{J}$ in the blow-up $\tilde{M}$ satisfy $\tilde{\phi}_{*} \tilde{J}=-\tilde{J} \tilde{\phi}_{*}$, and, for every $j$ with $\psi_{j} \circ c=\phi \circ \psi_{j}$, we have $\phi_{*} E_{j}=-E_{j} \in H_{2}(\tilde{M} ; \mathbb{Z})$.

Remark 2.12. As we will see in the proof, in the case where there are balls which are embedded off of the Lagrangian, the blow-up is not constructed relative to $\psi$, but relative to another symplectic, holomorphic embedding with the same image. The ball embeddings whose image intersects the Lagrangian are left untouched, and those which take pairs of balls to $M \backslash L$ are changed to commute with $\phi$ and the standard real structure on $\mathbb{R}^{2 n}$.

In order to prove this proposition, we use the following lemmas. In the first lemma, we construct the blow-up given a real embedding $\psi$ on one ball 
such that $\psi \circ c=\phi \circ \psi$. In the second, we construct the simultaneous blowup of an embedding $\psi$ of two balls $B_{1}$ and $B_{2}$ such that $\phi \circ \psi\left(B_{1}\right)=\psi\left(B_{2}\right)$.

Lemma 2.13. Let $(M, \omega, \phi)$ be a real symplectic manifold, let $J$ be an $\omega$ tame (compatible) almost complex structure on $M$ which satisfies $\phi_{*} J \phi_{*}=$ $-J$. Suppose the map

$$
\psi:\left(B(1+2 \epsilon), \lambda^{2} \omega_{0}, i\right) \hookrightarrow(M, \omega, J)
$$

is a symplectic and holomorphic embedding such that $\psi \circ c=\phi \circ \psi$. Then there exists a symplectic manifold $(\tilde{M}, \tilde{\omega})$ that admits an anti-symplectic involution $\tilde{\phi}$ such that $\Pi$ and $\tilde{\omega}$ satisfy the conclusions of Proposition 2.8 .

Furthermore, the real structure $\tilde{\phi}$ in the blow-up $\tilde{M}$ satisfies $\tilde{\phi}_{*} \tilde{J}=-\tilde{J} \tilde{\phi}_{*}$, and

$$
\tilde{\phi}_{*}\left[\Pi^{-1}(\psi(0))\right]=-\left[\Pi^{-1}(\psi(0))\right] \in H_{2}(\tilde{M} ; \mathbb{Z}) .
$$

Proof. We first note that $\psi$ is a relative embedding, since

$$
\psi^{-1}(\operatorname{Fix}(\phi))=\operatorname{Fix}(c)=B_{\mathbb{R}}(1+2 \epsilon) .
$$

Now construct the blow-up $(\tilde{M}, \tilde{\omega})$ of $(M, \omega)$ relative to $\psi$ as in Proposition 2.8. Denote by $\tilde{c}$ the complex conjugation map on $\mathcal{L}$ and recall that we have $\pi \circ \tilde{c}(z, l)=c \circ \pi(z, l)$, since $z \in l \Longleftrightarrow \bar{z} \in \bar{l}$ and $\overline{0}=0$. Given $\epsilon, \lambda>0$, let $\tilde{\tau}(\epsilon, \lambda)$ be the symplectic form on $\mathcal{L}$ constructed in Proposition 2.1, and recall that $\tilde{c}^{*} \tilde{\tau}(\epsilon, \lambda)=-\tilde{\tau}(\epsilon, \lambda)$. We now define a map $\tilde{\phi}: \tilde{M} \rightarrow \tilde{M}$ by

$$
\tilde{\phi}(x)= \begin{cases}\Pi^{-1} \circ \phi \circ \Pi(x), & x \in \tilde{M} \backslash \tilde{\psi}(\mathcal{L}(1+\epsilon)), \\ \tilde{\psi} \circ \tilde{c} \circ \tilde{\psi}^{-1}(x), & x \in \tilde{\psi}(\mathcal{L}(1+2 \epsilon)) .\end{cases}
$$

By the commutativity of Figure 2.3 and the equivariance of $\psi$, we have, for $x \in \mathcal{L}(1+2 \epsilon) \backslash \mathcal{L}(1+\epsilon)$,

$$
\begin{aligned}
\tilde{\psi} \circ \tilde{c} \circ \tilde{\psi}^{-1}(x) & =\tilde{\psi} \circ \pi^{-1} \circ c \circ \pi \circ \tilde{\psi}^{-1}(x) \\
& =\Pi^{-1} \circ \psi \circ c \circ \psi^{-1} \circ \Pi(x) \\
& =\Pi^{-1} \circ \psi \circ \psi^{-1} \circ \phi \circ \Pi(x)=\Pi^{-1} \circ \phi \circ \Pi(x) .
\end{aligned}
$$

Therefore $\tilde{\phi}$ is well defined and a diffeomorphism. Since $\Pi^{-1} \circ \phi \circ \Pi$ and $\tilde{\psi} \circ \tilde{c} \circ \tilde{\psi}^{-1}$ are anti-symplectic involutions on their respective domains, it follows that $\tilde{\phi}$ is an anti-symplectic involution. 
To see the last statement in the proposition, for $x \in \tilde{M} \backslash \tilde{\psi}(\mathcal{L}(1+\epsilon))$, we compute

$$
\begin{aligned}
\tilde{\phi}_{*} \tilde{J} & =\Pi_{*}^{-1} \phi_{*} \Pi_{*} \tilde{J} \\
& =-\Pi_{*}^{-1} J \phi_{*} \Pi_{*} \\
& =-\tilde{J} \Pi_{*}^{-1} \phi_{*} \Pi_{*}=-\tilde{J} \tilde{\phi} .
\end{aligned}
$$

For $x \in \tilde{\psi}(\mathcal{L}(1+2 \epsilon))$, we have

$$
\begin{aligned}
\tilde{\phi}_{*} \tilde{J} & =\tilde{\psi}_{*} \tilde{c}_{*} \tilde{\psi}_{*}^{-1} \tilde{J} \\
& =-\tilde{\psi}_{*} \tilde{i} \tilde{c}_{*} \tilde{\psi}^{-1} \\
& =-\tilde{J} \tilde{\psi}_{*} \tilde{c}_{*} \tilde{\psi}^{-1}=-\tilde{J} \tilde{\phi}
\end{aligned}
$$

as desired.

Let $E=\tilde{\psi}(\mathcal{L}(0))$. To see that $\tilde{\phi}_{*} E=-E$, we note that $\tilde{c}(\mathcal{L}(0))=\mathcal{L}(0)$, and that $\tilde{c}$ reverses orientation. This completes the proof.

Lemma 2.14. Let $(M, \omega, \phi)$ be a real symplectic manifold, and let $J$ be an $\omega$-tame (compatible) almost complex structure. Suppose

$$
\gamma: \coprod_{k=0}^{1}\left(B_{k}(1+2 \epsilon), \lambda^{2} \omega_{0}, i\right) \rightarrow(M, \omega, J)
$$

is a symplectic and holomorphic embedding such that $\operatorname{Im}\left(\phi \circ \gamma_{1}\right)=\operatorname{Im}\left(\gamma_{2}\right)$. Then there exist a real symplectic manifold $(\tilde{M}, \tilde{\omega})$ with real structure $\tilde{\phi}$, an $\tilde{\omega}$-tame (compatible) almost complex structure $J$, and a smooth onto map $\Pi: \tilde{M} \rightarrow M$ which satisfies the conclusions of Proposition 2.8 .

Furthermore, the real structure $\tilde{\phi}$ and the almost complex structure $\tilde{J}$ in the blow-up $\tilde{M}$ satisfy $\tilde{\phi}_{*} \tilde{J}=-\tilde{J} \tilde{\phi}_{*}$.

Proof. Define a map $\psi: \Pi_{k=0}^{1}\left(B_{k}(1+2 \epsilon), \lambda^{2} \omega_{0}, i\right) \rightarrow(M, \omega, J)$ by

$$
\psi(x)= \begin{cases}\gamma(x), & x \in B_{0}, \\ \phi \circ \gamma \circ c \circ \iota(x), & x \in B_{1},\end{cases}
$$

where $\iota: \Pi_{k=0}^{1} B_{k} \rightarrow \Pi_{k=0}^{1} B_{k}$ is the map given by $\iota\left(x \in B_{k}\right)=x \in B_{k+1} \bmod 2$. We note that, since $c$ and $\phi$ are anti-holomorphic and $\gamma$ is holomorphic, $\psi$ is holomorphic, and, similarly, since $c$ and $\phi$ and anti-symplectic, and $\gamma$ is symplectic, $\psi$ is symplectic as well. Furthermore, $\gamma, c, \phi$, and $\iota$ are all $1-1$, and 
we conclude that $\psi$ is a symplectic, holomorphic embedding. Now observe that $c \circ \iota$ is an antisymplectic involution on $\prod_{k=0}^{1} B_{k}, \operatorname{Im}(\psi)=\operatorname{Im}(\gamma)$ by definition, and that $\psi \circ c \circ \iota=\phi \circ \psi$, so that $\psi$ is a real embedding for the real structures $c \circ \iota$ and $\phi$. We now construct the blow-up of $M$ relative to $\psi$ as in McDuff and Polterovich [20] (which is as in the relative blow-up without the Lagrangian).

On $\coprod_{k=0}^{1} \mathcal{L}_{k}$, we put the anti-symplectic involution $\tilde{c} \circ \tilde{\iota}$, where $\tilde{c}$ is complex conjugation on $\mathcal{L}$, and, as above, $\tilde{\iota}: \coprod_{k=0}^{1} \mathcal{L}_{k} \rightarrow \coprod_{k=0}^{1} \mathcal{L}_{k}$ is given by $\tilde{\iota}\left((z, l) \in \mathcal{L}_{k}\right)=(z, l) \in \mathcal{L}_{k+1}$ mod 2 . Recall that $\pi \circ \tilde{c}(z, l)=c \circ \pi(z, l)$, since $z \in l \Longleftrightarrow \bar{z} \in \bar{l}$ and $\overline{0}=0$, and note that, by definition of $\iota$ and $\tilde{\iota}$, we also have $\pi \circ \tilde{c} \circ \tilde{\iota}(z, l)=c \circ \iota \circ \pi(z, l)$.

Given $\epsilon, \lambda>0$, we define $\nu(\epsilon, \lambda)$ to be the symplectic form on $\coprod_{k=0}^{1} \mathcal{L}_{k}$ $(1+2 \epsilon)$, such that the restriction on each $\mathcal{L}_{k}$ is given by $\left.\nu(\epsilon, \lambda)\right|_{\mathcal{L}_{k}}:=\tilde{\tau}(\epsilon, \lambda)$, where $\tilde{\tau}(\epsilon, \lambda)$ is the symplectic form on $\mathcal{L}$ constructed in Proposition 2.1.

Now define a map $\tilde{\phi}: \tilde{M} \rightarrow \tilde{M}$ by

$$
\tilde{\phi}(x)= \begin{cases}\Pi^{-1} \circ \phi \circ \Pi(x), & x \in \tilde{M} \backslash \tilde{\psi}\left(\coprod_{k=0}^{1} \mathcal{L}_{k}(1+\epsilon)\right) \\ \tilde{\psi} \circ \tilde{c} \circ \tilde{\iota} \circ \tilde{\psi}^{-1}(x), & x \in \tilde{\psi}\left(\coprod_{k=0}^{1} \mathcal{L}_{k}(1+2 \epsilon)\right),\end{cases}
$$

where $\tilde{\psi}$ is the embedding of $\coprod_{k=0}^{1} \mathcal{L}_{k}(1+2 \epsilon)$ as in Figure 2.3. By the commutativity of Figure 2.3, we have, for $x \in \mathcal{L}(1+2 \epsilon) \backslash \mathcal{L}(1+\epsilon)$

$$
\begin{aligned}
\tilde{\psi} \circ \tilde{c} \circ \tilde{\iota} \circ \tilde{\psi}^{-1}(x) & =\tilde{\psi} \circ \pi^{-1} \circ c \circ \iota \circ \pi \circ \tilde{\psi}^{-1}(x) \\
& =\Pi^{-1} \circ \psi \circ c \circ \iota \circ \psi^{-1} \circ \Pi(x) \\
& =\Pi^{-1} \circ \psi \circ \psi^{-1} \circ \phi \circ \Pi(x)=\Pi^{-1} \circ \phi \circ \Pi(x) .
\end{aligned}
$$

Therefore $\tilde{\phi}$ is well defined and a diffeomorphism. That $\tilde{\phi}$ is an antisymplectic involution follows from the fact that $\Pi^{-1} \circ \phi \circ \Pi$ and $\tilde{\psi} \circ \tilde{c} \circ \tilde{\iota} \circ$ $\tilde{\psi}^{-1}$ are anti-symplectic involutions on their respective domains.

To see the last statement in the proposition, for $x \in \tilde{M} \backslash \tilde{\psi}\left(\coprod_{k=0}^{1} \mathcal{L}_{k}\right.$ $(1+\epsilon))$, we compute

$$
\begin{aligned}
\tilde{\phi}_{*} \tilde{J} & =\Pi_{*}^{-1} \phi_{*} \Pi_{*} \tilde{J} \\
& =\Pi_{*}^{-1} \phi_{*} J \Pi_{*} \\
& =-\Pi_{*}^{-1} J \phi_{*} \Pi_{*} \\
& =-\tilde{J} \Pi_{*}^{-1} \phi_{*} \Pi_{*}=-\tilde{J} \tilde{\phi}_{*} .
\end{aligned}
$$


For $x \in \tilde{\psi}\left(\coprod_{k=0}^{1} \mathcal{L}_{k}(1+2 \epsilon)\right)$, we have

$$
\begin{aligned}
\tilde{\phi}_{*} \tilde{J} & =\tilde{\psi}_{*} \tilde{c}_{*} \tilde{\iota}_{*} \tilde{\psi}_{*}^{-1} \tilde{J} \\
& =\tilde{\psi}_{*} \tilde{c}_{*} \tilde{\iota}_{*} \tilde{i} \tilde{\psi}_{*}^{-1} \\
& =-\tilde{\psi}_{*} \tilde{i}_{\tilde{c}_{*}} \tilde{\iota}_{*} \tilde{\psi}_{*}^{-1} \\
& =-\tilde{J} \tilde{\psi}_{*} \tilde{c}_{*} \tilde{\iota}_{*} \tilde{\psi}_{*}^{-1}=-\tilde{J} \tilde{\phi}_{*},
\end{aligned}
$$

as desired.

Proof of Proposition 2.11. For each $\gamma_{i}$ with $\operatorname{Im}\left(\gamma_{i}\right) \cap L \neq \emptyset$ we construct the blow-up using Lemma 2.13. For each $\gamma_{i}$ such that $\operatorname{Im}\left(\gamma_{i}\right) \cap \operatorname{Fix}(\phi)=\emptyset$, we first recall that, by hypothesis, $\operatorname{Im}\left(\gamma_{i}\right) \cap \operatorname{Im}\left(\phi \circ \gamma_{i}\right)=\emptyset$. Since $\operatorname{Im}(\phi \circ \gamma)=$ $\operatorname{Im}(\gamma)$, then there is a $\gamma_{i^{\prime}}$ with $\operatorname{Im}\left(\phi \circ \gamma_{i}\right)=\operatorname{Im}\left(\gamma_{i^{\prime}}\right)$. We blow-up the pair $\gamma_{i}, \gamma_{i^{\prime}}$ using Lemma 2.14. The result follows.

We now remove the hypothesis that our ball embeddings are holomorphic. To do so, we isotope our form to a cohomologous one that admits a small holomorphic embedding around the center of our embedding, which we may do under appropriate assumptions on an almost complex structure that tames the symplectic form. We then create a family of symplectic forms $\tilde{\omega}_{t}$ on the blow-up such that the original one tames (or is compatible with) the almost complex structure $\tilde{J}$ on the blow-up, and the last one is in the cohomology class corresponding to the ball embedding. This is the same strategy used in McDuff and Polterovich [20], and the following proposition and its proof are variants of Proposition 2.1.C in [20], which we modify to keep track of the Lagrangians $L$ and $\tilde{L}$ throughout the process.

Proposition 2.15. (1) Let $\psi:\left(B(1+2 \epsilon), \lambda^{2} \omega_{0}, B_{\mathbb{R}}(1+2 \epsilon)\right) \rightarrow(M, \omega, L)$ be a relative symplectic embedding. Suppose that $J$ is an almost complex structure on $M$ which tames (is compatible with) $\omega$ and which is relatively integrable at $\psi(0)$.

Then there exists a manifold $\tilde{M}$ with a submanifold $\tilde{L}$, a family of symplectic forms $\tilde{\omega}_{t}, t \in[0,1]$ on $\tilde{M}$, an almost complex structure $\tilde{J}$ on $\tilde{M}$, and a smooth onto map $\Pi: \tilde{M} \rightarrow M$ such that $\tilde{\omega}_{0}$ tames (is compatible with) $\tilde{J}, \tilde{L}$ is a Lagrangian for all the $\tilde{\omega}_{t}, \Pi(\tilde{L})=L$, and $\tilde{\omega}_{1}$ satisfies

$$
\left[\tilde{\omega}_{1}\right]=\left[\Pi^{*} \omega\right]-\pi \lambda^{2} e,
$$

where $e$ is the Poincare dual of the class $\left[\Pi^{-1}\left(\psi^{\prime}(0)\right)\right] \in H_{2}(M ; \mathbb{Z})$.

(2) Suppose, furthermore, $M$ is a real symplectic manifold with real structure $\phi, \operatorname{Fix}(\phi)=\mathrm{L}, J$ satisfies $\phi_{*} J \phi_{*}=-J$, and $\psi \circ c=\phi \circ \psi$. Then 
there exists a family of real structures $\tilde{\phi}_{t}$ on $\tilde{M}$ such that $\tilde{\phi}_{t}^{*} \tilde{\omega}_{t}=-\tilde{\omega}_{t}$, $\left(\tilde{\phi}_{t}\right)_{*} \tilde{J}\left(\tilde{\phi}_{t}\right)_{*}=-\tilde{J}$.

The proof depends on the following proposition, which is an adaptation of Proposition 5.5.A in McDuff and Polterovich [20], and which we prove in Section 2.4.

Proposition 2.16. (1) Let $(M, \omega)$ be a symplectic manifold, and let $L \hookrightarrow$ $M$ be a Lagrangian submanifold. Let

$$
\psi:\left(B(1+2 \epsilon), \lambda^{2} \omega_{0}, B_{\mathbb{R}}(1+2 \epsilon)\right) \hookrightarrow(M, \omega, L)
$$

be a relative symplectic embedding, and let $J$ be an almost complex structure on $M$ which tames $\omega$ and is relatively integrable at $\psi(0) \in L$.

Then, for every compact subset $K \subset M \backslash \psi(0)$ there exists a symplectic form $\omega^{\prime}$ on $M$ isotopic to $\omega$ such that $\omega=\omega^{\prime}$ on $K$ and $\omega^{\prime}$ is $J$-standard in a neighborhood $\mathcal{N}$ of $\psi(0)$, i.e., $\omega^{\prime}$ is Kahler on $\mathcal{N}$, and the associated metric is flat in a neighborhood of $\psi(0)$.

In addition to the above, suppose that $M$ is a real symplectic manifold with real structure $\phi, \operatorname{Fix}(\phi)=L, J$ satisfies $\phi_{*} J \phi_{*}=-J, J$ is symmetrically integrable around $\psi(0)$, and $\phi \circ \psi=\psi \circ c$.

Then we can construct the symplectic form $\omega^{\prime}$ on $M$ to satisfy the conclusions above, and so that $\phi$ is a real structure for $\omega^{\prime}$ and $\omega$ and $\omega^{\prime}$ are isotopic through real symplectic forms.

Proof of Proposition 2.15. By Proposition 2.16, there is a symplectic form $\omega^{\prime}$ on $M$ which is isotopic to $\omega$ an $J$-standard. By Proposition 2.30, there is a diffeomorphism $F: M \rightarrow M$ such that $F^{*} \omega=\omega^{\prime}$. Replace $\omega$ by $\omega^{\prime}$ and $\psi$ by $F \circ \psi$. Abusing notation, we will refer to the new form and new embedding by $\omega$ and $\psi$, respectively. Since $\omega$ is now $J$ standard, it follows that, for some $\delta>0$, there exists a relative holomorphic symplectomorphism $\eta: B(\delta) \rightarrow$ $M, \eta(0)=\psi(0)$. Now define the function $S_{t}: B(1+2 \epsilon) \rightarrow B(1+2 \epsilon)$ by

$$
S_{t}(x)=\beta(t) x+(1-\beta(t))\left[\lambda(1+2 \epsilon) \delta^{-1} \alpha(|x|)+(1-\alpha(|x|))\right] x,
$$

where $\beta(t)$ is a bump function with $\beta(t)=1$ for $t \leq 0$ and $\beta(t)=0$ for $t \geq 1$, and $\alpha(t)$ is a bump function with $\alpha(t)=1$ for $t \leq \delta$ and $\alpha(t)=0$ for $t \geq 1+2 \epsilon-\epsilon^{\prime}$ for some small $\epsilon^{\prime}>0$. We wish to show that $S_{t}$ has the following properties:

(1) $S_{0}=\mathrm{Id}$, 
(2) $S_{t}$ is equal to the identity near $\partial B(1+2 \epsilon)$,

(3) $S_{t}^{*} \omega_{0}=\mu(t) \omega_{0}$, where $\mu(t): \mathbb{R} \rightarrow \mathbb{R}$ and $\mu(1)=\lambda^{2}(1+2 \epsilon)^{2} \delta^{-2}$ on $B(\delta)$ for some $\delta>0$,

(4) $c \circ S_{t}=S_{t} \circ c$, where $c$ denotes complex conjugation, and

(5) $B_{\mathbb{R}}(\lambda+\epsilon)$ is a Lagrangian for $S_{t}^{*} \omega_{0}$.

The first four items above follow directly from the definitions of $S_{t}, \alpha$, and $\beta$, and item (5) follows immediately from item (4).

Now let $F_{t}: M \rightarrow M$ be the extension of $\psi \circ S_{t} \circ \psi^{-1}: \operatorname{Im}(\psi) \subset M \rightarrow M$ by the identity map, and set $\omega_{t}=F_{t}^{*} \omega$. Define

$$
\nu_{t}(z):=\eta\left(\frac{\delta}{1+2 \epsilon} z\right):\left(B(1+2 \epsilon), \frac{\delta^{2}}{(1+2 \epsilon)^{2}} \mu(t) \omega_{0}\right) \rightarrow\left(M, \omega_{t}\right) .
$$

Since $\psi$ is relative holomorphic embedding, $\nu$ is also a holomorphic embedding, and since $\nu_{t}^{*} \omega_{t}=\frac{\delta^{2}}{(1+2 \epsilon)^{2}} \mu(t) \omega_{0}, \nu_{t}$ embeds symplectically into $\left(M, \omega_{t}\right)$ for every $t$. Now take the forms $\tilde{\omega}_{t}$ obtained by blowing up the family $\omega_{t}$ by the embeddings $\nu_{t}$. We claim that $\tilde{\omega}_{t}$ verifies the conclusion of the theorem. By definition, the $\nu_{t}$ are symplectic and holomorphic maps into $M$, and by hypothesis, $\omega_{0}$ is compatible with $J$, so by Proposition $2.8, \tilde{\omega}_{0}$ is compatible with $\tilde{J}$. Since $F_{1}$ is isotopic to the identity, we see that $\left[\omega_{1}\right]=[\omega]$, from which it follows that $\left[\Pi^{*} \omega_{1}\right]=\left[\Pi^{*} \omega\right] . \tilde{\omega}_{1}$ is therefore in the desired cohomology class, and the first part of the theorem is proved.

If $M$ has a real structure $\phi$, and $\psi$ satisfies the hypotheses in the latter half of the theorem, then since $c \circ S_{t}=S_{t} \circ c$, it follows that $\phi \circ F_{t}=F_{t} \circ \phi$, and therefore $\phi^{*} \omega_{t}=-\omega_{t}$. Blowing up $\left(M, \omega_{t}\right)$, we create a family of involutions $\tilde{\phi}_{t}: \tilde{M} \rightarrow \tilde{M}$ such that $\tilde{\phi}^{*} \tilde{\omega}_{t}=-\tilde{\omega}_{t}$ and $(\tilde{\phi})_{*} \tilde{J}(\tilde{\phi})_{*}=-\tilde{J}$, finishing the proof of the proposition.

We now prove Theorem 1.21.

Proof of Theorem 1.21. By Remark 1.20, there exists an almost complex structure $J$ on $M$ which is relatively integrable in a neighborhood of the points $\psi_{j}(0)$. Then by Proposition 2.15 , there exists a manifold $\tilde{M}$ with submanifold $\tilde{L}$ and a family of symplectic forms $\tilde{\omega}_{t}$ on $\tilde{M}$ such that $\tilde{L}$ is a Lagrangian for all $\tilde{\omega}_{t}$, and which satisfies $\left[\tilde{\omega}_{1}\right]=\left[\Pi^{*} \omega\right]-\sum_{k=1}^{q} \lambda_{k}^{2} e_{k}$, where the $e_{k}$ are the Poincaré duals of the exceptional spheres $C_{k}$ added in the blow-up.

If, in addition, $M$ has a real structure $\phi$ and $\operatorname{Im}(\psi)=\operatorname{Im}(\phi \circ \psi)$, then, by Remark $1.20, J$ may be chosen so that it is symmetrically integrable around the points $\psi_{j}(0)$ and $\phi_{*} J \phi_{*}=-J$. Therefore, by Proposition 2.15, 
there exists a family of maps $\tilde{\phi}_{t}$ on the blow-up such that $\tilde{\phi}_{t}^{*} \tilde{\omega}_{t}=-\tilde{\omega}_{t}$, and this proves the theorem.

\subsection{Blow-down}

We now construct the blow-down of a symplectic manifold $(\tilde{M}, \tilde{\omega}, \tilde{L})$. In particular, we will prove Theorem 1.22, stated again below.

Theorem (Theorem 1.22). (1) Let $(\tilde{M}, \tilde{\omega})$ be a symplectic manifold with Lagrangian $\tilde{L}$. Suppose there is a $(p, q)$-mixed symplectic embedding

$$
\tilde{\psi}: \coprod_{j=1}^{k}\left(\mathcal{L}_{j}\left(r_{j}\right), \rho_{j}\left(\delta_{j}, \lambda_{j}\right), \mathcal{R}_{j}\left(r_{j}\right)\right) \hookrightarrow(\tilde{M}, \tilde{\omega}, \tilde{L})
$$

such that $\psi^{-1}(\tilde{L})=\coprod_{j=1}^{p} \mathcal{R}_{j}\left(r_{j}\right)$. Let $C_{j} \subset \tilde{M}$ denote $\tilde{\psi}_{j}(\mathcal{L}(0))$, and let $C=\cup_{j} C_{j}$.

Then there exists a symplectic manifold $(M, \omega), a(p, q)$-mixed symplectic embedding

$$
\psi: \coprod_{j=1}^{k}\left(B(1+2 \epsilon), \lambda_{j} \omega_{0}, B_{\mathbb{R}}(1+2 \epsilon)\right) \rightarrow(M, \omega, L),
$$

a Lagrangian submanifold $L \subset M$, and a smooth onto map $\Pi: \tilde{M} \rightarrow$ $M$ such that the following is satisfied:

(a) $\Pi$ is a diffeomorphism on $\tilde{M} \backslash C$,

(b) $\Pi\left(C_{j}\right)=p_{j} \in M$, where $p_{j}$ is a point,

(c) $\Pi(\tilde{L})=L$, and

(d) $\omega$ satisfies

$$
[\tilde{\omega}]-\left[\Pi^{*} \omega\right] \in \mathcal{E}
$$

where $\mathcal{E}$ is the linear vector space generated by $e_{1}, \ldots, e_{k}$, the Poincaré duals of the exceptional classes $E_{j}=\left[\tilde{\psi}_{j}(0)\right]$.

(2) Suppose, in addition, $\tilde{M}$ admits an anti-symplectic involution $\tilde{\phi}$ which satisfies
(a) $\operatorname{Fix}(\tilde{\phi})=\tilde{L}$,
(b) $\operatorname{Im}(\tilde{\psi})=\operatorname{Im}(\tilde{\phi} \circ \tilde{\psi})$,
(c) $\operatorname{Im}\left(\tilde{\phi} \circ \tilde{\psi}_{i}\right) \cap \operatorname{Im}\left(\tilde{\psi}_{i}\right)=\emptyset$ if $\operatorname{Im}\left(\psi_{i}\right) \cap L=\emptyset$, and
(d) $\tilde{\psi}_{i} \circ \tilde{c}=\tilde{\phi} \circ \tilde{\psi}_{i}$ if $\operatorname{Im}\left(\tilde{\psi}_{i}\right) \cap \tilde{L} \neq \emptyset$.

Then $(M, \omega)$ admits an anti-symplectic involution $\phi$ such that $\phi \circ$ $\Pi=\Pi \circ \tilde{\phi}$. 
In parallel to the blow-up construction, we begin by constructing a family of forms on $\mathbb{C}^{n}$ from the forms $\rho(\delta, \lambda)$, which we will then use to construct the global form in the blow-down. The following proposition is adapted from Proposition 5.1.B in [20].

Proposition 2.17. For every $\epsilon, \delta, \lambda>0$, there exists a Kähler form $\tau=$ $\tau(\epsilon, \delta, \lambda)$ on $\mathbb{C}^{n}$ such that the following holds:

(1) $\pi^{*}(\tau)=\rho(\delta, \lambda)$ on $\mathcal{L}-\mathcal{L}(1+\epsilon)$,

(2) $\tau=\lambda^{2} \omega_{0}$ on $B(1) \subset \mathbb{C}^{n}$,

(3) $\tau$ is compatible with $i$,

(4) $c^{*} \tau=-\tau$, where $c$ denotes complex conjugation on $\mathbb{C}^{n}$,

(5) $\mathbb{R}^{n}$ is a Lagrangian for $\tau$.

Proof. Note first that $\rho(\delta, \lambda)=\delta^{2} \rho(1, \nu)$ for $\nu=\lambda / \delta$. Define

$$
h_{\lambda}(z):=\left(1+\left(\frac{\lambda}{|z|}\right)^{2}\right)^{1 / 2}
$$

and let $\epsilon_{0}>0$ be such that $2 \epsilon_{0}\left(\nu^{2}-1\right)+\epsilon_{0}^{2}\left(\nu^{2}-1\right)<1$. Let $\beta(t)$ be a smooth non-increasing function which is 1 for $t \leq 1$ and 0 for $t \geq 1+\epsilon_{0}$, and let $\gamma(t)$ be a smooth non-increasing function which is 1 for $t \leq 1+\epsilon_{0}$ and 0 for $t \geq 1+\epsilon$. Then we define the map $G: \mathbb{C}^{n} \rightarrow \mathbb{C}^{n}$ by

$$
G(z)= \begin{cases}\nu z & \text { for }|z| \leq 1 \\ \beta(|z|) \nu z+(1-\beta(|z|)) \nu\left(1+\epsilon_{0}\right) & \text { for } 1<|z|<1+\epsilon_{0}, \\ \gamma(|z|) \nu\left(1+\epsilon_{0}\right)+\left(1-\beta(|z|) h_{\nu}(z) z\right. & \text { for } 1+\epsilon_{0} \leq|z| \leq 1+\epsilon, \\ h_{\nu}(z) z & \text { for } 1+\epsilon<|z|\end{cases}
$$

and we define the form $\tau=\delta^{2} G^{*} \omega_{0}$. We claim that $\tau$ satisfies the properties in the proposition. First, to see the $G$ is a diffeomorphism, note that $\beta$ and $\gamma$ are smooth. Since $G$ is a diffeomorphism on each region, it now follows that $G$ is a diffeomorphism.

To see that $G$ is monotone, we let $t:=|z|$ and $H(t):=|G(z)|$. On the first region, it is clear from the definition of $G$ that $G$ is monotone. On the second region, we have

$$
\frac{d H}{d t}=\beta^{\prime}(t) \nu t+\beta(t) \nu-\beta^{\prime}(t)\left(1+\epsilon_{0}\right) \nu .
$$


The second term is always non-negative, and, since $t \leq 1+\epsilon_{0}$ and $\beta^{\prime}(t) \leq 0$, it follows that $\beta^{\prime}(t)\left(t-1-\epsilon_{0}\right) \geq 0$, and therefore $G$ is monotone on this region.

For the third region, we have

$$
\frac{d H}{d t}=\gamma^{\prime}(t)\left(1+\epsilon_{0}\right) \nu+\left(t^{2}+\nu^{2}\right) t(1-\beta(t))-\gamma^{\prime}(t)\left(t^{2}+\nu^{2}\right)^{1 / 2} .
$$

The second term is always non-negative, and since $2 \epsilon_{0}\left(\nu^{2}-1\right)+\epsilon_{0}^{2}\left(\nu^{2}-\right.$ 1) $<1$ by hypothesis, it follows that

$$
\nu^{2}+2 \nu^{2} \epsilon_{0}-2 \epsilon_{0}-\epsilon_{0}^{2}+\nu^{2} \epsilon_{0}^{2} \leq 1+\nu^{2} \Longrightarrow \nu^{2}\left(1+\epsilon_{0}\right)^{2} \leq \nu^{2}+\left(1+\epsilon_{0}\right)^{2} .
$$

Recall that $\gamma^{\prime}(t) \leq 0$, so taking the square root of both sides, we see that

$$
\gamma^{\prime}(t)\left(\nu^{2}\left(1+\epsilon_{0}\right)^{2}-\nu^{2}+\left(1+\epsilon_{0}\right)^{2}\right) \geq 0,
$$

and therefore $G$ is monotone on this region. Since $G$ is monotone on the last region by definition, $G$ is therefore monotone everywhere.

The first property in the conclusion of the lemma follows from Lemma 2.3 , the second from the definitions of $\tau$ and $G$ for $|z| \leq 1$, and the third follows from Lemmas 2.4 and 2.5 and the fact that $G$ is a monotone radial function. To see the fourth point, note that $G(z)=\alpha(|z|) z$ for some real-valued function $\alpha: \mathbb{R} \rightarrow \mathbb{R}$. This implies that $c \circ G=G \circ c$, where $c$ is complex conjugation on $\mathbb{C}^{n}$, and therefore $c^{*} \delta^{2} G^{*} \omega_{0}=\delta^{2} G^{*} c^{*} \omega_{0}=-\delta^{2} G^{*} \omega_{0}$, as desired. This, in turn, proves the fifth point as well, and completes the proof.

In parallel to the blow-up construction, we split the blow-down into two parts, the relative blow-down, in which we consider only a Lagrangian, and we do not consider a real structure, and the real blow-down. We now construct the relative blow-down.

Proposition 2.18. Let $(\tilde{M}, \tilde{\omega})$ be a symplectic manifold with Lagrangian $\tilde{L}$, and let $\tilde{J}$ be an $\tilde{\omega}$-tame (compatible) almost complex structure. Suppose there is a $(p, q)$-mixed holomorphic and symplectic embedding

$$
\psi: \coprod_{j=1}^{k}\left(\mathcal{L}_{j}\left(r_{j}\right), \rho_{j}\left(\delta_{j}, \lambda_{j}\right), \mathcal{R}_{j}\left(r_{j}\right), i\right) \hookrightarrow(\tilde{M}, \tilde{\omega}, \tilde{L}, \tilde{J})
$$

such that

$$
\psi^{-1}(L)=\coprod_{j=1}^{p} \mathcal{R}_{j}\left(r_{j}\right)
$$


Then the conclusions of the first part of Theorem 1.22 are satisfied.

Proof. We consider the case when $(p, q)=(1,0)$. Let

$$
\tilde{\psi}:\left(\mathcal{L}\left(1+2 \epsilon_{0}\right), \rho(\delta, \lambda), \mathcal{R}\left(1+2 \epsilon_{0}\right)\right) \rightarrow(\tilde{M}, \tilde{\omega}, \tilde{L})
$$

be a relative symplectic embedding such that $\tilde{\psi}^{*} \tilde{\omega}=\rho(\delta, \lambda)$. We then perform a local complex blow-down in $\mathcal{L}(1+2 \epsilon)$, and we define the manifold $M$ by

$$
M:=\tilde{M} \backslash \tilde{\psi}(\mathcal{L}(1+2 \epsilon)) \cup_{\tilde{\psi} \circ \pi^{-1} \mid \partial \mathcal{L}(1+2 \epsilon)} B(1+2 \epsilon)
$$

after which, as in the blow-up, we arrive at the commutative diagram

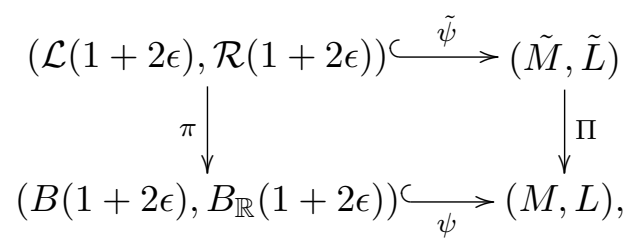

where $\Pi$ is defined by

$$
\Pi(x)= \begin{cases}x, & x \in \tilde{M} \backslash \tilde{\psi}(\mathcal{L}(1+2 \epsilon)), \\ \psi \circ \pi \circ\left(\tilde{\psi}^{-1}\right), & x \in \tilde{\psi}(\mathcal{L}(1+2 \epsilon)) .\end{cases}
$$

We now define the following form on $M$ :

$$
\omega= \begin{cases}\left(\Pi^{-1}\right)^{*} \tilde{\omega} & \text { on } M \backslash \psi(B(1+\epsilon)), \\ \left(\psi^{-1}\right)^{*} \tau(\epsilon, \delta, \lambda) & \text { on } \psi(B(1+2 \epsilon)) .\end{cases}
$$

We check that the definition of $\omega$ agrees on $\psi(B(1+2 \epsilon)) \backslash \psi(B(1+\epsilon))$. On this region, we have

$$
\begin{aligned}
\omega & =\left(\psi^{-1}\right)^{*} \tau(\epsilon, \delta, \lambda) \\
& =\left(\psi^{-1}\right)^{*}\left(\pi^{-1}\right)^{*} \rho(1, \lambda) \\
& =\left(\psi^{-1}\right)^{*} \pi^{*} \tilde{\psi}^{*} \tilde{\omega}=\left(\Pi^{-1}\right)^{*} \tilde{\omega},
\end{aligned}
$$

so $\omega$ is well defined. Furthermore, we claim that $\omega$ is a symplectic form. Too see this, note that $\Pi$ is a diffeomorphism on $\Pi^{-1}(M \backslash \psi(B(1+\epsilon)))$, so $\omega^{n}$ is a volume form on $M \backslash \psi(B(1+\epsilon))$, and $\omega$ is therefore non-degenerate there. It is closed by definition. For $\psi(B(1+2 \epsilon))$, we first note that by Proposition 2.17, $\tau$ is Kähler, and therefore symplectic on $\mathbb{R}^{2 n}$. Since $\psi^{-1}$ is 
a diffeomorphism on $B(1+2 \epsilon)$, $\omega$ is non-degenerate here as well, and closed by definition.

We define the almost complex structure $J$ on $M$ by

$$
J= \begin{cases}\psi_{*} i \psi_{*}^{-1} & \text { on } \operatorname{Im}(\psi), \\ \Pi_{*} \tilde{J} \Pi_{*}^{-1} & \text { on } M \backslash \operatorname{Im}(\psi) .\end{cases}
$$

Note that since $\pi$ and $\psi$ are holomorphic diffeomorphisms near the boundary of their respective domains, $\psi_{*} i \psi_{*}^{-1}=\Pi_{*} \tilde{J} \Pi_{*}^{-1}$ on $\psi(1+2 \epsilon) \backslash \psi$ $(1+\epsilon)$, and so $J$ is well defined. To see that $\omega$ tames (is compatible with) $J$, we first note that $\Pi$ is holomorphic and a diffeomorphism for $x \in \tilde{M}-\mathcal{L}(1+$ $\epsilon)$, and we recall that $\omega=\left(\Pi^{-1}\right)^{*} \tilde{\omega}$ on $M \backslash B(1+\epsilon)$. Therefore, if $\tilde{\omega}$ tames $J$, then for $v, w \in T_{\Pi(x)} M, \omega(v, J v)=\tilde{\omega}\left(\Pi_{*}^{-1} v, \Pi_{*}^{-1} \tilde{J} v\right)=\tilde{\omega}\left(\Pi_{*}^{-1} v, J \Pi_{*}^{-1} v\right)>$ 0 , so $\omega$ tames $J$ on $M \backslash \psi(B(1+\epsilon)$. If, in addition, $\tilde{\omega}$ is compatible with $\tilde{J}$, then on $M \backslash B(1+\epsilon)$, we have

$$
\begin{aligned}
\omega(J v, J w) & =\left(\Pi^{-1}\right)^{*} \tilde{\omega}(J v, J w) \\
& =\tilde{\omega}\left(\Pi_{*}^{-1} J v, \Pi_{*}^{-1} J w\right) \\
& =\tilde{\omega}\left(\tilde{J} \Pi_{*}^{-1} v, \tilde{J} \Pi_{*}^{-1} w\right)=\tilde{\omega}\left(\Pi_{*}^{-1} v, \Pi_{*}^{-1} w\right)=\left(\Pi^{-1}\right)^{*} \tilde{\omega}(v, w),
\end{aligned}
$$

as desired.

For $x \in \mathcal{L}(1+\epsilon)$, we have that $\omega=\left(\psi^{-1}\right)^{*} \tau$. Since $\tau$ is compatible with $i$, the canonical complex structure on $B(1+2 \epsilon)$, and $\psi$ is holomorphic (tautologically, by the definition of $J$ ), then $\omega$ is compatible with $J$ on this region. Therefore, if $\tilde{\omega}$ tames (is compatible with) $\tilde{J}$ on $\tilde{M}$, then $\omega$ tames (is compatible with) $J$ on $M$.

The condition on the cohomology class of $\omega$ follows immediately from the construction. This completes the proof of the proposition.

We now construct the real blow-down for a real symplectic manifold $\tilde{M}$.

Proposition 2.19. Let $(\tilde{M}, \tilde{\omega}, \tilde{\phi})$ be a real symplectic manifold and let $\tilde{L}=$ $\operatorname{Fix}(\tilde{\phi})$. Let $\tilde{J}$ be an $\tilde{\omega}$-tame (compatible) almost complex structure on $\tilde{M}$. Suppose that

$$
\tilde{\psi}: \coprod_{j=1}^{k}\left(\mathcal{L}_{j}\left(r_{j}\right), \rho_{j}\left(\delta_{j}, \lambda_{j}\right), \mathcal{R}_{j}\left(r_{j}\right), i\right) \hookrightarrow(\tilde{M}, \tilde{\omega}, \tilde{L}, \tilde{J})
$$

is a symplectic and holomorphic embedding such that

(1) $\psi^{-1}(\tilde{L})=\coprod_{j=1}^{k} \mathcal{R}_{j}\left(r_{j}\right)$, 
(2) $\operatorname{Im}(\tilde{\psi})=\operatorname{Im}(\tilde{\phi} \circ \tilde{\psi})$,

(3) $\operatorname{Im}\left(\tilde{\phi} \circ \tilde{\psi}_{j}\right) \cap \operatorname{Im}\left(\tilde{\psi}_{i}\right)=\emptyset$ if $\operatorname{Im}\left(\psi_{j}\right) \cap L=\emptyset$, and

(4) $\tilde{\psi}_{j} \circ \tilde{c}=\tilde{\phi} \circ \tilde{\psi}_{j}$ if $\operatorname{Im}\left(\tilde{\psi}_{j}\right) \cap \tilde{L} \neq \emptyset$.

Then the conclusions of the second part of Theorem 1.22 are satisfied.

As in the blow-up, we prove this in two parts. The first is the following.

Lemma 2.20. Let $(\tilde{M}, \tilde{\omega}, \tilde{\phi})$ be a real symplectic manifold and let $\tilde{L}=$ $\operatorname{Fix}(\tilde{\phi})$. Let $\tilde{J}$ be an $\tilde{\omega}$-tame (compatible) almost complex structure on $\tilde{M}$, and suppose that $\tilde{\psi}:(\mathcal{L}(r), \rho(\delta, \lambda), \mathcal{R}(r)) \hookrightarrow(\tilde{M}, \tilde{\omega}, \tilde{L})$ is a symplectic embedding such that $\tilde{\psi} \circ c=\tilde{\phi} \circ \tilde{\psi}$. Then the blow-down $(M, \omega, L)$ admits an antisymplectic involution $\phi$ and an almost complex structure $J$ such that $\operatorname{Fix}(\phi)=L$ and $\phi_{*} J \phi_{*}=-J$.

Proof. Construct the blow-down $(M, \omega)$ as in Proposition 2.18. Now define a map $\phi$ by

$$
\phi(x)= \begin{cases}\Pi \circ \tilde{\phi} \circ \Pi^{-1}, & x \in M \backslash \psi(B(1+\epsilon)), \\ \psi \circ c \circ \psi^{-1}(x), & x \in \psi(B(1+2 \epsilon)) .\end{cases}
$$

Note that, for $x \in \psi(B(1+2 \epsilon)-B(1+\epsilon))$,

$$
\begin{aligned}
\psi \circ c \circ \psi^{-1}(x) & =\psi \circ \pi \circ \tilde{c} \circ \pi^{-1} \circ \psi^{-1}(x) \\
& =\Pi \circ \tilde{\psi} \circ \tilde{c} \circ \tilde{\psi}^{-1} \circ \Pi^{-1}(x) \\
& =\Pi \circ \tilde{\phi} \circ \Pi^{-1}(x),
\end{aligned}
$$

so the map $\phi$ is well defined and a diffeomorphism. Furthermore, $\phi^{2}=$ Id by definition. To see that $\phi^{*} \omega=-\omega$, we have, for $x \in M \backslash \psi(B(1+2 \epsilon))$,

$$
\begin{aligned}
\phi^{*} \omega_{x} & =\phi^{*}\left(\Pi^{-1}\right)^{*} \tilde{\omega}_{x} \\
& =\left(\Pi^{-1}\right)^{*} \tilde{\phi}^{*} \tilde{\omega}_{x} \\
& =-\left(\Pi^{-1}\right) \tilde{\omega}_{x}=-\omega_{x}
\end{aligned}
$$

and for $x \in \psi(B(1+2 \epsilon))$, we have

$$
\begin{aligned}
\phi^{*} \omega_{x} & =\left(\psi^{-1}\right)^{*} c^{*} \psi^{*}\left(\psi^{-1}\right)^{*} \tau(\epsilon, \delta, \lambda) \\
& =\left(\psi^{-1}\right)^{*} c^{*} \tau(\epsilon, \delta, \lambda) \\
& =-\left(\psi^{-1}\right)^{*} \tau(\epsilon, \delta, \lambda)=-\omega_{x}
\end{aligned}
$$


We now check that $\phi_{*} J \phi_{*}=-J$. For $x \in M \backslash \psi(B(1+\epsilon))$, we compute

$$
\begin{aligned}
\phi_{*} J & =\Pi_{*} \tilde{\phi}_{*} \Pi_{*}^{-1} J \\
& =\Pi_{*} \tilde{\phi}_{*} \tilde{J} \Pi_{*}^{-1} \\
& =-\Pi_{*} \tilde{J} \tilde{\phi}_{*} \Pi_{*}^{-1} \\
& =-J \Pi_{*}^{-1} \phi_{*} \Pi_{*}=-J \phi_{*} .
\end{aligned}
$$

For $x \in \psi(B(1+2 \epsilon))$, we have

$$
\begin{aligned}
\phi_{*} J & =\psi_{*} c_{*} \psi_{*}^{-1} J \\
& =\psi_{*} c_{*} i \psi_{*}^{-1} \\
& =-\psi_{*} i c_{*} \psi_{*}^{-1} \\
& =-J \psi_{*} c_{*} \psi_{*}^{-1}=-J \phi_{*},
\end{aligned}
$$

which completes the proof.

Lemma 2.21. Let $(\tilde{M}, \tilde{\omega}, \tilde{\phi})$ be a real symplectic manifold and let $\tilde{L}=$ $\operatorname{Fix}(\tilde{\phi})$. Suppose that $\tilde{\gamma}: \coprod_{j=0}^{1}\left(\mathcal{L}_{j}\left(r_{j}\right), \rho_{j}\left(\delta_{j}, \lambda_{j}\right), \mathcal{R}(r)\right) \hookrightarrow(\tilde{M}, \tilde{\omega}, \tilde{L})$ is a symplectic embedding such that $\psi^{-1}(\tilde{L})=\emptyset$ and $\operatorname{Im}\left(\left.\tilde{\phi} \circ \tilde{\gamma}\right|_{\mathcal{L}_{0}}\right)=\operatorname{Im}\left(\left.\tilde{\gamma}\right|_{\mathcal{L}_{1}}\right)$. Then the blow-down $(M, \omega)$ admits an anti-symplectic involution $\phi$.

Proof. Since $\operatorname{Im}\left(\left.\tilde{\phi} \circ \tilde{\gamma}\right|_{\mathcal{L}_{0}}\right)=\operatorname{Im}\left(\left.\tilde{\gamma}\right|_{\mathcal{L}_{1}}\right)$, we can replace $\gamma$ with an embedding

$$
\tilde{\psi}: \coprod_{j=0}^{1}\left(\mathcal{L}_{j}\left(r_{j}\right), \rho_{j}\left(\delta_{j}, \lambda_{j}\right), \mathcal{R}(r)\right) \hookrightarrow(\tilde{M}, \tilde{\omega}, \tilde{L})
$$

defined by

$$
\tilde{\psi}= \begin{cases}\tilde{\gamma}(x), & x \in \mathcal{L}_{0}, \\ \tilde{\phi} \circ \tilde{\gamma} \circ \tilde{c} \circ \tilde{\iota}(x), & x \in \mathcal{L}_{1},\end{cases}
$$

where $\tilde{\iota}: \coprod_{j=0}^{1} \mathcal{L}_{j} \rightarrow \coprod_{j=0}^{1} \mathcal{L}_{j}$ is given by $\tilde{\iota}\left(x \in \mathcal{L}_{j}\right)=x \in \mathcal{L}_{j+1} \bmod 2$. Note that $\tilde{c} \circ \tilde{\iota}$ is a real structure on $\coprod_{j=0}^{1} \mathcal{L}_{j}$ which makes $\tilde{\psi}$ a real map. The proof now follows exactly the proof of Lemma 2.20 , with $\tilde{c} \circ \tilde{\iota}$ in place of $\tilde{c}$.

Proof of Proposition 2.19. For each $\tilde{\psi}_{j}$ with $\operatorname{Im}\left(\tilde{\psi}_{j}\right) \cap L \neq \emptyset$, we construct the blow-down as in Lemma 2.20. The rest of the maps come in pairs by assumption, and for each pair, we construct the blow-down as in Lemma 2.21. The proposition follows. 
Theorem 1.22 now follows easily from the above propositions. We finish the proof here.

Proof of Theorem 1.22. First, by Remark 1.20, there is an $\epsilon^{\prime}>0, \epsilon^{\prime}<\epsilon$, and an $\tilde{\omega}$-tame almost complex structure $\tilde{J}$ such that $\tilde{J}$ is integrable on $\psi_{j}(\mathcal{L}(1+$ $\left.2 \epsilon^{\prime}\right)$ ) and which makes $\left.\psi\right|_{\mathcal{L}_{j}\left(1+2 \epsilon^{\prime}\right)}$ holomorphic. Define $\mathcal{N}:=\coprod_{j=1}^{k} \mathcal{L}_{j}(1+$ $\left.2 \epsilon^{\prime}\right)$. If $M$ is not a real manifold, then we use Proposition 2.18 to blow down $\tilde{M}$ using the map $\left.\psi\right|_{\mathcal{N}}$. For a real manifold $\tilde{M}$ and a real embedding $\tilde{\psi}$, the theorem then follows from Proposition 2.19, again using the restriction $\left.\psi\right|_{\mathcal{N}}$. This completes the proof.

Remark 2.22. We should note that the forms obtained in the local models, i.e., Propositions 2.1 and 2.17 are not the same as the forms constructed, respectively, from blowing up $\mathbb{C}^{n}$ at 0 and blowing down $\mathcal{L}$ along the exceptional divisor using Theorems 1.21 and 1.22. Constructing the genuine blow-up and blow-down forms, even of $\mathbb{C}^{n}$ and $\mathcal{L}$, still requires an auxiliary symplectic embedding of either $B(r)$ or $\mathcal{L}(r)$, and these are absent from the form constructions of $\tau$ and $\tilde{\tau}$ in Propositions 2.1 and 2.17. Because of this, we still use the constructions of Theorems 1.21 and 1.22, even in these cases.

\subsection{Invariant symplectic neighborhoods and the Moser stability theorem in real symplectic manifolds}

In this section we present a version of the Symplectic Neighborhood Theorem adapted to leave invariant the fixed-point set of a real symplectic manifold $(M, \omega, \phi)$. We will use this below to establish real packing results in $\left(\mathbb{C} P^{2}, \mathbb{R} P^{2}\right)$ and other real symplectic 4-manifolds. We closely follow the presentation of the analogous theorems for symplectic manifolds with no real structure in McDuff and Salamon [21].

We begin with a definition.

Definition 2.23. Let $M$ be a smooth manifold and let $G$ be a compact Lie group which acts smoothly on $M$. We say that a vector field $X$ on $M$ is equivariant with respect to $G$ (or $G$-equivariant) if $\forall x \in M, g \in G$, we have $X(g x)=g_{*} X(x)$.

We now give the following standard result in equivariant dynamics, which we quote from Ortega and Ratiu [24] (Proposition 3.3.2(i)). 
Proposition 2.24. Let $M$ be a smooth manifold, $A$ a subgroup of the group of diffeomorphisms of $M$. Let $U$ be an A-invariant open subset of $M$, and $X$ an A-equivariant vector field defined on $U$. Then, the domain of definition $\operatorname{Dom}\left(F_{t}\right) \subset U$ of the flow $F_{t}$ of $X$ is A-invariant and $F_{t}$ is itself A-equivariant.

Lemma 2.25. Let $(M, \omega, \phi)$ be a real symplectic manifold with $\operatorname{Fix}(\phi)=L$, and suppose $\omega_{t}, t \in[0,1]$ is a smooth family of symplectic forms with $\omega_{0}=\omega$ and $\phi^{*} \omega_{t}=-\omega_{t}$. Suppose, furthermore, that there exists a family of oneforms $\sigma_{t}$ with $\frac{d}{d t} \omega_{t}=d \sigma_{t}$ and $\phi^{*} \sigma_{t}=-\sigma_{t}$. Then there exists a family of diffeomorphisms $\alpha_{t}: M \rightarrow M$ such that

$$
\begin{aligned}
\alpha_{t}^{*} \omega_{t} & =\omega_{0}, \\
\alpha_{t}(L) & \subseteq L, \\
\alpha_{t} \circ \phi & =\phi \circ \alpha_{t} .
\end{aligned}
$$

Proof. We first note that, since the $\omega_{t}$ are non-degenerate, there exists a unique vector field $X_{t}$ which satisfies

$$
\sigma_{t}+\iota\left(X_{t}\right) \omega_{t}=0
$$

Given such a vector field $X_{t}$, let $\alpha_{t}$ be the solutions of

$$
\begin{aligned}
\frac{d}{d t} \alpha_{t} & =X_{t} \circ \alpha_{t}, \\
\alpha_{0} & =\mathrm{Id} .
\end{aligned}
$$

We now note that, because $\omega_{t}$ is closed, $d \omega_{t}=0$, and $\frac{d}{d t} \omega_{t}=d \sigma_{t}$, equation (2.8) implies that

$$
0=\alpha_{t}^{*}\left(\frac{d}{d t} \omega_{t}+\iota\left(X_{t}\right) d \omega_{t}+d \iota\left(X_{t}\right) \omega_{t}\right)=\frac{d}{d t} \alpha_{t}^{*} \omega_{t} .
$$

If $X_{t}$ is $\phi$-equivariant, then the flow $\alpha_{t}$ will be $\phi$-equivariant as well. To see that $X_{t}$ is $\phi$-equivariant, we first remark that

$$
\begin{aligned}
\phi^{*}\left(\sigma_{t}+\iota\left(X_{t}\right) \omega_{t}\right) & =0, \\
& =\phi^{*} \sigma_{t}+\phi^{*} \iota\left(X_{t}\right) \omega_{t} \\
& =-\sigma_{t}+\phi^{*} \iota\left(X_{t}\right) \omega_{t},
\end{aligned}
$$


which implies that $\phi^{*} \iota\left(X_{t}\right) \omega_{t}=\sigma_{t}=-\iota\left(X_{t}\right) \omega_{t}$. Therefore, for all $v \in T_{q} M$,

$$
\omega_{t}\left(\phi(q) ; X_{t}(\phi(q)), \phi_{*} v\right)=-\omega_{t}\left(q ; X_{t}(q), v\right) .
$$

However, $-\omega_{t}\left(q ; X_{t}(q), v\right)=\omega_{t}\left(\phi(q) ; \phi_{*} X_{t}(q), \phi_{*} v\right)$, so

$$
\omega_{t}\left(\phi(q) ; X_{t}(\phi(q)), \phi_{*} v\right)=\omega_{t}\left(\phi(q) ; \phi_{*} X_{t}(q), \phi_{*} v\right) .
$$

Since this is true for all $v \in T_{q} M, \phi_{*}$ is an isomorphism, and $\omega_{t}$ is nondegenerate, this implies that $\phi_{*} X_{t}(q)=X_{t}(\phi(q))$, and therefore the vector field $X_{t}$ is $\phi$-equivariant.

Furthermore, for $v \in T_{q} L, v \neq 0$, we have that $\sigma_{t}(q ; v)=-\sigma_{t}\left(q ; \phi_{*} v\right)=$ 0 , so $\omega\left(q ; X_{t}, v\right)=0$, which implies that $X_{t} \in T_{q} L \subset T_{q} M$. Since this is true for all $t \in[0,1]$, the diffeomorphisms $\alpha_{t}$ determined by equation (2.9) satisfy the constraints in equation (2.7) as required.

Lemma 2.26. Let $M$ be a $2 n$-dimensional smooth manifold, and let $\phi$ : $M \rightarrow M$ be a diffeomorphism with $\phi^{2}=\mathrm{Id}$. Let $L=\operatorname{Fix}(\phi)$, and suppose $Q \subset M$ is a $\phi$-invariant submanifold. Suppose that $\omega_{0}, \omega_{1} \in \Omega^{2}(M)$ are closed two forms with $\phi^{*} \omega_{i}=-\omega_{i}$ and such that, at every point $q \in Q$, $\left.\omega_{0}\right|_{T_{q} M}=\left.\omega_{1}\right|_{T_{q} M}$ and the $\omega_{i}$ are non-degenerate on $T_{q} M$. Then there exist neighborhoods $\mathcal{N}_{0}, \mathcal{N}_{1}$ of $Q$ and a diffeomorphism $\alpha: \mathcal{N}_{0} \rightarrow \mathcal{N}_{1}$ which satisfies

(1) $\left.\alpha\right|_{Q}=\mathrm{Id}$,

(2) $\alpha^{*} \omega_{1}=\omega_{0}$,

(3) $\alpha\left(\mathcal{N}_{0} \cap L\right) \subset L$,

(4) $\alpha \circ \phi=\phi \circ \alpha$.

Proof. We may assume that $Q \cap L \neq \emptyset$, since, if this was not the case, we could just take the $\mathcal{N}_{i}$ small enough so that $\mathcal{N}_{i} \cap L=\emptyset$ and invoke the ordinary symplectic neighborhood theorem.

Let $\mathcal{N}_{0}$ be a $\phi$-invariant tubular neighborhood of $L$. We first show that there exists a 1 -form $\sigma \in \Omega^{1}\left(\mathcal{N}_{0}\right)$ such that

$$
\begin{aligned}
\left.\sigma\right|_{T_{Q} M} & =0=\left.\sigma\right|_{T L}, \\
\phi^{*} \sigma & =-\sigma, \\
d \sigma & =\omega_{1}-\omega_{0} .
\end{aligned}
$$


To prove this, we endow $M$ with a $\phi$-invariant Riemannian metric, and consider the restriction of the exponential map to the normal bundle $T Q^{\perp}$. Since $Q$ is $\phi$-invariant, $T Q$ is $\phi_{*}$ invariant inside $T M$, and, therefore, since $\phi_{*}$ is an isomorphism from $T_{x} M$ to $T_{\phi(x)} M, T Q^{\perp}$ is $\phi_{*}$-invariant as well. Now, for a real number $\epsilon>0$, consider the neighborhood of the zero section of $T Q^{\perp}$

$$
V_{\epsilon}=\left\{(q, v) \in T M\left|q \in Q, v \in T_{q} Q^{\perp},\right| v \mid<\epsilon\right\} .
$$

Define the set $U_{\epsilon}:=\left(V_{\epsilon} \cup \phi\left(V_{\epsilon}\right)\right)$. Then $U_{\epsilon}$ is $\phi$-invariant, and for $\epsilon$ sufficiently small, the restriction of the exponential map to $U_{\epsilon}$ is a diffeomorphism from $U_{\epsilon}$ to a neighborhood $\mathcal{N}_{1}$ of $Q$. By a standard result in equivariant differential topology (Lemma 3.6 to be proven in Section 3.1), exp is equivariant as well. Now define $\psi_{t}: U_{\epsilon} \rightarrow \mathcal{N}_{1}, 0<t<1$, by $\psi_{t}(\exp (q, v))=\exp (q, t v)$. For $t>0, \psi_{t}$ is a diffeomorphism onto its image. At $t=0, \operatorname{Im}(\psi) \subseteq Q$, at $t=1, \psi_{1}=\mathrm{Id}$, and $\left.\psi_{t}\right|_{Q}=\mathrm{Id}$ for all $t \in[0,1]$. Since exp is equivariant, we also have $\psi_{t} \circ \phi(\exp (q, v))=\psi_{t}\left(\exp \left(c(q), \phi_{*} v\right)\right)=\exp \left(\phi(q), t \phi_{*} v\right)=$ $\phi \circ \exp (q, t v)=\phi \circ \psi_{t}$, so $\phi$ and $\psi_{t}$ commute.

Let $\tau=\omega_{1}-\omega_{0}$. Then $\psi_{0}^{*} \tau=0$ and $\psi_{1}^{*} \tau=\tau$, and, since $\psi_{t}$ is an equivariant diffeomorphism, we may define a $\phi$-equivariant vector field for $t>0$ by $X_{t}=\left(\frac{\partial}{\partial t} \psi_{t}\right) \circ \psi_{t}^{-1}$. Note that $X_{t}$ becomes singular at $t=0$. Nonetheless, we have

$$
\frac{d}{d t} \psi_{t}^{*} \tau=\psi_{t}^{*} \mathcal{L}_{X_{t}} \tau=d\left(\psi_{t}^{*} \iota\left(X_{t}\right) \tau\right)
$$

Let $\sigma_{t}=\psi_{t}^{*} \iota\left(X_{t}\right) \tau$. Therefore, $\frac{d}{d t} \psi_{t}^{*} \tau=d \sigma_{t}$, and, by the definition of $X_{t}, \sigma_{t}$ is equal to

$$
\sigma_{t}(q ; v)=\tau\left(\psi_{t}(q) ; \frac{d}{d t} \psi_{t}(q), d \psi_{t}(q) v\right) .
$$

Since $\sigma_{t}$ vanishes on $Q$ for all $t$, we may define $\sigma_{0}=0$, making $\sigma_{t}$ a smooth family for $t \in[0,1]$. In addition, we have that

$$
\tau=\psi_{1}^{*} \tau-\psi_{0}^{*} \tau=\int_{0}^{1} \frac{d}{d t} \psi_{t}^{*} \tau d t=d \sigma,
$$

where $\sigma=\int_{0}^{1} \sigma_{t} d t$. It also follows from the equivariance of $\psi_{t}$ that $(q, v) \in$ $T L, \sigma_{t}=0$ for all $t \in[0,1]$. To see this, note that for $(q, v) \in T L, d \psi_{t}(q) v \in$ $T_{q} L$, and since $\psi_{t}(q) \in L$ for all $t$, then $\frac{d}{d t} \psi_{t}(q) \in T_{\psi_{t}(q)} L$ as well, making $\sigma_{t}(q ; v)$ vanish by definition of $\tau$, because $L$ is Lagrangian for $\omega_{0}$ and $\omega_{1}$. To 
see that $\phi^{*} \sigma_{t}=-\sigma_{t}$, we compute

$$
\begin{aligned}
\phi^{*} \sigma_{t}(v)= & \phi^{*} \tau\left(\psi_{t}(q) ; \frac{d}{d t} \psi_{t}(q), d \psi_{t}(q) \cdot\right)(v) \\
= & \omega_{1}\left(\psi_{t} \circ \phi(q) ; \frac{d}{d t} \psi_{t}(\phi(q)), d \psi_{t} \circ d \phi(q) v\right) \\
& -\omega_{0}\left(\psi_{t} \circ \phi(q) ; \frac{d}{d t} \psi_{t}(\phi(q)), d \psi_{t} \circ d \phi(q) v\right) \\
= & \omega_{1}\left(\phi \circ \psi_{t}(q) ; \frac{d}{d t} \phi \circ \psi_{t}(q), d \phi \circ d \psi_{t}(q) v\right) \\
& -\omega_{0}\left(\phi \circ \psi_{t}(q) ; \frac{d}{d t} \phi \circ \psi_{t}(q), d \phi \circ d \psi_{t}(q) v\right) \\
= & \left(\omega_{1}\left(\phi \circ \psi_{t}(q) ; d \phi \frac{d}{d t} \psi_{t}(q), d \phi \circ d \psi_{t}(q) v\right)\right. \\
& \left.-\omega_{0}\left(\phi \circ \psi_{t}(q) ; d \phi \frac{d}{d t} \psi_{t}(q), d \phi \circ d \psi_{t}(q) \cdot v\right)\right) \\
= & -\tau\left(\psi_{t}(q) ; \frac{d}{d t} \psi_{t}(q), d \psi_{t}(q) v\right) \\
= & -\sigma_{t}(v) .
\end{aligned}
$$

Therefore, $\phi^{*} \sigma=\int_{0}^{1} \phi^{*} \sigma_{t} d t=-\omega$. We have now created the desired 1-form.

Now consider the family of 2 -forms on $\mathcal{N}_{0}$ given by $\omega_{t}=\omega_{0}+t\left(\omega_{1}-\right.$ $\left.\omega_{0}\right)=\omega_{0}+t d \sigma, t \in[0,1]$, and note that $\phi^{*} \omega_{t}=-\omega_{t}$ and $\frac{d}{d t} \omega_{t}=d \sigma$. The result now follows from Lemma 2.25.

Theorem 2.27. For $j=0,1$ let $\left(M_{j}, \omega_{j}, c_{j}\right)$ be real symplectic manifolds with compact $c_{j}$-invariant symplectic submanifolds $Q_{j}$. Suppose that there is an equivariant symplectic isomorphism $\Phi: \nu_{Q_{0}} \rightarrow \nu_{Q_{1}}$ of the symplectic normal bundles to $Q_{0}$ and $Q_{1}$ such that the restriction of $\Phi$ to the zero section is the symplectomorphism $\psi:\left(Q_{0}, \omega_{0}\right) \rightarrow\left(Q_{1}, \omega_{1}\right)$. Then there exist $c_{j}$-invariant neighborhoods $\mathcal{N}_{j}$ of the $Q_{j}$ such that $\psi$ extends to an equivariant symplectomorphism $\psi^{\prime}:\left(\mathcal{N}_{0}, \omega_{0}, c_{0}\right) \rightarrow\left(\mathcal{N}_{1}, \omega_{1}, c_{1}\right)$, and $d \psi^{\prime}$ induces $\Phi$ on $\nu_{Q_{0}}$.

Proof. We first show that $\psi$ extends to an equivariant diffeomorphism

$$
\psi_{1}: \mathcal{N}\left(Q_{0}\right) \rightarrow \mathcal{N}\left(Q_{1}\right)
$$


that induces the map $\Phi$ on $\nu_{Q_{0}}$. By Lemma 3.6, we may take the maps $\exp _{i}$ on $T M_{i}$ to be equivariant with respect to $c_{i}$. Define the map $\psi_{1}=$ $\exp _{1} \circ \Phi \circ \exp _{0}^{-1}$, and consider the forms $\omega_{0}$ and $\omega_{1}{ }^{\prime}=\left(\psi_{1}\right)^{*} \omega_{1}$ on $\mathcal{N}\left(Q_{0}\right)$. Note that, by construction, they are non-degenerate and they correspond on $T_{Q_{0}} M_{0}$. By Lemma 2.26, there is an equivariant diffeomorphism $\bar{\psi}$ of $\mathcal{N}\left(Q_{0}\right)$ such that $\bar{\psi}^{*} \omega_{1}{ }^{\prime}=\omega_{0}$. The composition $\psi^{\prime}=\psi_{1} \circ \bar{\psi}$ is the desired map.

Proposition 2.28. Let $(M, \omega, \phi)$ be a real symplectic manifold with real locus $L:=\operatorname{Fix}(\phi)$. Let $x \in L$. Then there exists a symplectic equivariant map from a neighborhood $\mathcal{U}$ of 0 in $\left(\mathbb{R}^{2 n}, \omega_{0}, c\right)$ to a neighborhood $\mathcal{V}$ of $x \in M$.

In order to prove this proposition, we will need the following lemma.

Lemma 2.29. Let $\Phi: \mathbb{R}^{2 n} \rightarrow \mathbb{R}^{2 n}$ be a linear map such that $\Phi^{2}=\mathrm{Id}$ and $\Phi^{*} \omega_{0}(v, w)=-\omega_{0}(v, w)$ for all $v, w \in \mathbb{R}^{2 n}$. Then there exists a linear symplectic isomorphism $\Psi: \mathbb{R}^{2 n} \rightarrow \mathbb{R}^{2 n}$ such that $\Psi \Phi=c_{*} \Psi$, where $c$ is the standard anti-symplectic involution on $\mathbb{R}^{2 n}$.

Proof. We first consider the case $n=1$. (We do this to demonstrate the construction. The proof does not proceed by induction.) Let $v \in \operatorname{Fix}(\Psi)=$ $\operatorname{Fix}\left(c_{*}\right)$ such that $\omega_{0}(v, i v)=1$, where $i$ is the standard complex structure on $\mathbb{R}^{2}$. Then $\mathbb{R}^{2}=\operatorname{Span}\{v, i v\}$. Let $w$ be an eigenvector of $\Psi$ with eigenvalue -1 . Let $\beta:=\omega_{0}(v, w)$. Now note that $\{v, i v\}$ and $\{v, w\}$ are bases for $\mathbb{R}^{2}$. We define the map $\Psi: \mathbb{R}^{2} \rightarrow \mathbb{R}^{2}$ to be the matrix sending $v \mapsto v$ and $w \mapsto\left(0, \omega_{0}(v, w)\right)$, where the coordinates are the standard $(x, y)=(v, i v)$ coordinates on $\mathbb{R}^{2}$. Then, for two vectors $a v+b w, c v+d w$, we have

$$
\begin{aligned}
\omega_{0}(a v+b w, c v+d w) & =\omega_{0}(a v, d w)+\omega_{0}(b w, c v) \\
& =(a d-b c) \beta
\end{aligned}
$$

On the other hand,

$$
\begin{aligned}
\omega_{0}(\Psi(a v+b w), \Psi(c v+d w)) & =\omega_{0}(a v+\beta \cdot b i v, c v+\beta \cdot d i v) \\
& =(a d-b c) \beta .
\end{aligned}
$$

Since the constants $a, b, c, d \in \mathbb{R}$ were arbitrary, we see that $\Psi$ is a linear symplectomorphism. Without loss of generality, consider a linear anti-symplectic involution $\Phi: \mathbb{R}^{2 n} \rightarrow \mathbb{R}^{2 n}$ with $\operatorname{Fix}(\Phi)=\mathbb{R}^{n}$. Let $e_{i}, i \in\{1, \ldots, 2 n\}$ denote the standard basis in $\mathbb{R}^{2 n}$, and consider the standard coordinates $\left(x_{1}, \ldots, x_{n}\right.$, $\left.y_{1}, \ldots, y_{n}\right)$ in $\mathbb{R}^{2 n}$. Take a basis $\left(v_{1}, \ldots, v_{n}\right)$ of the -1 eigenspace of $\Phi$, and 
define the map $\Psi: \mathbb{R}^{2 n} \rightarrow \mathbb{R}^{2 n}$ to be the unique linear map sending $e_{i} \mapsto e_{i}$, and $v_{i} \mapsto\left(0, \ldots, 0, \omega_{0}\left(e_{1}, v_{i}\right), \ldots, \omega_{0}\left(e_{n}, v_{i}\right)\right)$, where there are $n$ leading zeros in the coordinate (i.e., the -1 eigenspace of $\Phi$ is sent to the -1 eigenspace of $c_{*}$ ).

We now show that $\Psi$ is a symplectomorphism. First note that for $i \in$ $\{1, \ldots, n\}$ we have $\omega_{0}\left(e_{i}, e_{j}\right)=0=\Phi^{*} \omega_{0}\left(e_{i}, e_{j}\right)$. Furthermore, we see that

$$
-\omega_{0}\left(v_{i}, v_{j}\right)=\Phi^{*} \omega_{0}\left(v_{i}, v_{j}\right)=\omega_{0}\left(\Phi v_{i}, \Phi v_{j}\right)=\omega_{0}\left(v_{i}, v_{j}\right)
$$

which implies that $\omega_{0}\left(v_{i}, v_{j}\right)=0=\Phi^{*} \omega\left(v_{i}, v_{j}\right)$. Now note that

$$
\Phi^{*} \omega_{0}\left(e_{i}, v_{j}\right)=\omega_{0}\left(e_{i}, v_{j}\right) \omega_{0}\left(e_{i}, e_{i}\right)=\omega_{0}\left(e_{i}, v_{j}\right)
$$

as desired. Since $\Psi \Phi=\Phi c_{*}$, the proof of the lemma is complete.

Proof of 2.28. We first consider a $\phi$-invariant chart $(U, \alpha), \alpha: U \subset M \rightarrow \mathbb{R}^{2 n}$ centered at the point $p \in L$ which sends $L \rightarrow \mathbb{R}^{n} \subset \mathbb{C}^{n}$. We now consider the real structure $\Phi:=\alpha \circ \phi \circ \alpha^{-1}$ on $\operatorname{Im}(\alpha)$. By Lemma 2.29, there is a linear symplectic isomorphism $\Psi: \mathbb{R}^{2 n} \rightarrow \mathbb{R}^{2 n}$ such that $\Phi_{*} \Psi=c_{*} \Psi$ at the point 0 . Now apply Theorem 2.27 to the point $0 \in \mathbb{R}^{2 n}$.

We now prove a real version of the Moser stability theorem.

Proposition 2.30. Let $M$ be a closed manifold, and suppose that $\omega_{t}$ is a family of cohomologous symplectic forms on $M$ with anti-symplectic involution $\phi$, i.e., such that $\phi^{*} \omega_{t}=-\omega_{t}$. Then there is a family of diffeomorphisms $\psi_{t}$ such that $\phi \circ \psi_{t}=\psi_{t} \circ \phi, \psi_{0}=i d$, and $\psi_{t}^{*} \omega=\omega_{t}$.

Proof. We must show that there is a smooth family of one forms $\sigma_{t}$ such that

$$
d \sigma_{t}=\frac{d}{d t} \omega_{t}
$$

and $\phi^{*} \sigma_{t}=-\sigma_{t}$.

The proof of Moser stability theorem (Theorem 3.17 in [21]) shows that there exists a smooth family of one forms $\tau_{t}$ satisfying (2.10). Let $\sigma_{t}=\frac{1}{2}\left(\tau_{t}-\phi^{*} \tau_{t}\right)$. Then $d \sigma_{t}=\frac{1}{2}\left(\frac{d}{d t} \omega_{t}-\phi^{*} \frac{d}{d t} \omega_{t}\right)=\frac{1}{2}\left(\frac{d}{d t} \omega_{t}-\frac{d}{d t} \phi^{*} \omega_{t}\right)=\frac{d}{d t} \omega_{t}$. Applying Lemma 2.25, we arrive at the desired result. 


\subsection{Locally holomorphic maps}

In this section we prove Proposition 2.16, which shows that, given a relative or real symplectic embedding

$$
\psi:\left(B(1+2 \epsilon), \lambda^{2} \omega_{0}, B_{\mathbb{R}}(1+2 \epsilon)\right) \rightarrow(M, \omega, L)
$$

and an almost complex structure on $M$ which satisfies some additional conditions, we may find a form $\omega^{\prime}$ on $M$ isotopic to $\omega$, and a relative symplectic embedding $\psi^{\prime}:\left(B(\delta), \lambda^{2} \omega_{0}, B_{\mathbb{R}}(\delta)\right) \rightarrow\left(M, \omega^{\prime}, L\right)$ whose image is contained in the image of $\psi$ but which is holomorphic near the origin. We state the main proposition of this section here.

Proposition (Proposition 2.16). (1) Let $(M, \omega)$ be a symplectic manifold and let $L \subset M$ be a Lagrangian submanifold. Let

$$
\psi:\left(B(1+2 \epsilon), \lambda^{2} \omega_{0}, B_{\mathbb{R}}(1+2 \epsilon)\right) \rightarrow(M, \omega, L)
$$

be a relative symplectic embedding, and let $J$ be an almost complex structure on $M$ which tames $\omega$ and is relatively integrable at $\psi(0) \in L$.

Then, for every compact subset $K \subset M \backslash \psi(0)$ there exists a symplectic form $\omega^{\prime}$ on $M$ isotopic to $\omega$ such that $\omega=\omega^{\prime}$ on $K$ and $\omega^{\prime}$ is $J$-standard in a neighborhood $\mathcal{N}$ of $\psi(0)$, i.e., $\omega^{\prime}$ is Kahler on $\mathcal{N}$, and the associated metric is flat in a neighborhood of $\psi(0)$.

In addition to the above, suppose that $M$ is a real symplectic manifold with real structure $\phi, \operatorname{Fix}(\phi)=L, J$ satisfies $\phi_{*} J \phi_{*}=-J, J$ is symmetrically integrable around $\psi(0)$, and $\phi \circ \psi=\psi \circ c$.

Then we can construct the symplectic form $\omega^{\prime}$ on $M$ to satisfy the conclusions above, and so that $\phi$ is a real structure for $\omega^{\prime}$ and $\omega$ and $\omega^{\prime}$ are isotopic through real symplectic forms.

In the proof we will use the following lemma, which is a modification of Proposition 5.5.B in McDuff and Polterovich [20].

Lemma 2.31. Let $\omega$ be a symplectic form on $B(1)$ which tames the standard complex structure $i$ and satisfies $c^{*} \omega=-\omega$ for the standard real structure $c$. Then there exists a symplectic form on $B(1)$, say $\Omega$, with the following properties:

(1) $\Omega$ coincides with $\omega$ near the boundary of the ball;

(2) $\Omega$ tames $i$; 
(3) $\Omega$ is i-standard near 0, i.e., it is Kähler, and the associated metric is flat.

(4) $c^{*} \Omega=-\Omega$, and, in particular, $B_{\mathbb{R}}(1)$ is a Lagrangian for $\Omega$.

Proof. We divide the proof into three steps.

Step 1. We claim that for every $\kappa>1$ and every $1>\epsilon>0$, there exists a Kähler form, say $\tau_{\kappa}$, on $B(1)$ which is equal to $\kappa^{2} \omega_{0}$ in $B(\epsilon / 2 \kappa)$ and coincides with $\epsilon^{2} \omega_{0}$ near the boundary, where $\omega_{0}$ is the standard symplectic form on $B(1)$. Indeed, take a monotone map $h$ defined by $h(z)=(\kappa / \epsilon) z$ for $z \in B(\epsilon / 2 \kappa)$ and such that $h$ is equal to the identity map near the boundary. Then the form $\tau_{\kappa}=h^{*}\left(\epsilon^{2} \omega\right)$ is Kähler by Lemma 2.5.

Step 2. Let $\rho$ be a bump function on $\mathbb{R}^{2 n}$ which is radial, equal to 1 near the origin, and which vanishes for $|z|>1-\delta$, for some $\delta>0$. Let $\omega_{0}$ be the standard symplectic form on $\mathbb{R}^{2 n}$. Choose $\epsilon>0$ so that $\omega-\epsilon^{2} \omega_{0}$ tames $i$, and set $\rho_{\alpha}(z)=\rho((2 \alpha / \epsilon) z)$, with $1<\alpha<\kappa$. Finally, denote by $\beta$ a primitive of $\omega$ so that $\omega=d \beta$. Now consider the form

$$
\omega^{\prime}(\kappa)=\omega+\left(\tau_{\kappa}-\epsilon^{2} \omega_{0}-d\left(\rho_{\alpha} \beta\right)\right)
$$

We claim that $\omega^{\prime}(\kappa)$ satisfies the first four properties provided $\kappa$ is sufficiently large.

We note that $\omega^{\prime}(\kappa)$ coincides with $\omega$ near the boundary, and near the origin $\omega^{\prime}(\kappa)$ is equal to $\left(\kappa^{2}-\epsilon^{2}\right) \omega_{0}$, and is therefore $J$-standard there. Moreover, $\rho_{\alpha}=0$ outside $B((\epsilon / 2 \alpha)(1-\delta))$, and therefore $\omega^{\prime}(\kappa)=\omega-\left(\epsilon^{2} \omega_{0}+\right.$ $\left.\tau_{\kappa}\right)$ there. By assumption on $\epsilon, \omega-\epsilon^{2} \omega_{0}$ tames $i$ on this region, and since $\tau_{k}$ is Kähler, $\omega^{\prime}(\kappa)$ tames $i$ as well.

We now check that $\omega^{\prime}(\kappa)$ tames $i$ inside $B(\epsilon / 2 \alpha)$. On this region

$$
\omega^{\prime}(\kappa)=\left(\kappa^{2}-\epsilon^{2}\right) \omega_{0}+\left(1-\rho_{\alpha}\right) \omega-d \rho_{\alpha} \wedge \beta
$$

Let $|\cdot|$ denote the Euclidean distance of a vector in $\mathbb{R}^{2} n$. Since $\bar{B}(\epsilon / 2 \alpha)$ is compact, the sphere bundle

$$
S=\{(x, \xi)|x \in \bar{B},| \xi \mid=1\} \subset T \mathbb{R}^{2 n}
$$

is compact, and therefore the function $d \rho_{\alpha} \wedge \beta(\xi, i \xi)$ has a maximum, say $M$ on $S$. Therefore, for any $\xi \in T_{x}(B(\epsilon / 2 \alpha))$,

$$
d \rho_{\alpha} \wedge \beta(\xi, i \xi)=|\xi|^{2} d \rho_{\alpha} \wedge \beta\left(\frac{\xi}{|\xi|}, i \frac{\xi}{|\xi|}\right)
$$


and therefore the maximum of $d \rho \wedge \beta(\xi, i \xi)$ on $S_{a}=\{(x, \xi)|x \in \bar{B},| \xi \mid=$ $a\} \subset T \mathbb{R}^{2 n}$ is $|\xi|^{2} M$. Since $\omega$ tames $i$ and $1-\rho \geq 0,\left(1-\rho_{\alpha}\right) \omega(\xi, i \xi)>0$ for all $\xi \neq 0$, and we conclude that $\omega^{\prime}(\kappa)(\xi, i \xi)>\left(\kappa^{2}-\epsilon^{2}-M\right)|\xi|^{2}$. Since the quantity on the right is positive for sufficiently large $\kappa, \omega^{\prime}(\kappa)$ tames $i$ if we choose $\kappa$ large enough.

Step 3. We see from the above that the symplectic form $\omega^{\prime}(\kappa)$ satisfies the first three properties, but does not necessarily respect the real structure. By Lemma 4.4, however, since $\omega^{\prime}(\kappa)$ tames $i$ and $c_{*} i c_{*}=-i$, the form $\Omega=$ $\frac{1}{2}\left(\omega^{\prime}(\kappa)-c^{*} \omega^{\prime}(\kappa)\right)$ is symplectic, and satisfies the last property. We check that it satisfies the first three properties as well. $\Omega$ tames $i$ by Lemma 4.4, and, since $\omega^{\prime}(\kappa)=\left(\kappa^{2}-\epsilon^{2}\right) \omega_{0}$ near the origin, $\Omega(\kappa)=\omega^{\prime}(\kappa)$ near the origin, and is therefore $i$-standard on the same region as $\omega^{\prime}(\kappa)$. Furthermore, since $\omega^{\prime}(\kappa)$ coincides with $\omega$ near the boundary of the ball and $c^{*} \omega=-\omega$, then $\Omega=\omega^{\prime}(\kappa)=\omega$ near the boundary of the ball as well. Thus $\Omega(\kappa)$ satisfies the conclusion of the lemma for $\kappa$ sufficiently large, and this completes the proof.

Proof of Proposition 2.16. We first assume the hypotheses in Item 1 of the proposition, i.e., that $M$ is a real symplectic manifold with real structure $\phi$, $\operatorname{Fix}(\phi)=L, \psi \circ c=\phi \circ \psi$, and $J$ is a tame almost complex structure that is symmetrically integrable and satisfies $\phi_{*} J \phi_{*}=-J$. We split the proof into two steps.

Step 1. Let $(V, \gamma), \gamma: V \subset M \rightarrow \mathbb{C}^{n}$ be a symmetric, holomorphic chart centered at $\psi(0)$, which exists because $J$ is symmetrically integrable around $\psi(0)$. Let $W \subset \gamma(V)$ be a small ball centered at 0 inside $\gamma(V)$, and let $c$ denote complex conjugation on $W$. By Lemma 2.31, there exists a symplectic form $\bar{\omega}$ on $W$ which tames $i$, satisfies $c^{*} \bar{\omega}=-\bar{\omega}$, is $i$-standard near 0 , and coincides with $\left(\gamma^{-1}\right)^{*} \omega$ near the boundary of $W$. Let the form $\omega^{\prime}$ on $M$ be given by

$$
\omega_{x}^{\prime}= \begin{cases}\omega_{x} & \text { for } x \in M \backslash \gamma^{-1}(W), \\ \left(\gamma^{-1}\right)^{*} \bar{\omega} & \text { for } x \in \gamma^{-1}(W) .\end{cases}
$$

Note that since $\bar{\omega}$ tames $i$ on $W$ and $\gamma$ is symmetric and holomorphic, $\omega^{\prime}$ tames $J$ on $M, \omega^{\prime}$ is $J$-standard near $\psi(0)$, and $\phi^{*} \omega^{\prime}=-\omega^{\prime}$. Therefore, for each $s \in[0,1], \omega_{s}=\omega^{\prime}+s\left(\omega-\omega^{\prime}\right)$ is a symplectic form, and, furthermore, $\phi^{*} \omega_{s}=-\omega_{s}$ for all $s \in[0,1]$. Since the closed form $\omega-\omega^{\prime}$ is non-zero only on a contractible set, each of the $\omega_{s}$ are in the same cohomology class in $H^{2}(M ; \mathbb{R})$ and $\omega$ and $\omega^{\prime}$ are therefore isotopic through real symplectic forms.

For the first part of the theorem, we note that, since $J$ is relatively integrable, there is a chart $(V, \gamma)$ around $\psi(0)$ such that $\gamma(L) \subset \mathbb{R}^{n}$ and 
where $c \circ \gamma(V)=V$. If we use this chart in the place of $(V, \gamma)$ above and follow the same reasoning as above, the result follows.

\section{Topological criterion for the real blow-down}

In this section we prove Theorem 1.24, which gives a sufficient condition for blowing down a real Lagrangian submanifold.

\subsection{Equivariant differential topology}

In this subsection, we collect the statements of several classical results from equivariant differential topology which we will need for the proof of Theorem 1.24. The proofs are found in Bredon [5] and Kawakubo [12].

Definition 3.1. Let $M$ be a $C^{\infty}$ manifold, and $G$ be a compact Lie group. If $\Phi: G \times M \rightarrow M$ is a smooth action of $G$, then we call $\Phi$ a $G$-action on $M$, and if $M$ admits such a $G$-action, we call $M$ a $G$-manifold.

Lemma 3.2. Let $G$ be a compact Lie group, and let $M$ be a finitedimensional G-manifold. Then there exists a G-invariant Riemannian metric $g$ on $M$.

Lemma 3.3. Let $G$ be a compact Lie group, and let $M$ be a topological $G$-space. Then the fixed point set of $G, M^{G}$, is a closet set.

Theorem 3.4. Let $M$ be a $G$-manifold with $G$ finite. If $A$ is a closed $G$ invariant submanifold of $M$, then $A$ has an open $G$-invariant tubular neighborhood in $M$.

Proposition 3.5. Let $G$ be a compact Lie group, and let $M$ be a $G$ manifold. Then the fixed point set of $G, M^{G}$, is a smooth closed submanifold of $G$.

Lemma 3.6. Let $G$ be a compact Lie group, let $M$ be a finite-dimensional $G$-manifold and let $g$ be a G-invariant Riemannian metric. Then the associated exp map is G-equivariant.

\subsection{Proof of the blow-down criterion}

In this section, we prove Theorem 1.24, which we restate for convenience. 
Theorem (Theorem 1.24). Let $\left(M^{4}, \omega, \phi\right)$ be a real symplectic manifold with $L:=\operatorname{Fix}(\phi)$, and let $J$ be an almost complex structure on $M$ which tames $\omega$ and which satisfies $\phi_{*} J \phi_{*}=-J$. Suppose $E \in H_{2}(M ; \mathbb{Z})$ satisfies $E \cdot E=-1$ and $\phi_{*} E=-E$, and that there exists an embedded $J$ holomorphic curve $C$ which represents $E$. Then there exists a real symplectic manifold $(\check{M}, \check{\omega}, \check{\phi})$ and a smooth onto map $\Pi: M \rightarrow \check{M}$ that satisfies

(1) $\Pi$ is a diffeomorphism on $M \backslash C$,

(2) $\Pi(C)=p \in \check{M}$, where $p$ is a point,

(3) $\Pi \circ \phi=\check{\phi} \circ \Pi$, and

(4) $\check{\omega}$ satisfies

$$
[\omega]-\left[\Pi^{*} \check{\omega}\right] \in \mathcal{E},
$$

where $\mathcal{E}$ is the linear vector space generated by e, the Poincaré dual of the exceptional class $E=\left[\Pi^{-1}(p)\right]$.

We begin by recalling a version of the adjunction inequality, as given by Theorem 1.3 in McDuff [18].

Theorem 3.7. Let $(M, J)$ be an almost complex 4-manifold and $A \in$ $\mathrm{H}_{2}(M ; \mathbb{Z})$ be a homology class that is represented by a somewhere injective (closed) J-holomorphic curve $u: \Sigma \rightarrow M$. Then

$$
g \leq 1+\frac{1}{2}\left(A \cdot A-c_{1}(A)\right),
$$

with equality iff $u$ is an embedding, where $g$ is the genus of $\Sigma$.

We recall Definition 1.23 from Section 1.

Definition. We call $E \in H_{2}\left(M^{4} ; \mathbb{Z}\right)$ an exceptional class if $E \cdot E=-1$. If $u: \Sigma \hookrightarrow M^{4}$ is an embedding of the surface $\Sigma$, and $u_{*}[\Sigma]=E$, then we say that $u(\Sigma)$ is an exceptional curve.

It follows from the adjunction formula that

Corollary 3.8. Let $M$ be a 4-manifold, and let $u: \Sigma \hookrightarrow M$ be an exceptional 2 -sphere in $M$ such that $u_{*}[\Sigma]=E \in H_{2}(M ; \mathbb{Z})$. Then $c_{1}\left(u_{*}[\Sigma]\right)=1$.

Proof. Since $u$ is an embedding by definition, $0=1+\frac{1}{2}\left(E \cdot E-c_{1}(E)\right)$, and therefore $c_{1}(E)=1$. 
Remark 3.9. Suppose $(M, \omega, \phi)$ is a real symplectic manifold with an almost complex structure $J$ which tames $\omega$ and satisfies $\phi_{*} J \phi_{*}=-J$. Let $u: \Sigma \rightarrow M$ be a closed $J$-holomorphic curve, and suppose it is an embedding whose image is invariant under $\phi$. Then $\Sigma$ inherits the symplectic form $u^{*} \omega$ and the anti-symplectic involution $u^{-1} \circ \phi \circ u$.

Proposition 3.10. Let $\left(S^{2}, \omega\right)$ be endowed with an anti-symplectic involution $\phi$. If $\operatorname{Fix}(\phi) \neq \emptyset$, then the fixed point set of $\phi$ is a circle.

Proof. Let $G=\mathbb{Z}_{2}$ with smooth actions on $M$ given by the functions $\{\mathrm{Id}, \phi\}$. From Proposition 3.5 we see that $\operatorname{Fix}(G)=\operatorname{Fix}(\phi)$ is a closed submanifold of $S^{2}$. Denote this submanifold by $K$. Now suppose $p \in K$, and let $v, w \in T_{p} K$. Then $\omega(v, w)=\phi^{*} \omega(v, w)=-\omega(v, w)=0$, and so the fixed point set is an isotropic submanifold of $S^{2}$. By Remark 1.4, $L$ is Lagrangian, and therefore one dimensional. $\operatorname{Fix}(\phi)$ is therefore equal to a closed Lagrangian submanifold of $S^{2}$ and is therefore diffeomorphic to a union of non-intersecting circles. This union is compact, and therefore finite, since $\operatorname{Fix}(\phi)$ is topologically closed and $S^{2}$ is compact.

Suppose there is more than one circle in $\operatorname{Fix}(\phi)$, say $\alpha_{1}, \ldots, \alpha_{k}$. Now choose two circles, which we denote $\gamma_{1}$ and $\gamma_{2}$. $S^{2}$ therefore decomposes as $S^{2}=D_{1} \cup C \cup D_{2}$, where the $D_{i}$ are the non-intersecting disks bounded by the $\gamma_{i}$, and $C$ is the closed cylinder between the discs. Now consider $\phi\left(D_{1}\right)$. Since $\phi$ is a diffeomorphism, it must send $D_{1}$ onto a disc bounded by $\gamma_{1}$, i.e., either $D_{1}$ or $C \cup D_{2}$.

Now suppose $\phi\left(D_{1}\right)=C \cup D_{2}$. Then there is a point $x \in D_{1}$ such that $\phi(x) \in \gamma_{2} \Rightarrow \phi^{2}(x) \in \gamma_{2} \nsubseteq D_{1}$, which contradicts the assumption that $\phi^{2}=$ Id. Therefore, $\phi\left(D_{1}\right)=D_{1}$. Note that for any $x \in \gamma_{1}$, one of the eigenvalues of $d \phi(x)$ is -1 . Therefore, there are points in a collar neighborhood of $\gamma_{1}$ in $D_{1}$ which are sent by $\phi$ to a collar neighborhood of $\gamma_{1}$ in $D_{2} \cup C$. However, this contradicts that $\phi\left(D_{1}\right)=D_{1}$, and concludes the proof.

Corollary 3.11. Let $\left(M^{4}, \omega, \phi\right)$ be a real symplectic manifold, and let $L:=$ $\operatorname{Fix}(\phi)$. Let $J$ be an almost complex structure on $M$ such that $\phi_{*} J \phi_{*}=-J$, and let $E \in H_{2}(M ; \mathbb{Z})$ be an exceptional class with $\phi_{*} E=-E$. Suppose $u$ : $S^{2} \rightarrow M$ is an embedded rational J-holomorphic curve that represents $E$. Then $u(\Sigma) \cap L$ is diffeomorphic to a circle.

Proof. Note first that $\phi \circ u \circ c$ is another $J$-holomorphic embedding that represents $E$, and its image is equal to $\operatorname{Im}(\phi \circ u)$. Suppose now that $\operatorname{Im}(u) \neq$ $\operatorname{Im}(\phi \circ u)$. Let $c$ denote complex conjugation on $\Sigma=S^{2}$. Because both maps $u$ and $\phi \circ u \circ c$ are $J$-holomorphic, their intersections are at most countable, 
and since $[\operatorname{Im}(\phi \circ u \circ c)]=[\operatorname{Im}(u)]=E \in H_{2}(M ; \mathbb{Z})$, positivity of intersections in dimension 4 (e.g., Theorem E.1.4 in McDuff and Salamon [22]) implies that $0 \leq|\{\operatorname{Im} u\} \cap\{\operatorname{Im} \phi \circ u \circ c\}| \leq E \cdot E=-1$, which is a contradiction. Therefore, $\operatorname{Im}(u)=\operatorname{Im}(\phi \circ u)$. By Remark 3.9, $u(\Sigma)$ inherits a real structure from $M$, and it follows from Proposition 3.10 that the fixed point set of $\phi$ restricted to $u(\Sigma)$ is a circle. Since $\operatorname{Fix}(\phi)=L \subset M$, it follows that $u(\Sigma) \cap L$ is diffeomorphic to a circle.

Lemma 3.12. There is a natural isomorphism between the oriented Lagrangian subspaces of $\mathbb{R}^{2 n}$ and the quotient space $U(n) / S O(n)$.

Proof. We recall from McDuff and Salamon [21] that the unitary matrix $U=X+i Y$ given by a unitary Lagrangian frame is determined by the Lagrangian subspace $\Lambda$ up to right multiplication by a matrix in $O(n)$. Similarly, given an orientation $o(\Lambda)$ of $\Lambda$, we see that $U$ is determined by $(\Lambda, o(\Lambda))$ up to right multiplication by a matrix in $S O(n)$.

Lemma 3.13. Let $u:(D, \partial D) \rightarrow(M, L)$ be a J-holomorphic disk with boundary on a Lagrangian L. Suppose the Maslov index of $u, \mu(u)$, satisfies $\mu(u) \bmod 2=1$. Then $\left.T L\right|_{\partial D}$ is a non-trivial bundle.

Proof. Consider the commutative diagram

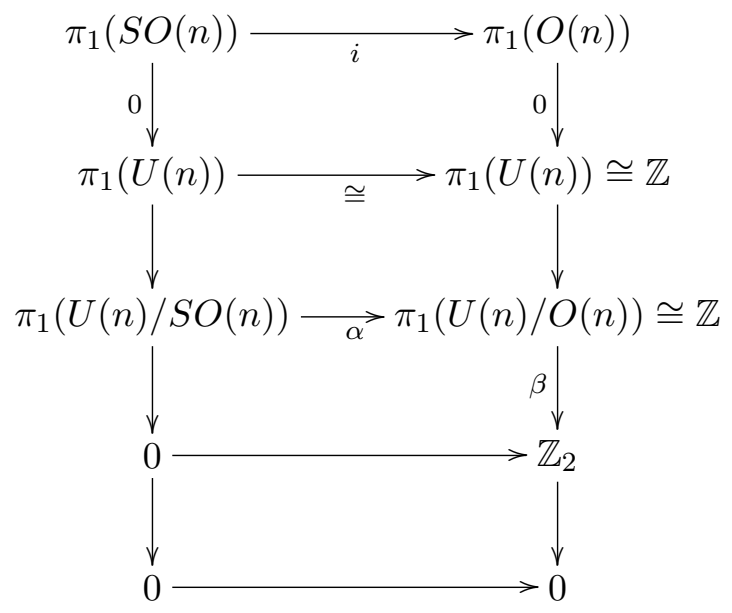

Note that the vertical exact sequences in the diagram are taken from the respective homotopy long exact sequences. Note, too, that it follows from the diagram that the map $\beta$ is onto, and therefore that the map $\alpha$ is multiplication by 2 . Identifying the Maslov class of a loop $\gamma$ of Lagrangians with 
$[\gamma] \in \pi_{1}(U(n) / O(n))$, we see that the Maslov class of any loop $\gamma$ of oriented Lagrangians is even. Now consider a trivialization $\Phi: u^{*} T M \rightarrow D \times \mathbb{C}^{n}$. If $\left.T L\right|_{\partial D}$ is trivial, then the loop of Lagrangians $\left.\Lambda \circ \Phi\right|_{\partial D} \rightarrow U(n) / O(n)$ is a loop of oriented Lagrangians, and therefore $\mu(u)$ is even. This concludes the proof.

Lemma 3.14. Let $(M, \omega, \phi)$ be a four-dimensional real symplectic manifold with real structure $\phi$. Denote the fixed point set of $\phi$ by $L$, and let $E \in H_{2}(M ; \mathbb{Z})$ be a homology class such that $E \cdot E=-1$. Suppose $u:\left(\mathbb{C} P^{1}\right.$, $\sigma, i) \rightarrow(M, \omega, J)$ is a J-holomorphic embedding such that $u_{*}\left[\mathbb{C} P^{1}\right]=E$, and such that the intersection $\operatorname{Im}(u) \cap L \cong S^{1}$. Then the intersection of $T L$ with the normal bundle of $\operatorname{Im}(u)$, i.e., $T L \cap \nu(\operatorname{Im}(u))$, is non-trivial.

Proof. We note that $c_{1}\left(u^{*} T M\right)=2-1=1$, and that the Maslov number of $u=2 c_{1}(E)=2$. Let $u_{1}, u_{2}: D^{2} \rightarrow M$ denote the two disks which make up $u$. We claim that the Maslov index of each disc must be 1 . First, recall that $\mu\left(u_{1}\right)+\mu\left(u_{2}\right)=\mu(u)$ by the properties of the Maslov index. Second, the involution $\phi: M \rightarrow M$ induces a diffeomorphism from $\operatorname{Im}\left(u_{1}\right)$ to $\operatorname{Im}\left(u_{2}\right)$, and $\phi_{*}: T M \rightarrow T M$ is a vector bundle isomorphism from $u_{1}^{*} T M$ to $u_{2}^{*} T M$. Again, the properties of the Maslov index (see Theorem C.3.5 in McDuff and Salamon [22]) imply that $\mu\left(u_{1}\right)=\mu\left(u_{2}\right)$, and this implies that possibilities other than $(1,1)$ for the Maslov indices of the two discs may not occur. It follows that the bundle $T_{S^{1}} L=T S^{1} \oplus \nu_{L}\left(S^{1}\right)$ is non-trivial by Lemma 3.13, where $\nu_{T L}\left(S^{1}\right)$ denotes the part of the normal bundle of $S^{1}$ which lies in $T L$. Since $T S^{1}$ is trivial, then $\nu_{L}\left(S^{1}\right)$ cannot be, and the lemma is proved.

Lemma 3.15. Let $M$ be a four-dimensional real symplectic manifold with real structure $\phi$. Denote the fixed point set of $\phi$ by $L$, and let $E \in H_{2}(M ; \mathbb{Z})$ be a homology class such that $E \cdot E=-1$ and $\phi_{*} E=-E$. Suppose, furthermore, that there exists an embedding of the surface $\Sigma, i: \Sigma \rightarrow M$, with $i_{*}[\Sigma]=E$. Then $E \cdot L=1 \bmod 2$.

Proof. First, we perturb $i$ so that $i(\Sigma) \cap L$ and $i(\Sigma) \cap \phi \circ i(\Sigma)$ are generic. Let $p \in i(\Sigma) \cap \phi \circ i(\Sigma), p \notin L$. Then $\phi(p) \in i(\Sigma) \cap \phi \circ i(\Sigma), \phi(p) \notin L$, and, in particular, $p$ and $\phi(p)$ do not affect the value of either $E \cdot E \bmod 2$ or $E \cdot L \bmod 2$. Suppose now that $E \cdot L=0 \bmod 2$. Then there exists an even number of points in the intersection $i(\Sigma) \cap L$, and, combined with the above, this implies that there is an even number of points in $i(\Sigma) \cap \phi \circ i(\Sigma)$. However, $i_{*}[\Sigma] \cdot \phi_{*} i_{*}[\Sigma]=1 \bmod 2$, which is a contradiction. Therefore $E$. $L=1 \bmod 2$. 
We recall a version of the Riemann Mapping Theorem from [26] (see also [7]).

Theorem 3.16. Let $D$ denote the unit disk in $\mathbb{C}$, let $\Omega$ be a simply connected domain in $\mathbb{C},(\Omega \neq \mathbb{C})$, and assume that the boundary $\partial \Omega$ is locally connected. Then there is a holomorphic isomorphism $f: D \rightarrow \Omega$ that extends to a continuous map from $\bar{D} \rightarrow \bar{\Omega}$. Moreover, if $\partial \Omega$ is a Jordan curve, then $f$ extends to a homeomorphism from $\bar{D}$ to $\bar{\Omega}$.

We now prove Theorem 1.24.

Proof of Theorem 1.24. Let $u: \Sigma \rightarrow M$ be the embedded $J$-holomorphic curve whose image is $C$. By hypothesis, $[C] \cdot[C]=-1$ so Lemma 3.15 implies that $C \cap L \neq \emptyset$. By Corollary 3.11, $C$ intersects $L$ in a circle, whose preimage we denote $S$. Let $D_{1}$ and $D_{2}$ be the two open discs in $C$ with boundary $S$. Note that, for each $x \in D_{1}, \phi(x) \in D_{2}$. Now let $H_{1}$ and $H_{2}$ denote the two hemispheres of $\mathbb{C} P^{1}$ with boundary $\mathbb{R} P^{1}$. By Theorem 3.16 there exists a holomorphic map $\alpha: D_{1} \rightarrow H_{1}$ which extends to a homeomorphism from $\bar{D}_{1}$ to $\bar{H}_{1}$. Now define a map $\tilde{\alpha}: C \rightarrow \mathbb{C} P^{1}$ by

$$
\tilde{\alpha}(x)= \begin{cases}\alpha(x) & \text { if } x \in \bar{D}_{1}, \\ c \circ \alpha(\phi(x)) & \text { if } x \in D_{2},\end{cases}
$$

where $c$ denotes complex conjugation on $\mathbb{C} P^{1}$. We claim that $\tilde{\alpha}$ is holomorphic on all of $\mathbb{C} P^{1}$. First, choose a holomorphic chart $\gamma_{1}: W \subset C \rightarrow \mathbb{C}$ centered at a point $x \in \mathbb{R} P^{1}$ which sends $U \cap \mathbb{R} P^{1}$ to $\mathbb{R}$. Let $\gamma_{2}: V \subset \mathbb{C} P^{1} \rightarrow \mathbb{C}$ be a holomorphic chart centered at $\tilde{\alpha}(x) \in V$, and note that $\tilde{\alpha}$ is holomorphic iff $\gamma_{2} \circ \tilde{\alpha} \circ \gamma_{1}^{-1}$ is holomorphic for any pair of charts. To prove that this is the case, we appeal to Morera's theorem, which we recall below, as stated in Conway [9], following the proof of the Schwartz Reflection Principle.

Theorem 3.17 (Morera's theorem). Let $U$ be a region in $\mathbb{C}$ and let $f: U \rightarrow \mathbb{C}$ be a continuous function such that $\int_{T} f=0$ for every triangular path $T$ in $U$. Then $f$ is analytic in $U$.

To apply this theorem, we need to show that for each triangular path $T \subset U, \int_{T} f=0$. Denote $\gamma_{1}^{-1}(U)$ by $U$, let $U^{+}=U \cap\{z \mid \operatorname{Im}(z)>0\}, U^{0}=$ $\{z \mid \operatorname{Im}(z)=0\}, U^{-}=\{z \mid \operatorname{Im}(z)<0\}$, and $f:=\gamma_{2} \circ \tilde{\alpha} \circ \gamma^{-1}: U \rightarrow \mathbb{C}$. Choose a triangular path $T$ in $U$. We see that $\int_{T} f=0$ iff $\int_{P} f=0$ for any triangular or quadrilateral path $P$ in $U^{+} \cup U^{0}$ and $U^{-} \cup U^{0}$. Furthermore, if $P \subset U^{ \pm}$, then $\int_{P} f=0$, since $f$ is holomorphic on $U^{ \pm}$by definition. We therefore let $T$ 
be the triangle with vertices $[a, b, c]$, where the edge $[b, c]$ is contained in the real axis. The same argument applies for a quadrilateral path. Let $\Delta$ denote the union of the path $T$ and its interior. $f$ is continuous on $U^{+} \cup U^{0}$ by construction, and therefore it is uniformly continuous on $\Delta$. Therefore, for any $\epsilon>0$ there exists a $\delta>0$ such that $\left|z-z^{\prime}\right|<\delta \Longrightarrow\left|f(z)-f\left(z^{\prime}\right)\right|<\epsilon$. Now choose a small $\epsilon>0$, and a $\delta>0$ such that $0<\delta<\epsilon$ and $\left|z-z^{\prime}\right|<$ $\delta \Longrightarrow\left|f(z)-f\left(z^{\prime}\right)\right|<\epsilon$. Pick points $\alpha$ and $\beta$ on the line segments $[a, b]$ and $[a, c]$, respectively, so that $|c-\alpha|<\delta$ and $|b-\beta|<\delta$. Let $T^{\prime}$ and $Q$ be the paths $T^{\prime}=[\alpha, \beta, a, \alpha]$ and $Q=[\alpha, c, b, \beta, \alpha]$ as in Figure 3.1. Then

$$
\int_{T} f=\int_{T^{\prime}} f+\int_{Q} f
$$

However, since $T^{\prime}$ and its interior are contained in $U^{+}, f$ is holomorphic there, and therefore $\int_{T^{\prime}} f=0$.

We now approximate $\int_{Q} f$. First, note that, for $t \in[0,1]$,

$$
|[t \beta+(1-t) \alpha]-[t b+(1-t) c]|<\delta
$$

and therefore

$$
|f(t \beta+(1-t) \alpha)-f(t b+(1-t) c)|<\epsilon .
$$

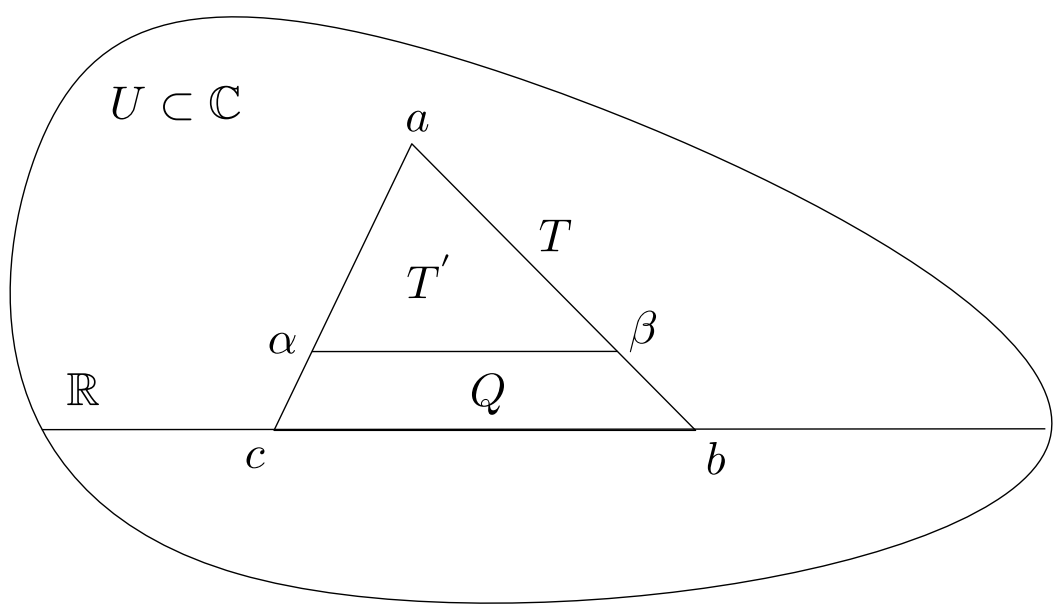

Figure 3.1: Illustration of the paths $T, T^{\prime}$, and $Q$ used in the proof of Theorem 3.16 
Now let $M=\max \{|f(z)| \mid z \in \Delta\}$, and let $l=$ the length of the perimeter of $T$. Then

$$
\begin{aligned}
& \left|\int_{[\alpha, c]} f\right| \leq M|c-\alpha| \leq M \delta \\
& \left|\int_{[\beta, b]} f\right| \leq M|b-\beta| \leq M \delta
\end{aligned}
$$

and

$$
\begin{aligned}
\left|\int_{[b, c]} f+\int_{[\beta, \alpha]} f\right|= & \mid(b-c) \int_{0}^{1} f(t b+(1-t) c) d t \\
& +(\alpha-\beta) \int_{0}^{1} f(t \beta+(1-t) \alpha) d t \mid \\
\leq & |b-c|\left|\int_{0}^{1} f(t b+(1-t) c)-f(t \beta+(1-t) \alpha)\right| \\
& +|(b-c)-(\beta-\alpha)|\left|\int_{0}^{1} f(t \beta+(1-t) \alpha) d t\right| \\
\leq & \epsilon|b-c|+M|(b-\beta)+(c-\alpha)| \\
\leq & \epsilon l+2 M \delta .
\end{aligned}
$$

Therefore,

$$
\left|\int_{T} f\right| \leq \epsilon l+4 M \delta
$$

Since $\epsilon$ is arbitrary, and we may choose $\delta<\epsilon$, it follows that $\int_{T} f=0$, and therefore $f$ is holomorphic. From this we conclude that $\tilde{\alpha}$ is holomorphic as well.

We have now shown the existence of a holomorphic map $\tilde{\alpha}$ that verifies $\phi \circ \tilde{\alpha} \circ c=\tilde{\alpha}$ and $\operatorname{Im}(\tilde{\alpha})=\operatorname{Im}(u)$. Now let $S=C \cap L$.

We now remark that the cohomology class $\left[\tilde{\alpha}^{*} \omega\right] \in H^{2}(\mathcal{L}(0))$ is determined by the integral $\int_{\mathbb{C} P^{1}} \tilde{\alpha}^{*} \omega$, where here we understand $\mathbb{C} P^{1}=\mathcal{L}(0)$. Therefore, for $\lambda^{2}:=\int_{\mathbb{C} P^{1}} \tilde{\alpha}^{*} \omega$, the form $\rho(1, \lambda)$ is in the same cohomology class. By Proposition 2.30, there exists a diffeomorphism $\beta_{0}: \mathcal{L}(0) \rightarrow \mathcal{L}(0)$ such that $\beta_{0}^{*} \tilde{\alpha}^{*} \omega=\rho(1, \lambda)$. Let $\gamma_{0}:=\tilde{\alpha} \circ \beta_{0}$.

Now, by Lemma 3.14 the normal bundle of $S$ in $T L$ is non-trivial. Consider the bundles $\gamma_{0}^{*}(\nu(C))$ and $\nu(\mathcal{L}(0))$, where $\nu(\cdot)$ denotes the normal bundle of the submanifold in question. Since the Chern class of $C$ is 2, the Maslov index of the two disks $D_{1}$ and $D_{2}$ in $C$ with boundary on $L$ is 1 , and 
the restriction of $\nu(C)$ to $L$ is non-trivial, then by Theorem C.3.7 in McDuff and Salamon [22], there is a (complex) isomorphism $\Phi$ between the bundles $\gamma_{0}^{*}\left(\nu\left(D_{1}\right), T L \cap \nu\left(D_{1}\right)\right)$ and $\nu\left(\mathcal{L}(0)^{+}, \mathcal{R}(0)\right)$, where $\mathcal{L}(0)^{ \pm}$denote the upper and lower hemispheres of $\mathcal{L}(0)$, respectively.

Now note that $\tilde{\phi}_{*} \Phi \tilde{c}_{*}$ gives an isomorphism of $\tilde{\alpha}^{*}\left(\nu\left(D_{2}\right), T L \cap \nu\left(D_{2}\right)\right)$ and $\nu\left(\mathcal{L}(0)^{-}, \mathcal{R}(0)\right)$, and therefore the map

$$
\Psi= \begin{cases}\Phi, & (x, v) \in \nu\left(\mathcal{L}(0)^{+}\right) \\ \tilde{\phi}_{*} \Phi \tilde{c}_{*}, & (x, v) \in \nu\left(\mathcal{L}(0)^{-}\right)\end{cases}
$$

is a complex equivariant isomorphism from $\nu(\mathcal{L}(0)) \rightarrow \alpha^{*} \nu(C)$.

Furthermore, since $\Psi$ is a complex bundle isomorphism, it is symplectic as well. It therefore follows from Proposition 2.27, that for some $\delta>0$, we can find a $\mathbb{Z}_{2}$-equivariant map $\beta_{\lambda}: \mathcal{L}(\delta) \rightarrow M$ such that $\beta_{\lambda}^{*} \omega=\rho(1, \lambda)$ which restricts to the symplectomorphism $\gamma_{0}: \mathcal{L}(0) \rightarrow C \subset M$. We may now construct the blow-down by Theorem 1.22 using the equivariant symplectic map $\beta: \mathcal{L}(\delta) \rightarrow M$

\section{Applications to real packing}

We now apply our results to problems of real packing in symplectic four manifolds. That is, given a real, symplectic 4 -manifold $(M, \omega, \phi)$, we wish to know the quantity

$$
p_{L, k}=\sup _{\psi, r} \frac{\operatorname{Vol} \psi\left(\coprod_{i=1}^{k} B_{i}(r)\right)}{\operatorname{Vol} M}
$$

where $\psi: \coprod_{i=1}^{k} B_{i}(r) \hookrightarrow M^{4}$ is a symplectic embedding such that the preimage $\psi^{-1}(\operatorname{Fix}(\phi))=\coprod_{i=1}^{k} B_{i, \mathbb{R}}(r)$. We will, in particular, treat the cases $\left(\mathbb{C} P^{2}, \sigma, \phi\right)$ and $\left(S^{2} \times S^{2}, \sigma_{S^{2}} \oplus \sigma_{S^{2}}, \phi^{\prime}\right)$ with the canonical real structures $\phi, \phi^{\prime}$, where $\mathbb{R} P^{2}=\operatorname{Fix}(\phi)$ and the direct sum of the equators $S^{1} \times S^{1}=$ $\operatorname{Fix}\left(\phi^{\prime}\right)$. In particular, we will prove

Theorem (Theorem 1.26). For the pair $\left(\mathbb{C} P^{2}, \mathbb{R} P^{2}\right)$ with the standard symplectic form and real structure, the real packing numbers $p_{\mathbb{R} P^{2}, k}$ are equal to the absolute packing numbers for $\mathbb{C} P^{2}$.

Theorem 4.1. Let $\phi: S^{2} \rightarrow S^{2}$ be the reflection on $S^{2}$ which sends the upper hemisphere to the lower one and fixes the equator. The real packing numbers for $\left(S^{2} \times S^{2}, \sigma_{S^{2}} \oplus \sigma_{S^{2}}, \phi \oplus \phi\right)$ are equal to the absolute packing numbers for $\left(S^{2} \times S^{2}, \sigma_{S^{2}} \oplus \sigma_{S^{2}}\right)$. 


\begin{tabular}{|c|c|c|c|c|c|c|c|c|c|}
\hline & 1 & 2 & 3 & 4 & 5 & 6 & 7 & 8 & $\geq 9$ \\
\hline$p_{\mathbb{R} P^{2}, k}$ & 1 & $\frac{1}{2}$ & $\frac{3}{4}$ & 1 & $\frac{4}{5}$ & $\frac{24}{25}$ & $\frac{63}{64}$ & $\frac{288}{289}$ & 1 \\
\hline
\end{tabular}

Table 4.2: $p_{S^{1} \times S^{1}, k}\left(S^{2} \times S^{2}, \omega \oplus \omega\right)=p_{k}\left(S^{2} \times S^{2}, \omega \otimes \omega\right)$

\begin{tabular}{lcccccccc}
\hline$k$ & 1 & 2 & 3 & 4 & 5 & 6 & 7 & $\geq 8$ \\
$p_{S^{1} \times S^{1}, k}$ & $\frac{1}{2}$ & 1 & $\frac{2}{3}$ & $\frac{8}{9}$ & $\frac{9}{10}$ & $\frac{48}{49}$ & $\frac{224}{225}$ & 1 \\
\hline
\end{tabular}

The packing numbers for $\left(\mathbb{C} P^{2}, \sigma\right)$ and $\left(S^{2} \times S^{2}, \sigma_{S^{2}} \oplus \sigma_{S^{2}}\right)$ are given in Tables 4.1 and 4.2 above, quoted from [3].

Our basic strategy follows $[3,20]$. We create the blow-up $\tilde{M}$ of $M$ using symplectic and holomorphic embeddings of small balls, and we determine which classes in $H^{2}(M ; \mathbb{R})$ are represented by symplectic forms, in this case trying to increase the area of the exceptional divisors as much as possible.

The following proposition shows that, once we have altered the form on $\tilde{M}$ to increase the area of the exceptional divisors, we are able to show the existence of larger ball embeddings into $M$. It is an adaptation of Proposition 2.1.C in McDuff and Polterovich [20] to real symplectic manifolds.

Proposition 4.2. Let $(M, \omega, \phi)$ be a real symplectic manifold, and let $J$ be an $\omega$-tame almost complex structure which is symmetrically integrable around a set of $k$ points $I=\left\{p_{1}, \ldots, p_{k}\right\} \subset L$, where $L=\operatorname{Fix}(\phi)$. Suppose that for some set of real numbers $\kappa_{q}>0, q \in\{1, \ldots, k\}$, there exists a real symplectic and holomorphic embedding

$$
\psi=\coprod_{q=1}^{k} \psi_{q}: \coprod\left(B\left(1+2 \epsilon_{q}\right), B_{\mathbb{R}}\left(1+2 \epsilon_{q}\right), \kappa_{q}^{2} \omega_{0}, i, c\right) \rightarrow(M, L, \omega, J, \phi)
$$

such that $\psi_{q}(0)=p_{q}$. Let $\Pi: \tilde{M} \rightarrow M$ denote the real symplectic blow-up of $(M, L)$ relative to $\psi$, and let $\tilde{J}, \tilde{\omega}$, and $\tilde{\phi}$ be the complex, symplectic, and real structures, respectively, on $\tilde{M}$ constructed from $J, \omega$, and $\phi$ by blowing-up $M$. Let $C_{q}, q \in\{1, \ldots, k\}$ denote the exceptional curves $\Pi^{-1}\left(\psi_{q}(0)\right)$ added in the blow-up, and let $e_{q} \in H^{2}(M ; \mathbb{Z})$ denote the Poincaré duals of the homology classes $\left[C_{q}\right] \in H_{2}(M ; \mathbb{Z})$. 
Suppose, furthermore, that there exists a smooth family of symplectic forms $\tilde{\omega}_{t}$ on $\tilde{M}$ such that

(1) $\tilde{\omega}_{0}=\tilde{\omega}$ is obtained by a real blow-up relative to the embedding $\psi$,

(2) $\tilde{\omega}_{0}$ tames $\tilde{J}$,

(3) for all $q \in\{1, \ldots, k\},\left.\tilde{\omega}_{t}\right|_{C_{q}}$, the restriction of $\tilde{\omega}_{t}$ to the exceptional divisors $\left\{C_{q}\right\}_{q=1}^{k}$ added in the blow-up, tames $\left.\tilde{J}\right|_{C_{q}}$,

(4) $\phi^{*} \tilde{\omega}_{t}=-\tilde{\omega}_{t}$, so that $\tilde{L}=\Pi^{-1}(L)$ is Lagrangian for each of the forms $\tilde{\omega}_{t}$, and

(5) $\left[\tilde{\omega}_{t}\right]=\left[\Pi^{*} \omega\right]-\sum_{q=1}^{k} \pi \lambda_{q}^{2}(t) e_{q}$ for positive constants $\lambda_{q}(t), 0 \leq t \leq 1$.

Then $(M, L, \omega, \phi)$ admits a real symplectic embedding of $k$ disjoint standard symplectic balls of radii $\lambda_{q}(1), q \in\{1, \ldots, k\}$.

Proof. Since $\tilde{M}$ is the real symplectic and holomorphic blow-up at $k$ real points of $(M, L, J, \phi, \omega)$, then, according to the construction in the proof of Proposition 2.11, there exists a real symplectic and holomorphic embedding

$$
\tilde{\psi}=\coprod_{q=1}^{k} \tilde{\psi}_{q}: \coprod\left(\mathcal{L}\left(1+2 \epsilon_{q}\right), \mathcal{R}\left(1+2 \epsilon_{q}\right), \rho\left(1, \kappa_{q}\right), i, \tilde{c}\right) \rightarrow\left(\tilde{M}, \tilde{L}, \tilde{\omega}_{0}, J, \tilde{\phi}\right)
$$

We will show that for each $q$ there exists a family of equivariant diffeomorphisms $g_{t}: \tilde{M} \rightarrow \tilde{M}, t \in[0,1]$ with the following properties:

(1) $g_{0}=\mathrm{Id}$,

(2) there exists a $\delta \in \mathbb{R}, 0<\delta<1+2 \epsilon$, such that, for all $t, \tilde{\psi}_{q}^{*} g_{t}^{*} \tilde{\omega}_{t}=$ $\rho\left(1, \lambda_{q}(t)\right)$ on $\mathcal{L}(\delta)$,

(3) $g_{t} \circ \tilde{\phi}=\tilde{\phi} \circ g_{t}, g_{t}(\operatorname{Im}(\tilde{\psi}))=\operatorname{Im}(\tilde{\psi})$, and $g_{t}\left(\tilde{\psi}_{q}(\mathcal{L}(0))\right)=\tilde{\psi}_{q}(\mathcal{L}(0))$.

To see this, first note that the $\lambda_{i}(t)$ satisfy the equation

$$
\int_{\mathcal{L}(0)} \tilde{\psi}_{q}^{*} \tilde{\omega}_{t}=\lambda_{i}(t)^{2} \int_{\mathcal{L}(0)} \sigma=\lambda_{i}(t)^{2}
$$

so $\tilde{\psi}_{q}^{*} \tilde{\omega}_{t}$ is in the same cohomology class on $\mathcal{L}(0)$ as $\rho\left(1, \lambda_{q}(t)\right)$. Then, since both of these forms tame $\tilde{i}$ on $\mathcal{L}(0)$, the forms $s \rho\left(1, \lambda_{q}(t)\right)+(1-s) \tilde{\psi}_{q}^{*} \tilde{\omega}_{t}$ are non-degenerate for all $s \in[0,1]$. Therefore, by Proposition 2.30, for each $t$, there exists an equivariant symplectomorphism $F_{q, t}:(\mathcal{L}(0), \rho(1, \lambda(t))) \rightarrow$ $\left(\mathcal{L}(0), \tilde{\psi}^{*} \tilde{\omega}_{t}\right)$ such that $\tilde{c} \circ F_{q, t}=F_{q, t} \circ \tilde{c}$ and $F_{q, t}^{*} \tilde{\psi}^{*} \tilde{\omega}_{t}=\rho\left(1, \lambda_{q}(t)\right)$ on $\mathcal{L}(0)$. 
Since $\tilde{\omega}_{t}$ and $\rho\left(1, \lambda_{q}(t)\right)$ form smooth families of forms, the $F_{q, t}$ must also be smooth with respect to $t$ as well.

We extend the $F_{q, t}$ to an isomorphism of the normal bundle $\nu$ of $\mathcal{L}(0)$ in $\mathcal{L}(1+2 \epsilon)$ by defining $f_{q, t}: \nu \rightarrow \nu$ by $f_{q, t}(z, v)=\left(F_{q, t}(z), v\right)$. Since the restriction of both $\rho(1, \lambda(t))$ and $\rho\left(1, \kappa_{q}\right)=\tilde{\psi}_{q}^{*} \tilde{\omega}$ to the fiber $\nu_{z}$ is $\omega_{0}$, this isomorphism is both equivariant and symplectic. Then, by Theorem 2.27, $F_{q, t}$ extends to an equivariant symplectomorphism $G_{q, t}$ of a neighborhood $\mathcal{N}_{0, t}$ of $\mathcal{L}(0)$ in $(\mathcal{L}(1+2 \epsilon), \rho(1, \lambda(t)))$ to a neighborhood $\mathcal{N}_{1, t}$ of $\mathcal{L}(0)$ in $\left(\mathcal{L}(1+2 \epsilon), \tilde{\psi}^{*} \tilde{\omega}_{t}\right)$. Let $\delta_{q} \in \mathbb{R}, 0<\delta_{q}<1+2 \epsilon$ be such that $\mathcal{L}\left(\delta_{q}\right) \subset \mathcal{N}_{0, t}$ and for all $t \in[0,1]$. Note now that the $\left.G_{q, t}\right|_{\mathcal{L}\left(\delta_{q}\right)}$ also form a smooth family of maps with respect to $t$. Extend $G_{q, t}$ to a smooth family of equivariant differentiable maps from $\mathcal{L}(1+2 \epsilon) \rightarrow \mathcal{L}(1+2 \epsilon)$ which is the identity in a neighborhood of the boundary.

Define $g_{q, t}=\tilde{\psi}_{q} \circ G_{q, t} \circ \tilde{\psi}^{-1}$, extend the $g_{q, t}$ to all of $\tilde{M}$ by the identity outside $\tilde{\psi}\left(\coprod_{q=1}^{k} \mathcal{L}(1+2 \epsilon)\right)$, and denote the extension by $g_{t}$. Then $\tilde{\psi}^{*} g_{t}^{*} \tilde{\omega}_{t}=$ $\rho\left(1, \lambda_{q}(t)\right)$ on $\mathcal{L}\left(\delta_{q}\right)$, making $\tilde{\psi}$ a symplectomorphism with respect to the forms $g_{t}^{*} \tilde{\omega}$ for all $t$.

Now let $\delta=\min \left\{\delta_{q}\right\}_{q=1}^{k}$, and let $\left(M, \omega_{t}\right)$ be the blow-down of $\left(\tilde{M}, g_{t}^{*} \tilde{\omega}_{t}\right)$ using the symplectic and holomorphic embedding $\left.\tilde{\psi}\right|_{\coprod_{q=1}^{k} \mathcal{L}_{q}(\delta)}$. Note that by Theorem 1.22, each form of the family $\omega_{t}$ is cohomologous to $\omega_{0}$. Also, $\omega_{0}$ tames $J$ and $\left[\omega_{0}\right]=[\omega]$, and therefore all the forms $\omega_{t}$ and $s \omega_{0}+(1-s) \omega$, $t, s \in[0,1]$, are symplectic and in the same cohomology class. Furthermore, note that $\frac{d}{d t} \omega_{t}$ is supported on a finite union of balls, and is therefore exact. Therefore, by Proposition 2.30 and Lemma 2.26, there exists a family of equivariant diffeomorphisms $H_{r}: M \rightarrow M, r \in[0,1]$, such that $H_{0}=\mathrm{Id}$ and $H_{1}^{*} \omega=\omega_{1}$. Since $\left(M, \omega_{1}\right)$ admits a real symplectic embedding of $\coprod_{q=1}^{k}(B(1+$ $\left.2 \epsilon), \lambda_{q} \omega_{\text {st }}\right)$, where $\omega_{\text {st }}$ here is the standard symplectic form on $B(1+2 \epsilon)$, this completes the proof.

The following corollary is an easy consequence.

Corollary 4.3. Let $(M, \omega, \phi)$ be a real symplectic manifold with almost complex structure $J$ which tames $\omega$ and is symmetrically integrable around the points $\left\{p_{1}, \ldots, p_{k}\right\}$. Let $\left(\tilde{M}, \tilde{\omega}_{0}, \tilde{\phi}\right)$ be a real manifold obtained by blowing up a real symplectic and holomorphic embedding $\psi$ of balls of radii $\kappa>0, \kappa$ small, and let $\tilde{J}$ be the almost complex structure created in the blow-up.

Now suppose that there exists a real symplectic form $\tilde{\omega}$ on $\tilde{M}$ such that $\tilde{\omega}$ tames the almost complex structure $\tilde{J}$ on $\tilde{M}$ and represents the cohomology 
class

$$
[\tilde{\omega}]=\left[\Pi^{*} \omega\right]-\sum_{i=1}^{k} \pi \lambda_{i}^{2} e_{i}
$$

Then $(M, \omega, \phi)$ admits a real symplectic embedding of $k$ disjoint standard symplectic balls of radii $\lambda_{1}, \ldots, \lambda_{k}$.

Proof. By Proposition 2.11, the blow-up $\tilde{\omega}_{0}$ relative to $\psi$ tames $\tilde{J}$, and therefore the forms $\omega_{s}:=s \tilde{\omega}_{0}+(1-s) \tilde{\omega}$ tame $\tilde{J}$ as well, so the family of forms $\omega_{s}$ satisfies the hypothesis of Proposition 4.2. The conclusion follows.

We now prove a lemma which allows us to symmetrize a symplectic form given a real-structure and a tame, symmetric pseudo-holomorphic structure $J$.

Lemma 4.4. Let $(M, \omega)$ be a symplectic manifold, and let $J$ be an almost complex structure tamed by $\omega$. Suppose there exists an anti-holomorphic involution $\phi$ ( a map $\phi: M \rightarrow M$ such that $\phi^{2}=\operatorname{Id}$ and $\left.\phi_{*} J \phi_{*}=-J\right)$. Then the 2 -form $\bar{\omega}=\frac{1}{2}\left(\omega-\phi^{*} \omega\right)$ has the properties

(1) $\bar{\omega}$ is symplectic,

(2) $\phi^{*} \bar{\omega}=-\bar{\omega}$,

(3) $\bar{\omega}$ tames $J$.

Proof. Since $\omega$ tames $J$, we have that

$$
\bar{\omega}=\frac{1}{2}\left(\omega(v, J v)-\omega\left(\phi_{*} v, \phi_{*} J v\right)\right)=\frac{1}{2}\left(\omega(v, J v)+\omega\left(\phi_{*} v, J \phi_{*} v\right)>0,\right.
$$

and therefore $\bar{\omega}$ tames $J$. It follows that $\bar{\omega}$ is non-degenerate. Furthermore, $\left.d \bar{\omega}=\frac{1}{2} d\left(\omega-\phi^{*} \omega\right)\right)=0$, so $\bar{\omega}$ is closed, and therefore symplectic.

\subsection{Stability of real packing}

We begin with the question of packing stability. Specifically, we show that for a real, rank-1 symplectic 4-manifold of non-Seiberg-Witten simple type, the real packing numbers $p_{\mathbb{R}, k}$ stabilize for large $k$, extending a theorem of Biran[3] to our setting. We begin by recalling several definitions, and we then state our theorem.

Definition 4.5. We say that a symplectic manifold $(M, \omega)$ is of SeibergWitten simple type if the only non-zero Seiberg-Witten invariants are in 
dimension 0. Otherwise, we say that $(M, \omega)$ is of non-Seiberg-Witten simple type. We denote by $\mathcal{C}$ the class of symplectic manifolds which are of nonSeiberg-Witten simple type, and we let $\mathcal{C}_{\mathbb{R}} \subset \mathcal{C}$ denote the real symplectic manifolds in class $\mathcal{C}$. (See Taubes [28] for a definition and overview of the Seiberg-Witten invariants.)

Remark 4.6. As noted in Biran [3], $\mathcal{C}$ contains

(1) symplectic manifolds with $b_{2}^{+}=1$ and $b_{1}=0$, and

(2) ruled symplectic manifolds and their blow-ups.

Definition 4.7. We say that a differential form $\omega$ on $M$ is rank-1 if $[\omega]=$ $c\left[\omega^{\prime}\right]$, where $c \in \mathbb{R}$ and $\left[\omega^{\prime}\right] \in H^{*}(M, \mathbb{Q})$.

Definition 4.8. Let $(M, \omega)$ be a closed symplectic 4-manifold, and let $D_{\omega}$ denote the set

$$
D_{\omega}:=\left\{B \in H_{2}(M ; \mathbb{Z}) \mid \omega(B)>0, c_{1}(B) \geq 2, B \cdot B \geq 0\right\} .
$$

Define $d_{\omega} \in \mathbb{R}$ to be

$$
d_{\omega}:=\inf _{B \in D_{\omega}} \frac{\omega(B)}{c_{1}(B)} \in[0, \infty]
$$

where we adopt the convention that $\inf \emptyset=\infty$.

Theorem 4.9. Let $(M, \omega, \phi)$ be a real symplectic 4-manifold in the class $\mathcal{C}$ where $\omega$ is rank-1 and $\operatorname{Fix}(\phi)=L$. Define $\operatorname{Vol}(M, \omega)=\int_{M} \omega^{2}$, suppose that $0<d_{\omega} \leq \infty$, and let $\lambda_{1}, \ldots, \lambda_{n}<\sqrt{d_{\omega}}$ be positive numbers which satisfy

$$
\sum_{q=1}^{n} \lambda_{q}^{4}<\operatorname{Vol}(M, \omega) .
$$

Then the manifold $(M, \pi \omega, L)$ admits a real symplectic packing by $n$ balls of radii $\lambda_{1}, \ldots, \lambda_{n}$. In particular, if

$$
n \geq \frac{\operatorname{Vol}(M, \omega)}{d_{\omega}^{2}}
$$

then there exists a full real packing of $(M, \pi \omega, L)$ by $n$ equal balls, and $p_{L, n}(M)=p_{n}(M)$, i.e., the relative and absolute packing numbers for $n$ balls are equal. 
As a corollary, we have

Corollary 4.10. The relative packing numbers $p_{\mathbb{R} P^{2}, k}$ for $\left(\mathbb{C} P^{2}, \mathbb{R} P^{2}, \sigma\right)$ are equal to the absolute packing numbers $p_{k}$ for $\left(\mathbb{C} P^{2}, \sigma\right)$ for all $k \geq 9$.

Proof. Note first that $\sigma$ is rank-1, and that $d_{\left(\frac{1}{\pi} \sigma\right)}=\frac{1}{3}$. Therefore, by Theorem 4.9 , there is a full real packing of $\left(\mathbb{C} P^{2}, \mathbb{R}^{2}, \sigma\right)$ for $k \geq 9$.

For the proof of Theorem 4.9, we will appeal to the following result of Biran.

Theorem 4.11 (Biran [3, Theorem 4.1.A]). Let $(M, \omega)$ be a closed symplectic 4-manifold in the class $\mathcal{C}$. Suppose that $0<d_{\omega} \leq \infty$ and let $\lambda_{1}, \ldots$, $\lambda_{n}<\sqrt{d_{\omega}}$ be positive numbers which satisfy

$$
\sum_{q=1}^{N} \lambda_{q}^{4}<\operatorname{Vol}(M, \omega) .
$$

Denote by $\Pi:(\tilde{M}, \tilde{\omega}) \rightarrow(M, \omega)$ a complex blow-up of $(M, \omega)$ at $n$ distinct points. Then the cohomology class

$$
\left[\Pi^{*} \omega\right]-\sum_{q=1}^{N} \lambda_{q}^{2} e_{q} \in H^{2}(\tilde{M} ; \mathbb{R})
$$

admits a symplectic representative $\tilde{\omega}^{\prime}$.

Remark 4.12. If, in addition to satisfying the hypothesis of Theorem 4.11, suppose that $\tilde{\omega}$ is rank-1 and tames an almost complex structure $\tilde{J}$ on $\tilde{M}$. Then, in the proof of Theorem 4.11 given by Biran[3], we may obtain the form $\tilde{\omega}^{\prime}$ by inflating along a single curve using the version of symplectic inflation given in McDuff [19, Lemma 3.1] so that $\tilde{\omega}^{\prime}$ tames $\tilde{J}$ as well. The relevant inflation statement (the analog of Theorem 3.B in Biran [3]) taking into account a tame almost complex structure is

Theorem 4.13. Let $(M, \omega)$ be a symplectic manifold in the class $\mathcal{C}$. Let $A \in H_{2}(M, \mathbb{Q})$ satisfy $\omega(A)>0$ and $A \cdot A>0$, and denote by $\mathcal{E}$ the set of all homology classes which can be represented by $\omega$-symplectic exceptional spheres. Suppose that for every $E \in \mathcal{E}$ we have $A \cdot E \geq 0$, and that $J$ is an $\omega$-tame almost complex structure on $M$. Then there exists a closed 2-form $\rho$ representing the Poincaré dual of $A$, such that for all $y>0, \omega^{\prime}:=\omega+y \rho$ is symplectic and tames $J$. 
We now prove Theorem 4.9 .

Proof of Theorem 4.9. Let $L:=\operatorname{Fix}(\phi)$, and choose an almost complex structure $J$ which is symmetrically integrable around the points $\left\{p_{1}, \ldots, p_{k}\right\} \in L$. By Theorem 1.21, we construct the real blow-up of $(M, \omega)$ with respect to small, real symplectic balls of radius $\epsilon>0$ centered at the points $\left\{p_{i}\right\}_{i=1}^{k}$, and we obtain the real symplectic manifold $\left(\tilde{M}, \tilde{\omega}_{\epsilon}, \tilde{\phi}\right)$ and an almost complex structure $\tilde{J}$ which is tamed by $\tilde{\omega}_{\epsilon}$. By Remark 4.12 , for every $\lambda>0$ such that $\lambda<\sqrt{d_{\omega}}$ and $\sum_{q=1}^{N} \lambda_{q}^{4}<\operatorname{Vol}(M, \omega)$, there exists a symplectic form $\tilde{\omega}_{\lambda}$ which tames $\tilde{J}$ and represents the cohomology class $\left[\Pi^{*} \omega\right]-\sum_{q=1}^{N} \lambda_{q}^{2} e_{q}$. Now note that by Lemma 4.4 , the family of symplectic forms $\tilde{\omega}_{\mathbb{R}, \lambda}=\frac{1}{2}\left(\tilde{\omega}_{\lambda}-\phi^{*} \tilde{\omega}_{\lambda}\right)$ satisfies $\phi^{*} \tilde{\omega}_{\mathbb{R}, \lambda}=-\tilde{\omega}_{\mathbb{R}, \lambda}$, and by Lemma 4.4 these forms also tame $\tilde{J}$. Therefore, by Corollary $4.3,(M, \pi \omega, \phi)$ admits a real symplectic embedding of balls of radius $\lambda$, which proves the theorem.

\subsection{Obstructions to real packing}

We will now show that the real packing numbers below the stable range for real rank-1 symplectic manifolds in the class $\mathcal{C}$ are also identical to the absolute packing numbers.

This follows from a refined version of Theorem 4.9, following Theorem 6.A in Biran [3]. We begin with some definitions.

Definition 4.14. Let $\left(M^{4}, \omega, \phi\right)$ be a real symplectic four-manifold in the class $\mathcal{C}$. Let $\Pi: \tilde{M}_{k} \rightarrow M$ be a real blow-up at $k$ points in $L:=\operatorname{Fix}(\phi)$, and let $\mathcal{E}_{k} \subseteq H_{2}\left(\tilde{M}_{k} ; \mathbb{Z}\right)$ be the subset of homology classes representing symplectic exceptional spheres in $\tilde{M}_{k}$. Let $\Pi_{*}: H_{2}\left(\tilde{M}_{k} ; \mathbb{Z}\right) \rightarrow H_{2}(M ; \mathbb{Z})$ be the projection induced by $\Pi$, and let

$$
\begin{aligned}
\mathcal{E}_{k}{ }^{\prime} & =\Pi_{*}\left(\mathcal{E}_{k}\right) \backslash\{0\} \subset H_{2}(M ; \mathbb{Z}), \\
d^{\prime}{ }_{k} & =\inf _{B \in \mathcal{E}^{\prime}{ }_{k}, c_{1}(B) \neq 1} \frac{\omega(B)}{c_{1}(B)-1} .
\end{aligned}
$$

Remark 4.15. We recall that any exceptional sphere is embedded by definition (see Definition 1.23).

Theorem 4.16. Let $\left(M^{4}, \omega, \phi\right)$ be a real, rank-1 symplectic 4-manifold in the class $\mathcal{C}$. Then

$$
p_{\mathbb{R}, k}=\min \left\{\frac{k d_{k}^{\prime 2}}{2 \operatorname{Vol}(M, \omega)}, 1\right\} .
$$

In particular, the real and absolute packing numbers are equal. 
The proof is an adaptation to our setting of the proof of Theorem 6.A of Biran [3].

We will need the following lemma. We state here the version quoted in Biran [3]. Part 1 follows from Lemma 3.1 in McDuff [17] (see also Proposition 2.3.A in McDuff and Polterovich [20]), and Part 2 follows from the same lemma cited above and the positivity of intersections for $J$-holomorphic curves.

Lemma 4.17. Let $\left(M^{4}, \omega\right)$ be a closed symplectic 4-manifold. Denote by $\mathcal{E}$ the set of all homology classes which can be represented by $\omega$-symplectic exceptional spheres. Then

(1) $\mathcal{E}$ depends only on the deformation class of $\omega$;

(2) if $E^{\prime}, E^{\prime \prime}$ are distinct classes in $\mathcal{E}$, then $E^{\prime} \cdot E^{\prime \prime} \geq 0$.

Proof of Theorem 4.16. Let $L:=\operatorname{Fix}(\phi)$, and use Theorem 1.21 to construct the real blow-up of $M$ with respect to the symplectic embedding of $k$ balls of radius $\epsilon$ centered at points $\left\{p_{1}, \ldots p_{k}\right\}$. Let $\left(\tilde{M}, \tilde{\omega}_{\epsilon}, \tilde{\phi}\right)$ denote the resulting manifold, symplectic form, and real structure, respectively, created in the blow-up, and let $\tilde{J}$ denote the resulting $\tilde{\omega}$-tame almost complex structure on $\tilde{M}$ with $\tilde{\phi}_{*} \tilde{J}=-\tilde{J} \tilde{\phi}_{*}$.

We first show that $d^{\prime}{ }_{k}>0$. Recall that $\mathcal{E}_{k}$ is the set of homology classes in $H_{2}(\tilde{M} ; \mathbb{Z})$ that can be represented by a $\tilde{\omega}_{\epsilon}$-symplectic exceptional sphere, and write $E \in \mathcal{E}_{k}$ as $E=B-\sum_{j=1}^{k} m_{j} E_{j}$, where $E_{j}=\Pi^{-1}\left(p_{j}\right)$ and $B \in$ $H_{2}(M ; \mathbb{Z})$. If $B=0$, then from $E \cdot E=-1$ it follows that $E=E_{j}$ for some $j \in\{1, \ldots, k\}$.

If $B \neq 0$, then $E \neq E_{j}$ for any $j$, so by Lemma 4.17 , part 4.17 , we have that $E \cdot E_{j} \geq 0$, so $m_{j} \geq 0$ for all $1 \leq j \leq k$. Therefore,

$$
\begin{aligned}
\omega(B) & =\tilde{\omega}_{\epsilon}(B)+\sum_{j=1}^{k} \pi \epsilon^{2} e_{j}(B) \\
& =\tilde{\omega}_{\epsilon}(B) \\
& =\tilde{\omega}_{\epsilon}(E)+\sum_{j=1}^{k} m_{j} \tilde{\omega}_{\epsilon}\left(E_{j}\right) \\
& >0,
\end{aligned}
$$

since both $E$ and all the $E_{j}$ are, by definition, represented by embedded $\tilde{\omega}_{\epsilon}$ symplectic spheres. 
Moreover,

$$
1=c_{1}(E)=c_{1}(B)-\sum_{j=1}^{k} m_{j},
$$

so if $B \neq 0$ and at least one $m_{j}>0$, then

$$
c_{1}(B)-1=\sum_{j=1}^{k} m_{j}>0,
$$

and therefore $d^{\prime}{ }_{k}>0$. (Note that if there are no $m_{j}>0$, then $E=B$ and $1=c_{1}(E)=c_{1}(B)$, so $B$ is not considered in the infimum defining $d^{\prime}{ }_{k}$.)

We now remark that the upper bounds on the absolute packing numbers proven in Theorem 6.A of Biran [3] are also upper bounds on the real packing numbers. Therefore

$$
p_{\mathbb{R}, k} \leq \min \left\{\frac{k d_{k}^{\prime 2}}{2 \operatorname{Vol}(M, \omega)}, 1\right\} .
$$

To show that the lower bounds are the same, choose $\lambda>0$ such that

$$
\lambda^{2}<\min \left\{\frac{1}{\pi} d_{k}^{\prime}, \frac{1}{\pi} \sqrt{\frac{2 \operatorname{Vol}(M, \omega)}{k}}\right\},
$$

and consider the cohomology class

$$
a=\left[\Pi^{*} \omega\right]-\pi \lambda^{2} \sum_{j=1}^{k} e_{j},
$$

where the $e_{j}$ are the Poincaré duals of $\Pi^{-1}\left(p_{j}\right)$. Let $A$ be the Poincaré dual of $a$, and assume without loss of generality that $a$ is a rank-1 cohomology class. It is clear that $A \cdot A>0$, and, by taking $\epsilon$ small enough, we have that $\tilde{\omega}_{\epsilon}(A)>0$ as well.

We now claim that, for any $E \in \mathcal{E}_{k}, A \cdot E>0$. To see this, again, write $E=B-\sum_{j=1}^{k} m_{j} E_{j}$. If $B=0$, then, as above, $E=E_{j}$ for some $1 \leq j \leq k$, and therefore $A \cdot E>0$.

Now suppose $B \neq 0$. In this case, $E \neq E_{j} \forall j \in\{1, \ldots, k\}$, and it follows from Lemma 4.17, part 4.17 that $m_{j} \geq 0$ for $1 \leq j \leq k$. If, furthermore, $m_{j}=$ 0 for all $j \in\{1, \ldots, k\}$, then $E=B$. By equation (4.1) we have $\omega(B)>0$, 
and therefore

$$
A \cdot E=\omega(B)-0>0
$$

It only remains to consider the case when at least one $m_{j} \geq 1$. In this case, equation (4.2) gives

$$
\begin{aligned}
A \cdot E & =\omega(B)-\pi \lambda^{2} \sum_{j=1}^{k} m_{j} \\
& =\omega(B)-\pi \lambda^{2}\left(c_{1}(B)-1\right)>0,
\end{aligned}
$$

where the last inequality follows because $c_{1}(B)-1>0$ by equation 4.3, $B \in \mathcal{E}_{k}{ }^{\prime}$ by definition, and $\pi \lambda^{2}<d^{\prime}{ }_{k}$ by hypothesis. This proves the claim.

It now follows from Theorem 4.13 that there exists a closed 2-form $\rho$ representing the class $a=P D(A)$, such that $\forall y>0, \tilde{\omega}_{y}=\frac{1}{y} \tilde{\omega}_{\epsilon}+\rho$ is symplectic and tames $\tilde{J}$. Furthermore, by Lemma 4.4 , we may take each $\tilde{\omega}_{y}$ to satisfy $\phi^{*} \tilde{\omega}_{y}=-\tilde{\omega}_{y}$ as well. By Corollary $4.3,(M, \omega)$ admits a symplectic packing by $k$ equal balls of radius arbitrarily close to $\lambda$. Since this is true for every $\lambda$ that satisfies equation (4.4), we have

$$
\lambda_{\max }^{2} \geq \min \left\{\frac{1}{\pi}{d^{\prime}}_{k}, \frac{1}{\pi} \sqrt{\frac{2 \operatorname{Vol}(M, \omega)}{k}}\right\},
$$

and therefore

$$
p_{\mathbb{R}, k} \geq \min \left\{\frac{k d_{k}^{\prime 2}}{2 \operatorname{Vol}(M, \omega)}, 1\right\}
$$

which completes the proof.

Theorems 1.26 and 4.1 now follow immediately.

Corollary (Theorem 1.26). For the pair $\left(\mathbb{C} P^{2}, \mathbb{R} P^{2}\right)$ with the standard symplectic form and real structure, the real packing numbers $p_{\mathbb{R} P^{2}, k}$ are equal to the absolute packing numbers for $\mathbb{C} P^{2}$.

Corollary (Theorem 4.1). Let $\phi: S^{2} \rightarrow S^{2}$ be the reflection on $S^{2}$ which sends the upper hemisphere to the lower one and fixes the equator. The relative packing numbers for $\left(S^{2} \times S^{2}, \sigma_{S^{2}} \oplus \sigma_{S^{2}}, \phi \oplus \phi\right)$ are equal to the absolute packing numbers for $\left(S^{2} \times S^{2}, \sigma_{S^{2}} \oplus \sigma_{S^{2}}\right)$. 


\section{References}

[1] S. Anjos, F. Lalonde and M. Pinsonnault, The homotopy type of the space of symplectic balls in rational ruled 4-manifolds, Geom. Topol. $\mathbf{1 3}(2)$ (2009), 1177-1227.

[2] J.-F. Barraud and O. Cornea, Lagrangian intersections and the Serre spectral sequence, Ann. Math. (2) 166(3) (2007), 657-722.

[3] P. Biran, Symplectic packing in dimension 4, Geom. Funct. Anal. 7(3) (1997), 420-437.

[4] P. Biran and O. Cornea, Rigidity and uniruling for Lagrangian submanifolds, Geom. Topol. 13(5) (2009), 2881-2989.

[5] G.E. Bredon, Introduction to compact transformation groups, Pure Appl. Math., 46, Academic Press, New York, 1972.

[6] L. Buhovsky, A maximal relative symplectic packing construction, J. Symplectic Geom. 8(1) (2010), 67-72.

[7] R.B. Burckel, An introduction to classical complex analysis, Vol. 1, Pure and Applied Math., 82, Academic Press Inc. [Harcourt Brace Jovanovich Publishers], New York, 1979.

[8] A.C. da Silva, Lectures on symplectic geometry, Lecture Notes in Math., 1764, Springer-Verlag, Berlin, 2001.

[9] J.B. Conway, Functions of one complex variable, 2nd edn., Graduate Texts in Math., 11, Springer-Verlag, New York, 1978.

[10] P. Griffiths and Joseph Harris, Principles of algebraic geometry, Wiley Classics Library, John Wiley \& Sons Inc., New York, 1994, Reprint of the 1978 original.

[11] V. Guillemin and S. Sternberg, Birational equivalence in the symplectic category, Invent. Math. 97(3) (1989), 485-522.

[12] K. Kawakubo, The theory of transformation groups, Japanese edn., Oxford University Press, New York, 1991.

[13] F. Lalonde and D. McDuff, J-curves and the classification of rational and ruled symplectic 4-manifolds, in 'Contact and symplectic geometry (Cambridge, 1994)', 8, 3-42, Cambridge University Press, Cambridge, 1996.

[14] - The classification of ruled symplectic 4-manifolds, Math. Res. Lett. 3(6) (1996), 769-778. 
[15] F. Lalonde and M. Pinsonnault, The topology of the space of symplectic balls in rational 4-manifolds, Duke Math. J. 122(2) (2004), 347-397.

[16] E. Lerman, Symplectic cuts, Math. Res. Lett. 2(3) (1995), 247-258.

[17] D. McDuff, The structure of rational and ruled symplectic 4-manifolds, J. Amer. Math. Soc. 3(3) (1990), 679-712.

[18] - The local behaviour of holomorphic curves in almost complex 4-manifolds, J. Differential Geom. 34(1) (1991), 143-164.

[19] Symplectomorphism groups and almost complex structures, in 'Essays on geometry and related topics', Vol. 1, 2, Enseignement Math., 38, 527-556, Geneva, 2001.

[20] D. McDuff and L. Polterovich, Symplectic packings and algebraic geometry, Invent. Math. 115(3) (1994), 405-434, With an appendix by Yael Karshon.

[21] D. McDuff and D. Salamon, Introduction to symplectic topology, 2nd edn., Oxford Mathematical Monographs, The Clarendon Press Oxford University Press, New York, 1998.

[22] _ J-holomorphic curves and symplectic topology, 2nd edn., American Mathematical Society Colloquium Publications, 52, American Mathematical Society, Providence, RI, 2012.

[23] J.R. Munkres, Topology: a first course, Prentice-Hall, Englewood Cliffs, NJ, 1975.

[24] J.-P. Ortega and T.S. Ratiu, Momentum maps and Hamiltonian reduction, Progress in Math., 222, Birkhauser, Boston, MA, 2004.

[25] M. Pinsonnault, Symplectomorphism groups and embeddings of balls into rational ruled 4-manifolds, Compos. Math. 144(3) (2008), 787810.

[26] Ch. Pommerenke, Boundary behaviour of conformal maps, Grundlehren der Mathematischen Wissenschaften [Fundamental Principles of Mathematical Sciences], 299, Springer-Verlag, Berlin, 1992.

[27] F. Schlenk, Packing symplectic manifolds by hand, J. Symplectic Geom. 3(3) (2005), 313-340.

[28] C.H. Taubes, The Seiberg-Witten and Gromov invariants, Math. Res. Lett. 2(2) (1995), 221-238. 
[29] I. Wieck, Explicit symplectic packings: symplectic tunnelling and new maximal constructions, Ph.D. thesis, Universität Köln, 2008.

Department of Electrical Engineering

Technion - IsRael Institute of Technology

HAIFA 32000

ISRAEL

E-mail address: rieser@tx.technion.ac.il

ReCEIVED 08/25/2011, ACCEPTED 10/09/2013

I would like to thank Octav Cornea and François Lalonde for their constant encouragement and interesting discussions during this project, and the anonymous reviewer for many helpful suggestions which greatly improved both the content and presentation of this paper. This work was supported in part by the Israel Science Foundation grant $723 / 10$. 
